A Comparison of Large and Small Tropical Cyclones

by

Robert Travis Merrill

P.I. William M. Gray

Department of Atmospheric Science

Colorado State University

Fort Collins, Colorado

DOA NA8IRAD00005

DOA NA8IRH00001

NSF ATM-7923591

Department of

Atmospheric Science

Paper No. 352 


\section{A COMPARISON OF LARE AND SMALL TROPICAL CYCLONES}

By

Robert Travis 䟢erri11

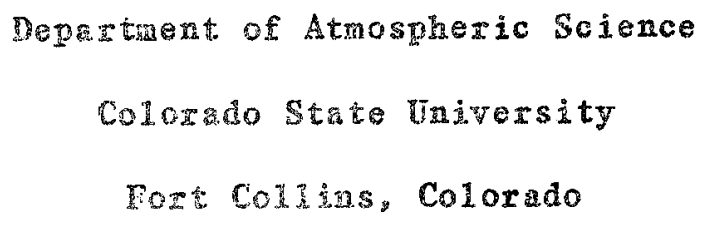

July, 1982 
The structure of the wind field of a tropical cyclone can be roughly described by three measurements: intensity (maximum wind), size (extent of the vortez). and stengtio (average wind speed of the vortex). This paper axmines sta chatology. structure and possible physical processes of tropical eyolones of diverent sizes. Records of tropical cyclone sizes, as indicatod by the radius of the outer closed isobar (ROCI) are described for the period 1961-1969 in the western North Pacific and 1957-1977 in the Atlantic. The climatology of cyclone sizes confirms that typhoons ax charactoristically twice as large as hurricanes, and also reveals brat the typlcal size of cyclones varies seasonally and regionally whth oeean basins and is only weakly correlated with cyclone ansensisy (mazimum wind or minimum pressure). Rawinsonde composites of 3 argo snd small troplad cyclones show that large cyclones have much nore relative angular momentun (RAM) than small ones, while the differences in RAM between intense and weaker cyclones of equivalent size are much less. Some of the implications of this observation are iscussed. and a jypothesis that cyclones grow as a result of an increased convergonce of angurar momentum forced by their environment is prosented. 
TABLE OF CONTENTS

Page

1. INTRODUCLION ......................... 1

1.1 Definitions ................... 3

2. DESCRIPTION OF DATA .................. 6

2.1 Rawinsonde Data .................. 6

2.2 Tropical Cyclone Position and Size Records........ 8

3. CLIMATOLOGY OF TROPICAL CYCEOHE SIZE . . . . . . . . . 11

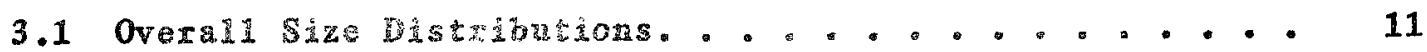

3.2 Seasonal Variability of Tropical Cyclone Size . . . . . 13

3.3 Spatial Variability of Tropical Cyclone Size....... 15

3.4 The Relationship Between Size and Intensity . . . . . . 26

3.5 Characteristio Ganges in Tropical Cyclone Size
and Intensity. . . . . . . . . . . 26

4. STRUCTURE OF IARGE AND SMALL TROPICAL CXCLONES . . . . . . 34

4.1 Introduction. ................... 34

4.2 Mean Structural Differences Between Large and Small

Tropical Cyclones,................ 36

4.3 Statistical Significance of Tangential Wind Differences - 43

4.4 Extent of $15 \mathrm{~ms}(30 \mathrm{kt})$ Surface Winds........ 46

5. MAINTENANCE OE LARGE AND SUALL CYCLONES AND ANGULAR

MOMENTUM CONSTDERATIONS ................. 51

5.1 Introdncton. . . . . . . . . ........ 51

5.2 A Diseussion of Angular Momentum in Tropical Cyclones . . 51

5.3 Observed Angular Monentum Associated With Large and

Smali Tropical Cyclones............... 53

5.4 Implications of Anguger Momentum Constxaints for Cyclone

Size and Growh............... 58

6. SUMMARY AND CONCLUSTONS. .................. 64

ACKNOWLEDGEMENTS. .................... 67

RFFERENCES. ............................ 68

APPENDIX. . . . . . . . . . . . . . . . . . . 71 


\section{INTRODUCTION}

Tropical cyclones have been the subject of extensive study for many years - foreasters have charted their tracks and described their behavior in detail, and sclentists have complled observations of these storms and performed numerical experiments in an effort to unlock their physics. These efforts have met with 1 imited success - we know more about tropical cyclones than we did 25 years ago, but some aspects of tropical cyclone meteorology have gone nearly untouched. In particular, the structure of the hurxicane vortex, in terms of its intensity, strength and size, has yet to be fully described.

The term 'tropical cyclone' implies a neat classification. Yet within this classification we find storms as diverse as cyclone 'Tracy', which struck Darwin, Australia in 1974, and Supertyphoon 'Tip', which occurred in the northwest Pacific in 1979. Tracy had gale force winds $\left(17 \mathrm{~m} \mathrm{~s}^{-1}\right.$ ) over an area only $100 \mathrm{~km}$ across, though its peak winds of 65 $\mathrm{m} \mathrm{s}^{-1}$ made it a severe hurricane (Australian Bureau of Meteorology, 1977). Tip, on the other hand, spread gale force winds over a $2200 \mathrm{~km}$ diametex, an area hundreds of times larger than Tracy (Dunnavan and Diercks, 1980). Figure 1 shows two examples from the Atlantic basin. Camille had maximum winds of $85 \mathrm{~m} \mathrm{~s}^{-1}$, while Faith, though much 1arger, had only $50 \mathrm{~m} \mathrm{~s}^{-1}$ winds. Even within a single season, tropical cyclones come in all sizes just as they move in a variety of ways and attain a variety of intensities. 

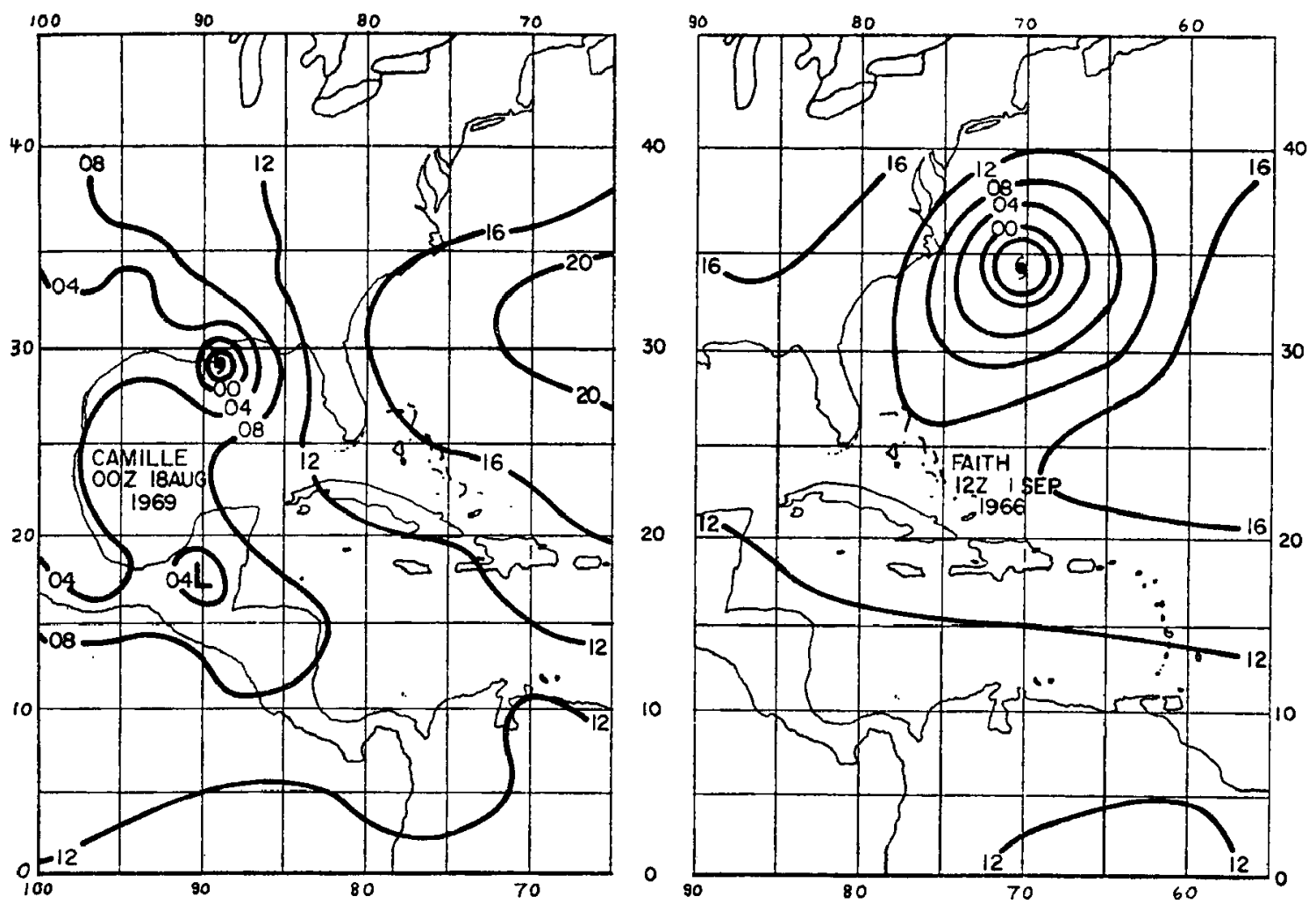

Fig. 1. Surface pressure ana1yses for Camille, a sma11, intense hurricane (left) and Faith, a larger but weaker one (right).

The issue of cyclone size is not unimportant, either. A sma11, intense hurricane or typhoon is easily avoided by shipping, while a larger, yet weaker one, can produce heavy seas over a large area and greatly hamper sea traffic. Other practical concerns come to mind: do large hurricanes break up over land less rapid1y, carrying damaging winds inland far enough to reach populated areas which would otherwise go untonched? Will a large cyclone produce more tornadoes or torrential rains than a smallex one? One of the purposes of the research being presented in this paper is to make these and other investigations possible at a later date by building a data set and climatology of cyclone size. These are described in Chapters 2 and 3 respectively. 
Equaly important are the implications cyclone size has on cyclone dynamics. Large cyclones spread relatively high winds over a larger area and are thus exposed to greater surface losses of angular momentum and kinetic energy, but also gain more moisture through evaporation. The physical influences these processes have on the intensity of the cyclone are not known - nor is it clear how large and small oyclones might interact with their environment. Chapter 4 describes the structure of large and small cyclones and Chapter 5 discusses how these structural differences might affect the dynamics of the cyclone.

\subsection{Definitions}

The topic of cyclone size is a relatively new one, and some confusion has arisen in the past over the terms 'size', 'strength', 'intensity', 'growth', etc. A major point of this paper is that changes in the structure of a tropical oyclone vortex can occur in several different modes. These modes aro illustrated in Fig. 2 and describod below. A summary is given in Table 1.

Intensity. A measure of the extreme value of a meteorological variable in a tropical cyclone. Minimum sea level pressure (MSLP) and maximum sustained winds are usually used to describe the intensity of a cyclone. Intensification refers to a decrease in MSLP or an increase in maximum winds.

Size. The areal extent of a tropical cyclone circulation. It is operationally measured as the extent of winds above a certain speed, usually gale force $\left(17 \mathrm{~m} \mathrm{~s}^{-1}\right)$, or as the average radius of outer closed isobar (ROCI). The latter is used throughout this paper. Growth refers to an expansion of the tropical cyclone circulation. 
Strength. The average wind speed in the cyclone circulation. Note from Fig. 2 that the strength of a cyclone can change even though the intensity (maximum wind) and size (extent of gales) remain constant. Strength is not dealt with explicitly in this paper. 


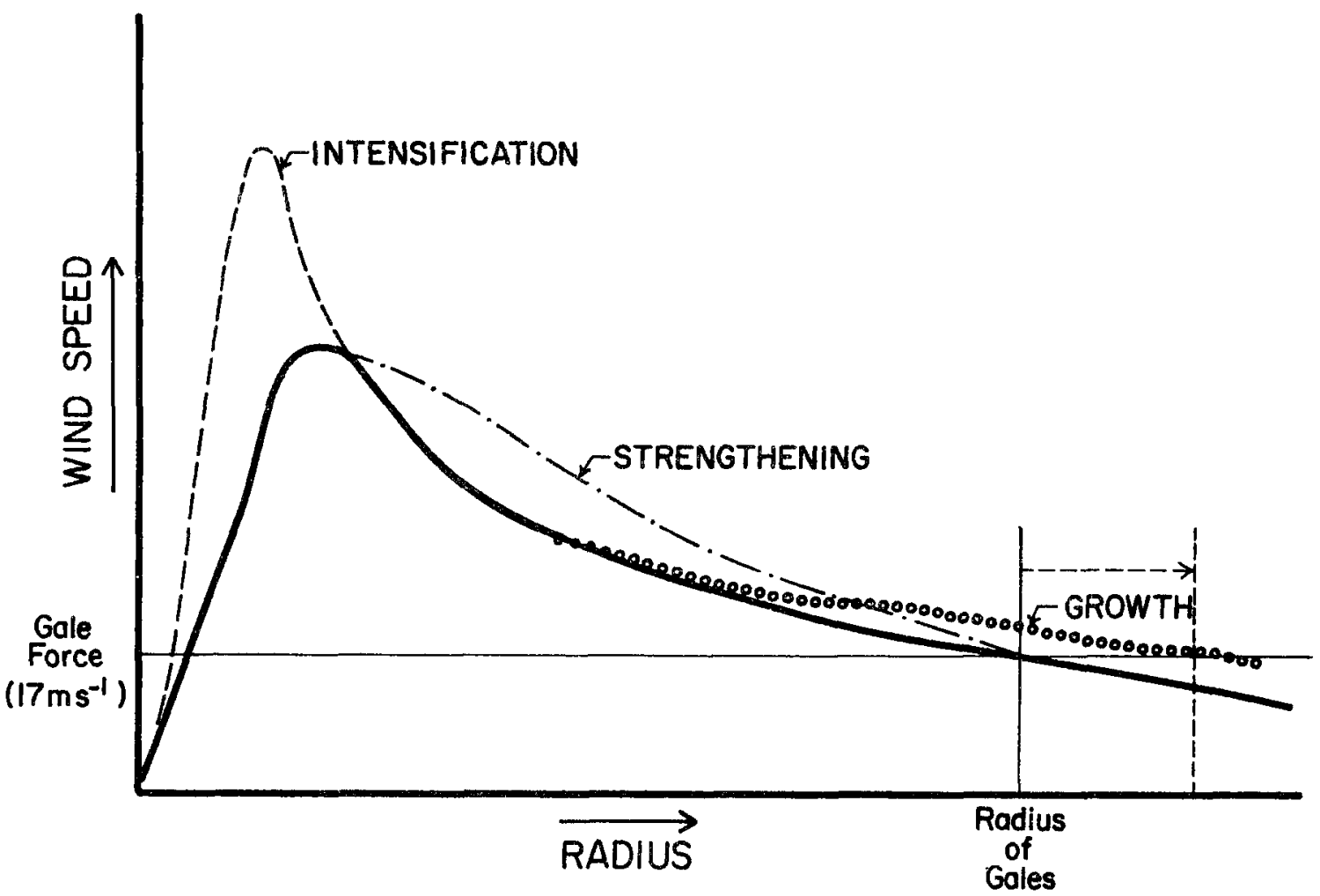

Fig. 2. Schematic of the changes in lower tropospheric tangential wind associated with intensification, strengthening, and growth of a tropical cyclone.

TABLE 1

Definitions of the structure of the tropical cyclone wind field.

Description

Definition

Increase with Time

$\begin{array}{lll}\text { Intensity } & \begin{array}{l}\text { Maximum wind or } \\ \text { Minimum Sea Level Pressure }\end{array} & \text { Intensification } \\ \text { Strength } & \begin{array}{l}\text { Average Wind Speed } \\ \text { Within the Circulation }\end{array} & \text { Strengthening } \\ \text { Size } & \begin{array}{l}\text { Extent of the Cyclone } \\ \text { Circulation (radius } \\ \text { of Gales or of } \\ \text { Outer Closed Isobar) }\end{array} & \text { Growth } \\ & \end{array}$




\section{DESCRIPTION OF DATA}

Four data sets are used in this paper: 1) A set of cyclone

positions and sizes for the Atlantic basin, 1957-1977, 2) positions and 8 izes for the western North Pacific basin, 1961-1969, 3) Atlantic basin rawinsonde observations (Fig. 3) for 1957-1977, and 4) Pacific rawinsonde observations (Fig. 4) for 1961-1969. The tropical cyclone size climatology presented in Chapter 3 is based on the position and size records, and the structure of large and small cyclones as described in Chapter 4 is determined by compositing the rawinsonde observations. The rawinsonde data sets and the compositing approach have been in existence for several years and have been widely used by other members of Professor Gray's tropical cyclone research project. The size records are much less we11 documented, and did not oven exist for the Atlantic prior to this study. Both the rawinsonde data and the track/size data are described in greater detail below.

\subsection{Rawinsonde Data}

Two rawinsonde data sets were used to examine the structure of large and small cyclones - an Atlantic set consisting of about 50,000 soundings from 103 stations over a period of 21 years from 1957-1977, and a Pacific set, with about 18,000 soundings from 28 stations over a 9 year period from 1961-1969. The location of the rawinsonde stations for the Atlantic and Pacific sets are shown in Figs. 3 and 4 respectively. The Pacific set is a subgroup of the 10 year (1961-1970) rawinsonde set used by Frank (1977) and George and Gray (1977) in studies of typhoon 


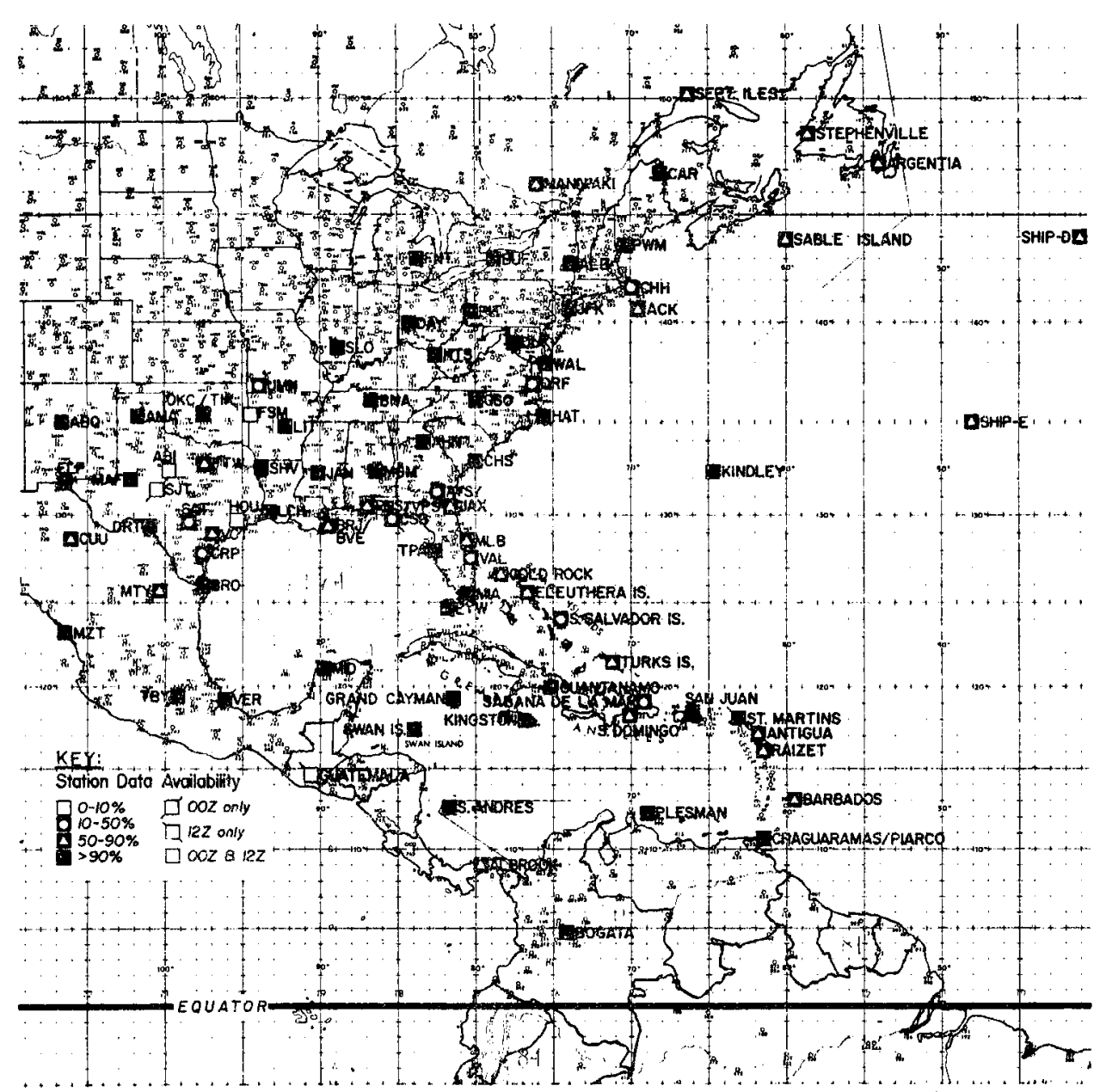

Fig. 3. Location of Atlantic rawinsonde observations making up the rawinsonde composites.

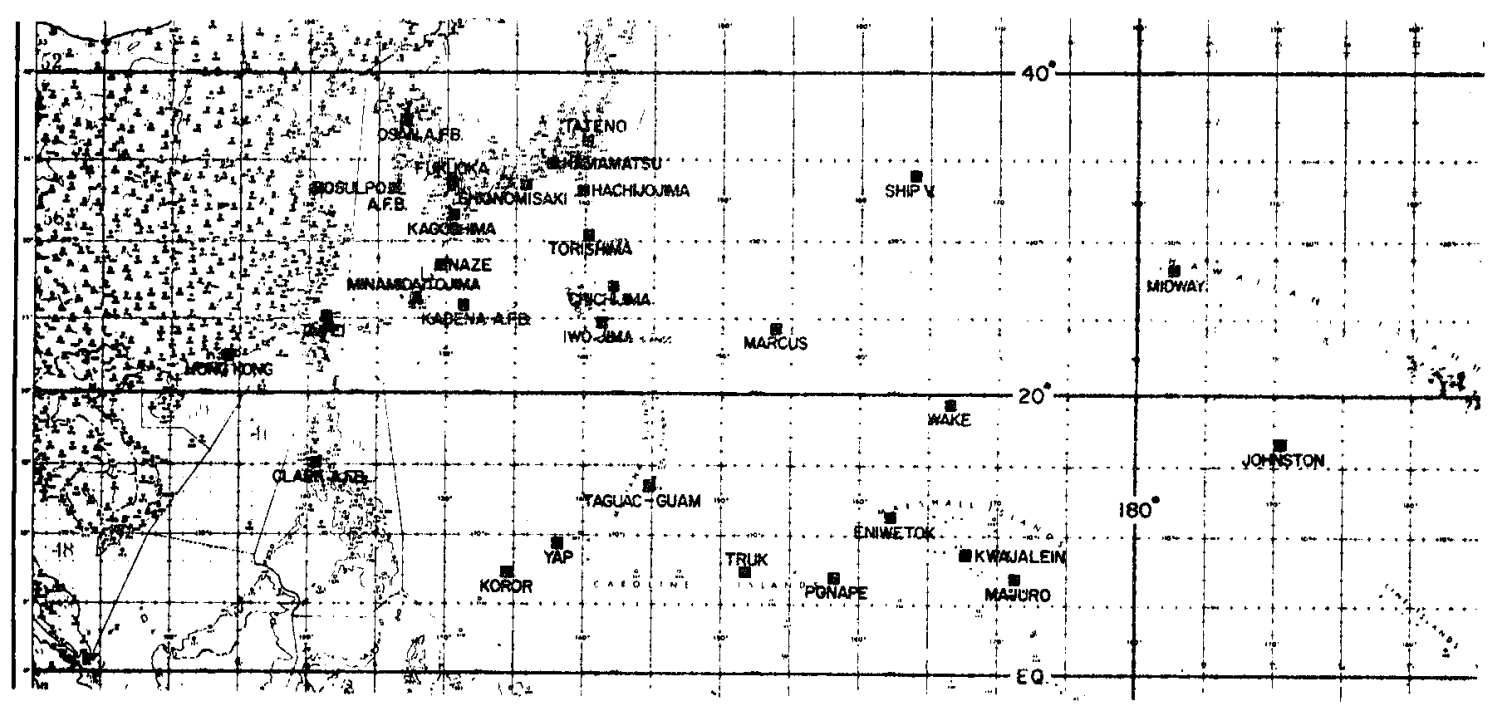

Fig. 4. Location of Pacific rawinsonde observations making up the rawinsonde composites. 
structure, energetics and motion. The Atlantic set is an extension of the 14 year (1961-1974) set used in structure and motion studies of Atlantic tropical weather systems by Chan et a1. (1980), Frank and Gray (1980) and McBride and Zehr (1981). The contents of these sets, the details of their preparation, and the rawinsonde compositing method are described in Gray et al. (1982).

\subsection{Tropical Cyclone Position and Size Records}

The Pacific cyclone position and size data were prepared at the Joint Typhoon Warning Center on Guam and made available by the Naval Environmental Prodiction Research Facility (NEPRF) at Monterey, CA. Each record consists of a position, maximum wind, minimum sea level pressure (MSLP), speed and direction of motion, and average radius of outer closed isobar (ROCI) at 00 GMT and 12 GMT for tropical storms and typhoons during 1961-1969. The set includes 201 cyclones and a total of 2576 records.

The Atlantic basin position set was built using the National Hurricane Center (NHC) track file (Jarvinen and Caso, 1978) which consists of position, estimated maximum sustained winds, and MSLP (where available) at 6 hour intervals. These records were used to compute speed and direction at 00 GMT and 12 GMT, and then merged with ROCI data (determined as described below) to obtain complete records, analogous to those for the Pacific, for the tropical storms and hurricanes of 19571977. The Atlantic position set contains 183 cyclones and 1722 position records.

Measurements of the radius of outer closed isobar are not recorded operationaly in the Atlantic, and therefore had to be extracted from archived NHC surface analyses, available on microfilm at Colorado State 
University. The ROCI is defined as the average of the distances to the north, east, south and west of the cyclone center to the closed isobar having the highest value. These values axe expressed in whole degreos latitude so as to be compatible with existing data for Pacific cyclonos. Prior to 1964, charts were analyzed at $3 \mathrm{mb}$ intervals and had to be redrawn at 2 mb intervals for consistency with Pacific and 1ater Atlantic analyses, and some additional reanalysis of charts was performed when needed. Occasionally, particularly when a cyclone was just forming, the outer clased isobar was elongated or distorted. In such cases, the next-lower valued isobar was taken as the outer closed isobar. A schematic of the measurement technique is shown in Fig. 5 . The ROCI has its disadvantages as a measure of cyclone size. It is somewhat dependent on the environmental pressure gradient, is not as physically relevant as is the wind field, and is subjectively determined. It is nevertheless the best measure of cyclone size that is available throughout the life of a cyclone. Because the surface pressure is generally smooth and we11-behaved, it is an easier field to estimate than is wind, which may vary sharply in the horizontal. Since a size estimate is needed for every cyclone position on file so that a climatology can be made, the ROCI is believed to be the best choice of a size estimate. In addition, the relationship between radius of outer closed isobar and the cyclone wind field is consistent and statistically sound. Evidence of this will be provided in Chapter 4. 


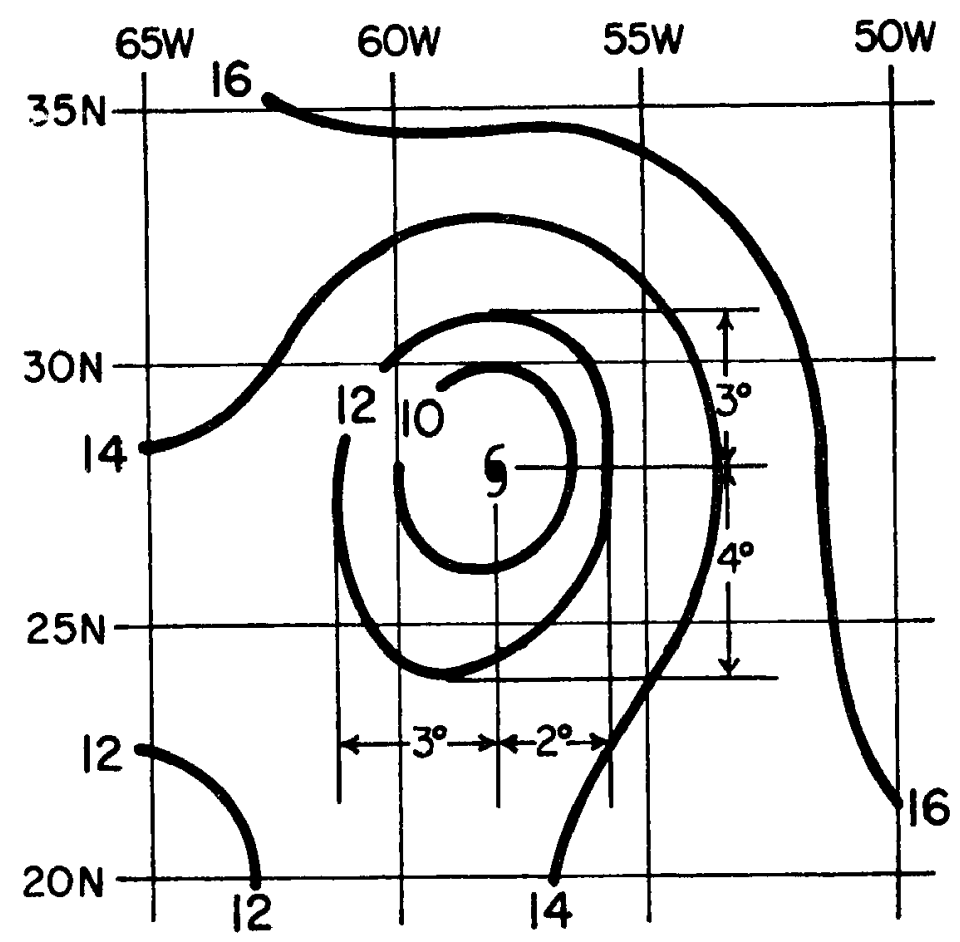

Average $\mathrm{ROCl}=3^{\circ}$ Lat

Fig. 5. Example of the method used to determine the average radius of outer closed isobar (ROCI) of a tropical cyclone. 


\section{CLIMATOLOGY OF TROPICAL CYCLONE SIZE}

A meteorological phenomenon can of ten be partly understood simply by identifying where, when and with what frequency it occurs. A major component of this paper is a climatology of the tropical cyclone based on size. Such studies are few and 1 imited in scope in the Pacific (Brand, 1972, and Arakawa, 1950) and altogether nonexistent in the At1antic. The climatology will be developed in parallel for each basin. Four topics will be discussed in turn: 1) the overall distribution of cyclone sizes in each ocean basin, 2) the monthly variation of the size distribution, 3) the spatial variation in cyclone size across the basin, and 4) the typical changes in size during the life cycle of a typhoon or hurricane.

\subsection{Overa11 Size Distributions}

The distributions of size of all cyclones for the Atlantic (19571977) and Pacific (1961-1969) are shown in Figs. 6 and 7 respective1y. The size difference between Atlantic and Pacific cyclones which has been previous1y described qualitatively (Atkinson, 1971) is p1ainly evident. Table 2 shows some of the statistical attributes of these distributions. It can be seen that the ROCI of the 'mean' Pacific cyclone is about 1.5 degrees larger than that of the Atlantic cyclone. This represents a difference of about 40 percent in the mean radius between the two basins. For a circular outer closed isobar, the enclosed area is over twice as great for the Pacific cyclone. 


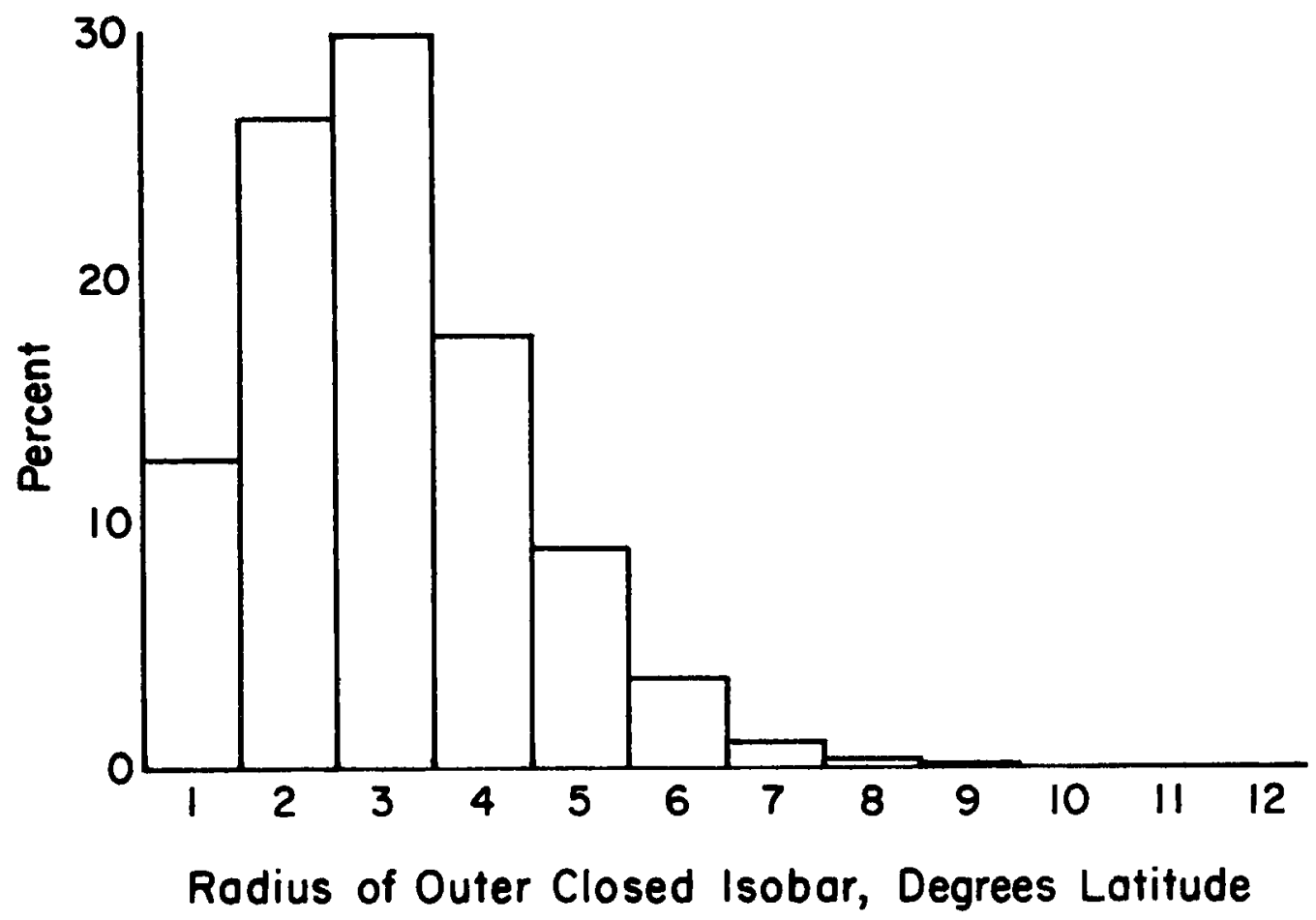

Fig. 6. Percent frequency distribution of sizes of Atlantic tropical cyclones, 1957-1977.

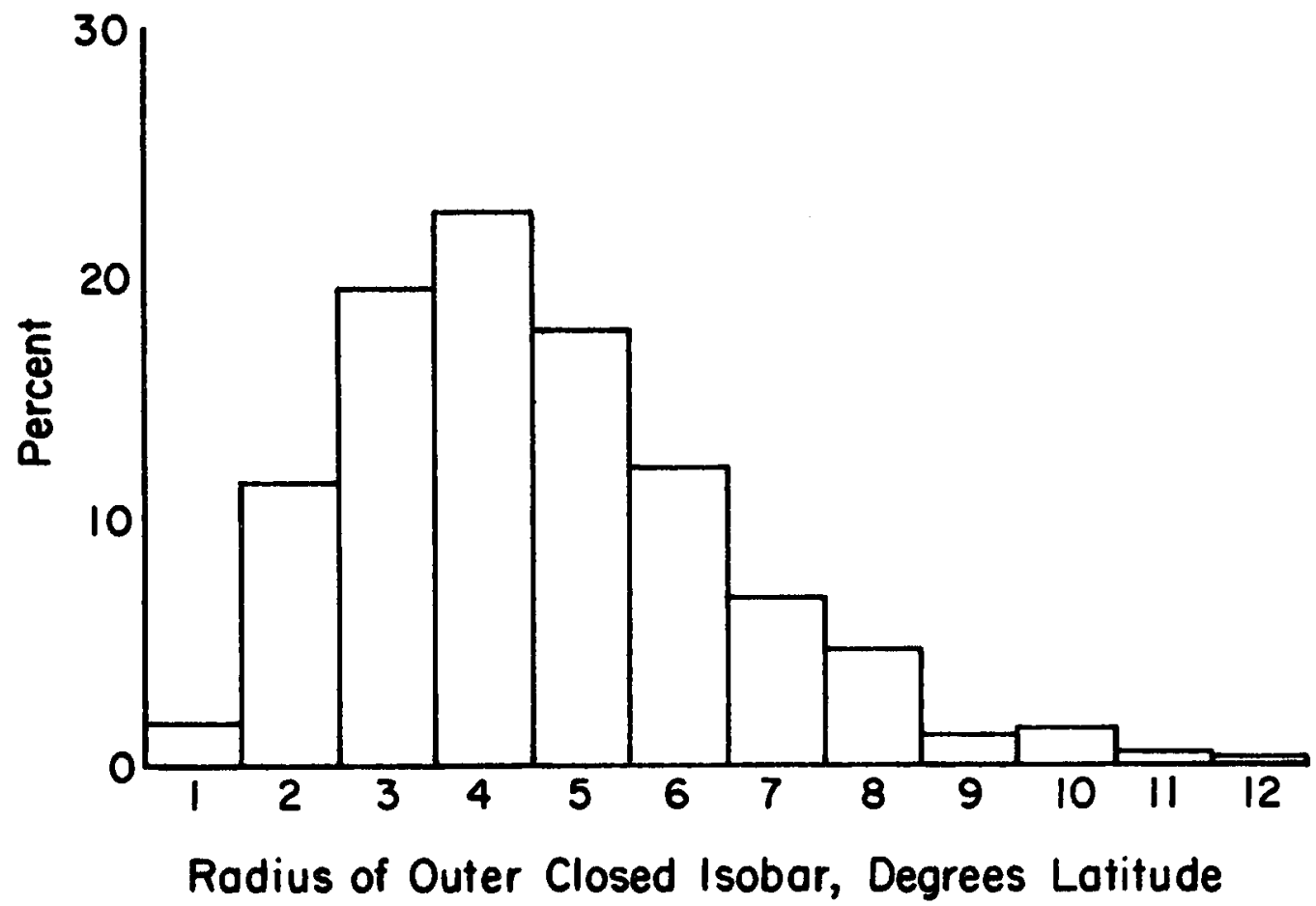

Fig. 7. Same as Fig. 6 but for the Pacific tropical cyclones, 19611969. 
TABLE 2

Statistical attributes of the distributions of tropical cyclone size for the north Atlantic and northwest Pacific basins.

\section{Atlantic $\quad$ Pacific}

Number of Records

1722

2576

RADIUS(degrees latitude)

Mean

3.0

4.6

Standard Deviation

1.4

2.0

Median

2.4

3.8

25 th percentile

1.5

2.6

75 th percentile

3.4

5.2

AREA (degrees latitude) $)^{2}$

Mean

10.9

24.5

Standard Deviation

9.8

21.8

Median

5.9

14.4

25th percentile

2.4

7.0

75 th percentile

11.5

26.1

An immediate inference is that some environmental influence modulates cyclone size and causes a characteristically larger cyclone in the Pacific. If this is the case, it might be expected that this onvironmental control also varies seasonally. The next section examinos this possibility.

\subsection{Seasonal Variability of Tropical Cyclone Size}

The seasonal change of cyclone size is summarized in Fig. 8 (At1antic) and Fig. 9 (Pacific). With the exception of the relatively few Pacific cyclones which occur in the period December-April, the two curves are similar; both display a relative size minimum in mid-summer and a maximum in October. May cyclones are quite rare in the Atlantic, so that value is not too reliable. 


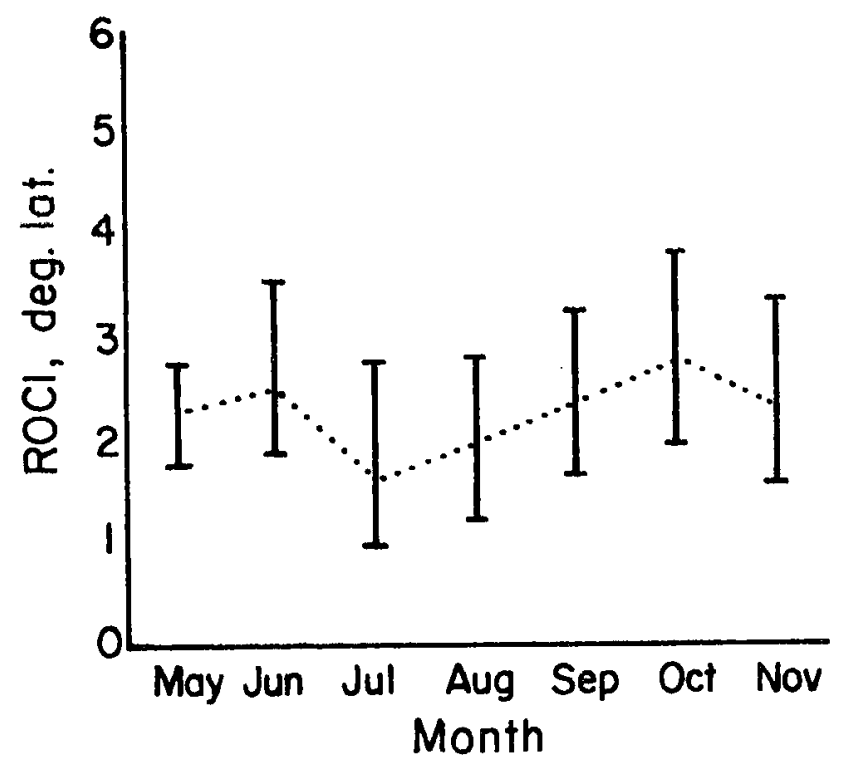

Fig. 8. Monthly progression of median, 25 th, and 75 th percentile of size for Atlantic tropical cyclones (includes all tropical storms and hurricanes), 1957-1977.

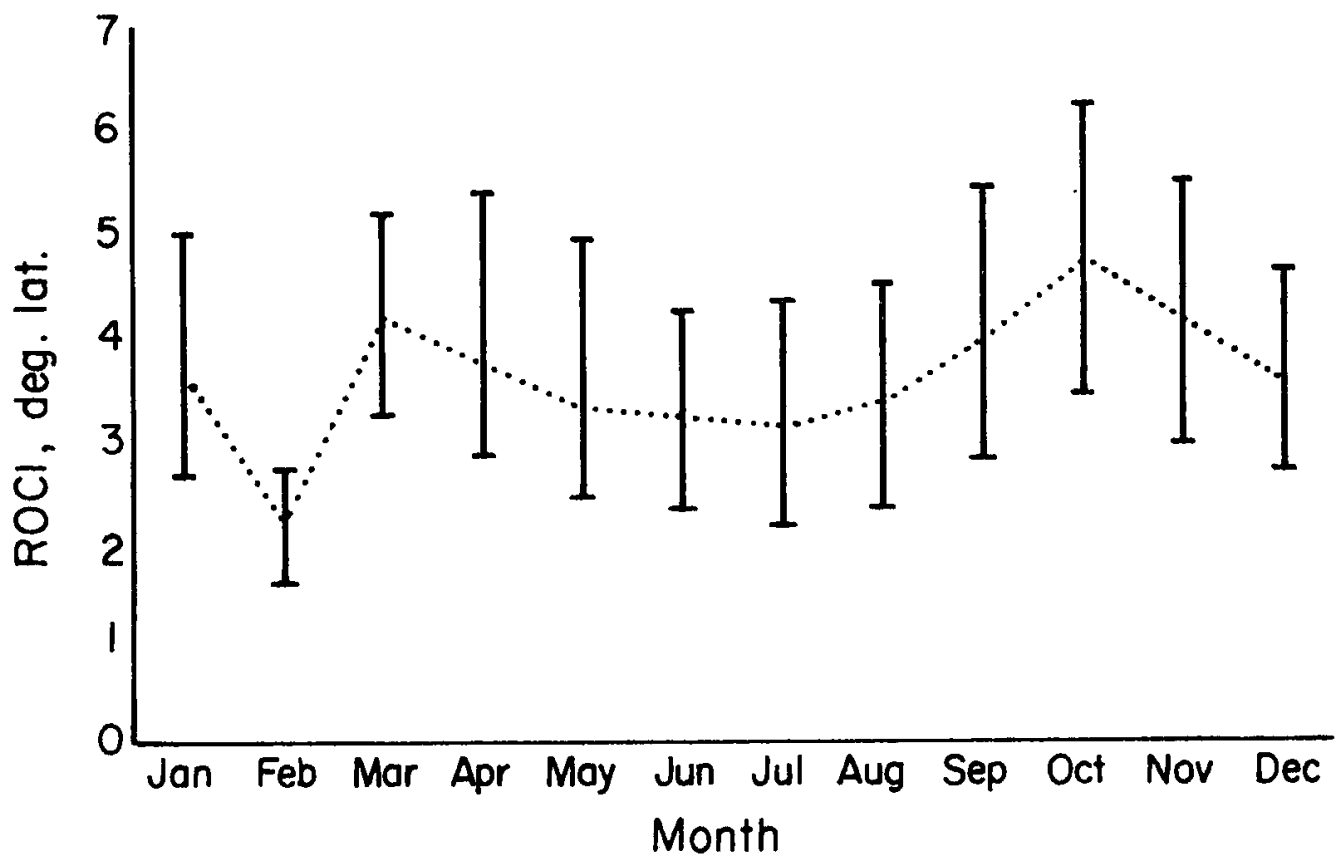

Fig. 9. Same as Fig. 8 but for the Pacific tropical cyclones (includes all tropical storms and typhoons), 1961-1969. 
The simplest explanation for the seasonal variation would be to relate cyclone size and intensity, as defined by MSLP or maximum winds. The strongest cyclones would then be expected in October, with weaker ones in midsummer. Examination of the wind data show this not to be the case, however. Time series of maximum wind of tropical cyclones are presented for the Atlantic in Fig. 10 and Pacific in Fig. 11. In both basins, September cyclones are at least as intense as 0ctober ones and, although a weak intensity minimum does appear in July in the Pacific, the Atlantic shows only a monotonic increase in strength through the midsumer size minimum. Another discrepancy between intensity and size occurs in the spring in the Pacific, where the size maximum in March leads the April intensity maximum. The opposite occurs in fall in both basins; maximum intensity (September) leads maximum size (October). Although trends in intensity account for part of the seasonal trend in size, additional factors appear to be involved.

\subsection{Spatial Variability of Tropical Cyclone Size}

It has been shown (Dunn and Miller, 1960) that cyclone genesis occurs in different parts of the basin at different times of the year. Love (1982) has furnished evidence that periods of tropical cyclone genesis in the northwest Pacific are preceded by synoptic and planetary scale changes in the tropospheric circulation of both hemispheres. In the Atlantic, the few June cyclones that do occur usually form in the northwest Caribbean Sea and the Gulf of Mexico. The area of genesis shifts to the central tropical Atlantic in Angust and September and back to the Caribbean-Gulf area in October in response to environmental controls. Pacific cyclone genesis tends to follow the monsoon trough and genesis occurs at highest 1atitude in Angust (Gray, 1979 and Ding 


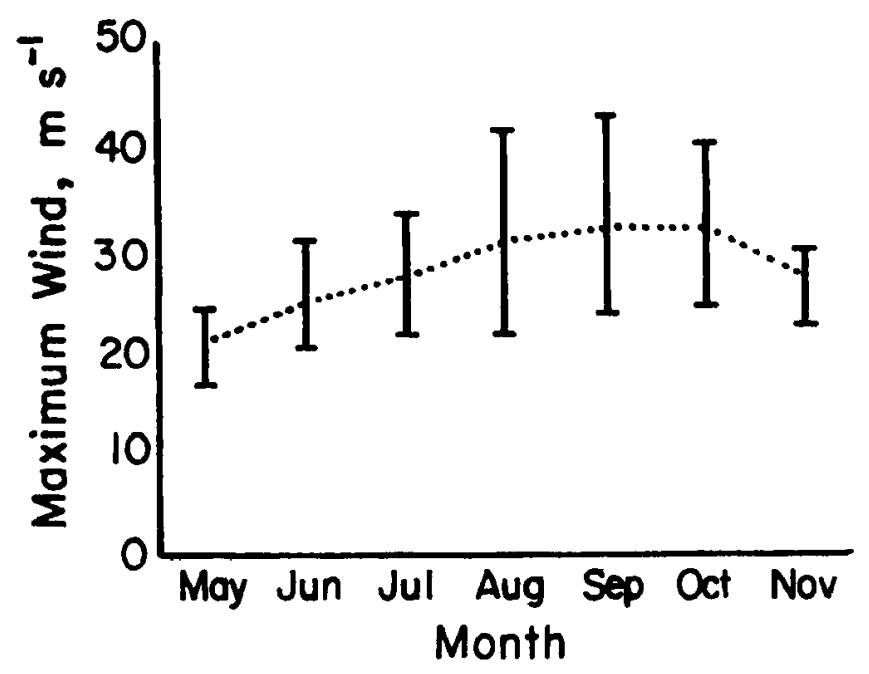

Fig. 10. Monthly progression of median, $25 \mathrm{th}$, and $75 \mathrm{th}$ percentile values of maximum sustained wind of Atlantic tropical cyclones, 19571977.

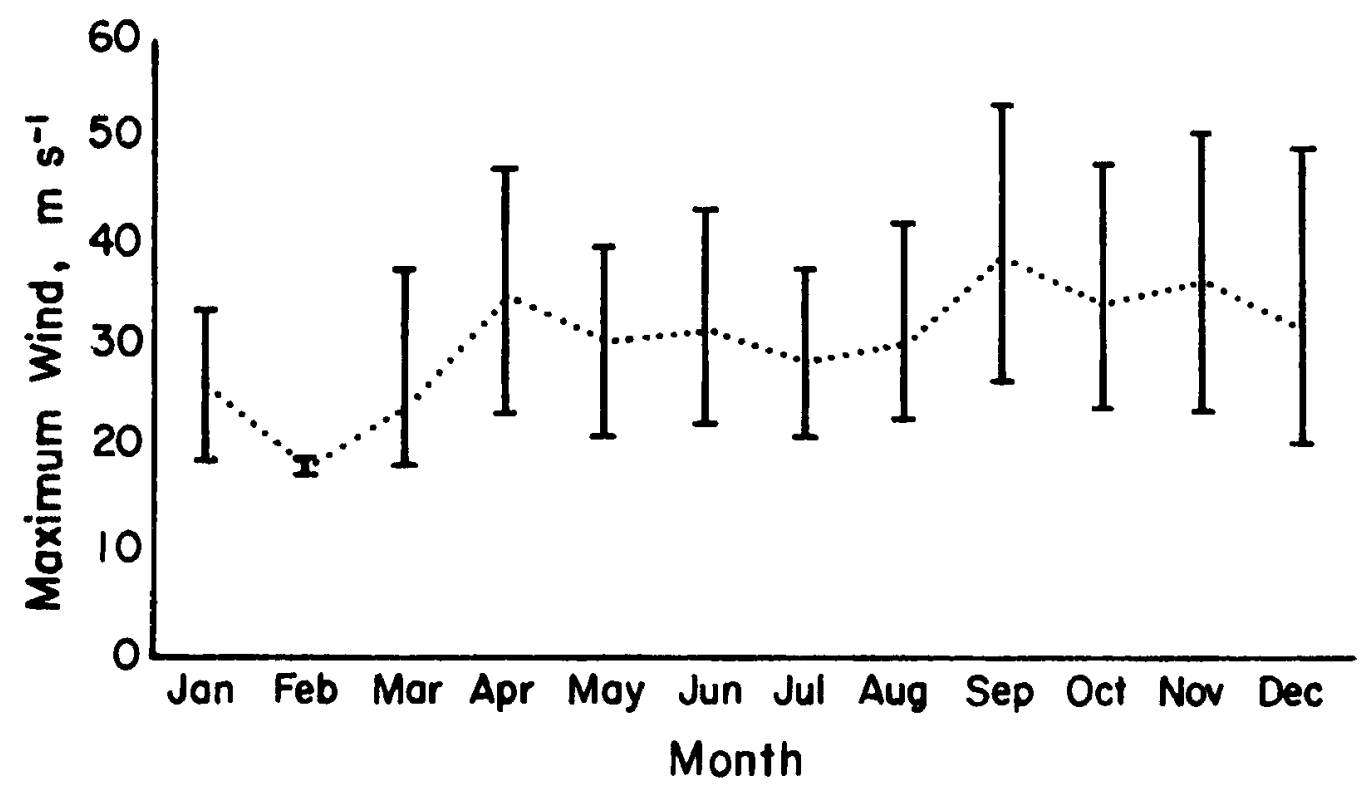

Fig. 11. Same as Fig. 10 but for the Pacific tropical cyclones, 19611969. 
and Reiter, 1980). An examination of the size and track data for both the Atlantic and Pacific reveals similar areas where large or small cyclones typically occur; these areas also change seasonally.

To examine spatial variability, it is necessary to tabulate cyclone occurrences within relatively small regions (5 degree latitude-1ongitude cells are used here). When monthly tabulations are made, not enough cyclone cases are available in some areas to insure a stable mean or median. The alternative approach chosen was to divide the cyclones more coarsely into three categories: 'smal1', 'medium' or 'large', based on size, and subdivide each of these groups by intensity, either 'tropical storm' or 'hurricane/typhoon', for a total of 6 categories in each basin. The criteria for the categories are shown in Table 3 , and the percent frequency of occurrence of each category is given in Table 4.

\section{TABLE 3}

Criteria used in classifying tropical cyclones by size and intensity in the north Atlantic and northwest Pacific.

\section{INTENSITY}

ATLANTIC

Tropica1

Storm

Designated by Nationa1

Hurricape Center, and $17 \mathrm{~m} \mathrm{~s}^{-1}<V_{\max }<33 \mathrm{~m} \mathrm{~s}^{-1}$

$\underset{\text { Typhoon }}{\text { Hurricane/ }} \mathrm{V}_{\max }>33 \mathrm{~m} \mathrm{~s}^{-1}$

$\mathrm{V}_{\max }=$ Maximum Sustained Wind

MSLP = Minimum Sea Love1 Pressure

\section{PACIFIC}

Designated by Joint

Typhoon Warning

Center, and

MSLP > $980 \mathrm{mb}$

Typhoon MSLP $<980 \mathrm{mb}$

\section{SIZE}

Sma11

Medium

Large

ROCI = Radius of Outer Closed Isobar 


\section{TABLE 4}

Frequency of occurrence of size and intensity categories as percentages of basin totals for the north Atlantic and northwest Pacific.

\section{ATLANTIC}

\begin{tabular}{|c|c|c|c|c|}
\hline & Sma 11 & Medium & Large & All Sizes \\
\hline $\begin{array}{l}\text { Trop. Storm } \\
\text { Hurricane }\end{array}$ & $\begin{array}{l}23.2 \\
15.6\end{array}$ & $\begin{array}{l}11.6 \\
18.4\end{array}$ & $\begin{array}{r}9.1 \\
22.1\end{array}$ & $\begin{array}{l}43.9 \\
56.1\end{array}$ \\
\hline A11 Intensities & 38.8 & 30.0 & 31.2 & 100.0 \\
\hline
\end{tabular}

\section{PACIFIC}

\begin{tabular}{|c|c|c|c|c|}
\hline & Sma 11 & Medium & Large & A11 Sizes \\
\hline $\begin{array}{l}\text { Trop. Storm } \\
\text { Typhoon }\end{array}$ & $\begin{array}{l}24.8 \\
10.5\end{array}$ & $\begin{array}{l}19.7 \\
19.6\end{array}$ & $\begin{array}{r}8.4 \\
17.0\end{array}$ & $\begin{array}{l}52.9 \\
47.1\end{array}$ \\
\hline A11 Intensities & 35.3 & 39.3 & 25.4 & 100.0 \\
\hline
\end{tabular}

The intensity criteria were chosen for consistency with the operational designation 'hurricane' in the Atlantic, and the separation for the 'typhoon' rawinsonde composite (Frank, 1977) in the Pacific. In the northwest Pacific, a pressure of $980 \mathrm{mb}$ typically corresponds to a maximum wind of $30-35 \mathrm{~m} \mathrm{~s}^{-1}$ (Atkinson and Holliday, 1977; Drorak, 1975) which is close to the operational separation (based on maximum wind) at $33 \mathrm{~m} \mathrm{~s}^{-1}$. The size criteria were chosen so as to divide the cyclones of each basin into roughly equal thirds.

Monthly percent frequencies of these categories (the percentage of cyclones in a given month which fall into a specific category) display the same seasonal trends as the quantile time series presented in Figs. 8-11. Two examples from the Atlantic basin are shown in Figs. 12 and 13. These figures show the percentage of all tropical cyclones in a given month which are of hurricane intensity (Fig. 12) or are 1arge 


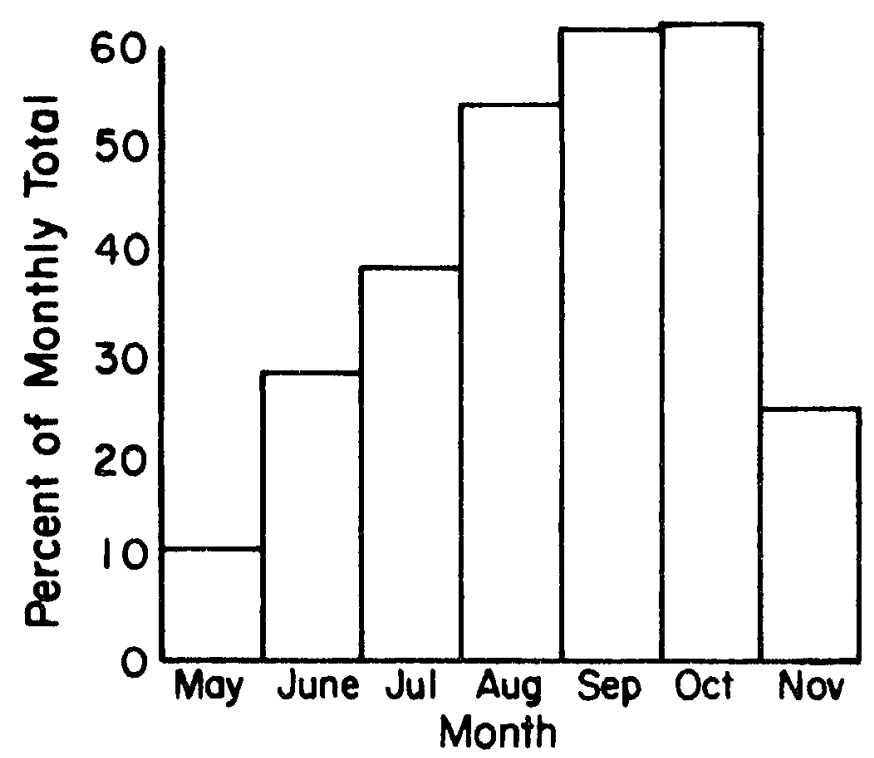

Fig. 12. Monthly percent frequencies of hurricanes for the Atlantic basin, 1957-1977.

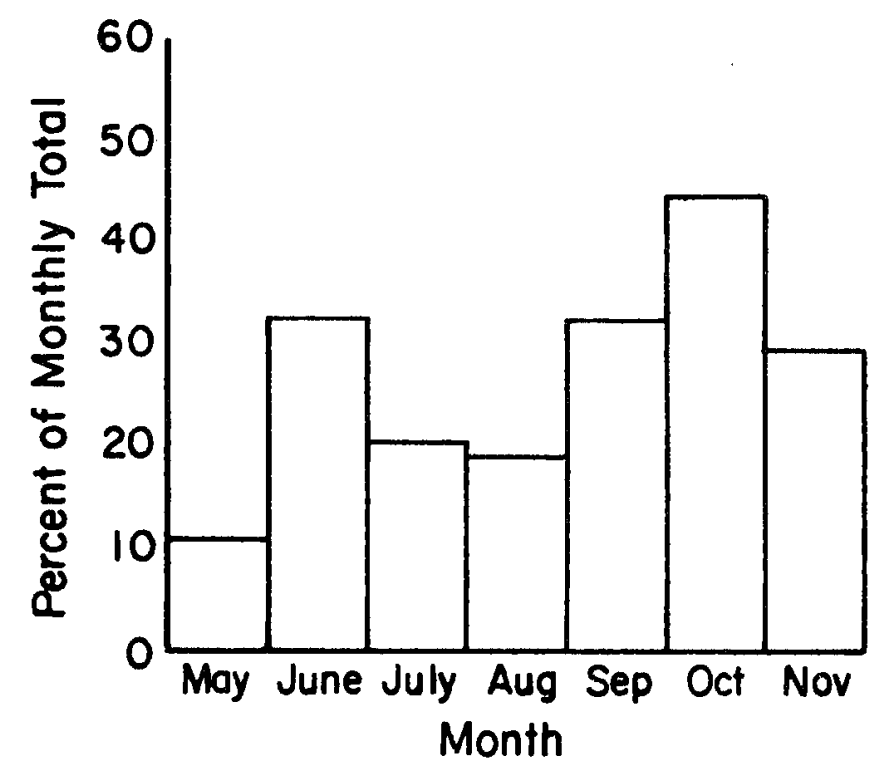

Fig. 13. Monthly percent frequencies of large tropical cyclones (hurricanes and typhoons) for the Atlantic basin, 1957-1977. 
(Fig. 13). What is indicated is the percent frequency of a particular type in a given month. Note that the September-0ctober intensity maximum and October size maximum discussed oarlier are again ovident. Maps of spatial relative frequencies will be used to examine geographical variability of cyclone size.

Figures 14-16 show the percent frequencies of large cyclones for the northwest Pacific for the months of August, September and October. Corresponding maps for the north Atlantic are shown in Figs. 17-19. Percent frequencies are computed for a cell only if the total number of cyclone positions is five or more. The Angust-0ctober period is presented for two reasons: 1) sufficient cases are available over a large area of the basin, and 2) the period covers a transition from a month of sma11 cyclones (August) to a month of large ones (October).

The August-0ctober period in the Pacific will be discussed first since the two existing studies of cyclone size (Arakawa, 1950; Brand, 1972) concern this basin. The August chart in Fig. 14 shows a comparatively broken pattern, but an area of enhanced large cyclone frequency can be seen east of the Philippines, and an area of suppressed large cyclone frequency south and southwest of Japan. Brand (1972) and Arakawa (1950) have both mentioned the latter area as a region where very sma11 typhoons tend to occur, especially in August. The size data for 1961-1969 support this observation; for the region bounded by $25 \mathrm{~N}-$ $35 \mathrm{~N}$ and $125 \mathrm{E}-135 \mathrm{E}, 32$ percent of the 81 cyclone positions were of the 'small typhoon' classification. For the entire basin for the month of Augast, small typhoons comprise 15 percent of the total, and on a yearly basis they account for only 10 percent. Arakawa attributed their formation to the convergence zone which develops when the western end of 


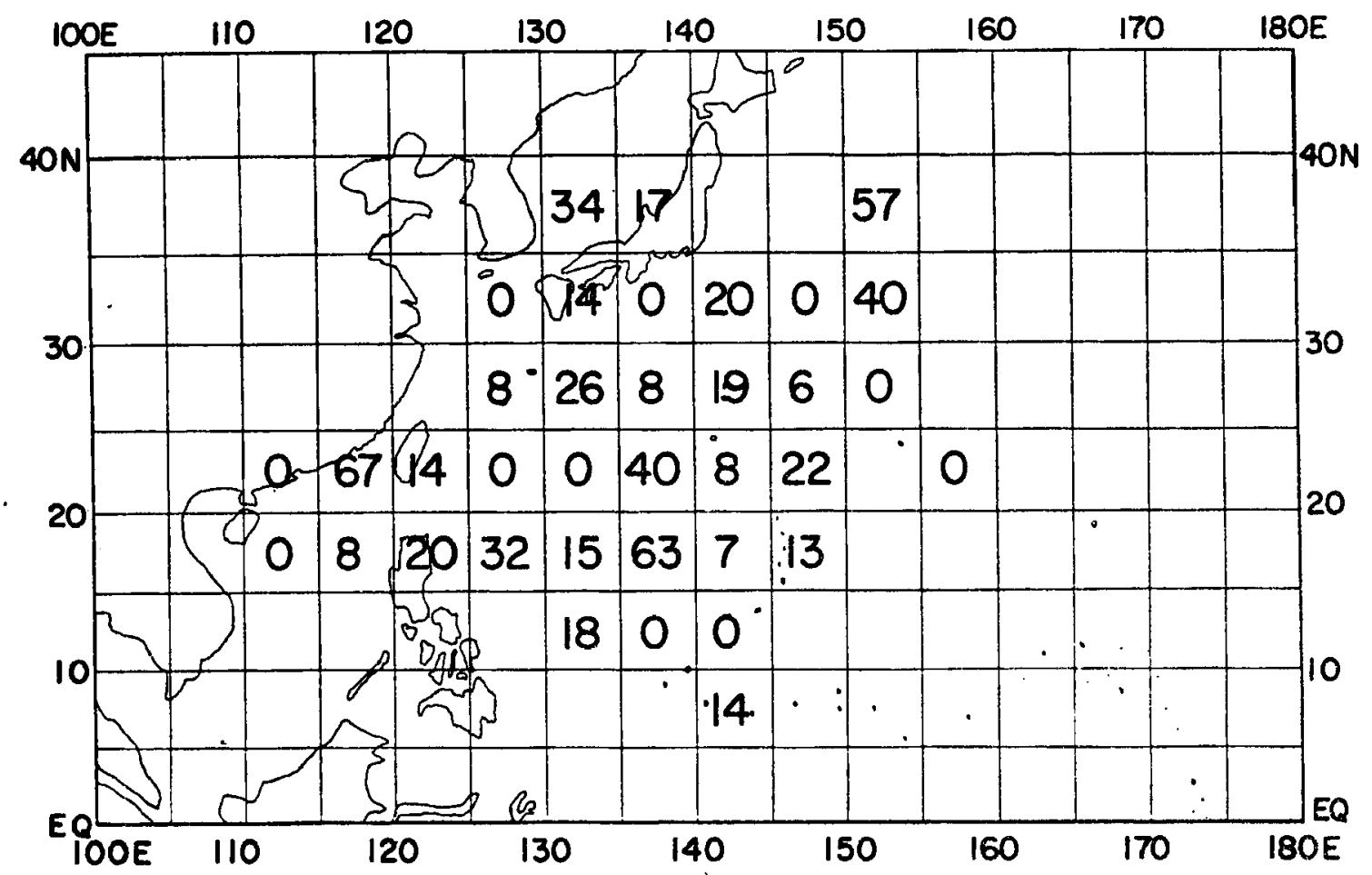

Fig. 14. Percent of cyclones per $5^{\circ}$ latitude-longitude which

fall into the 'large' category. Sample includes all August tropical cyclones, 1961-1969.

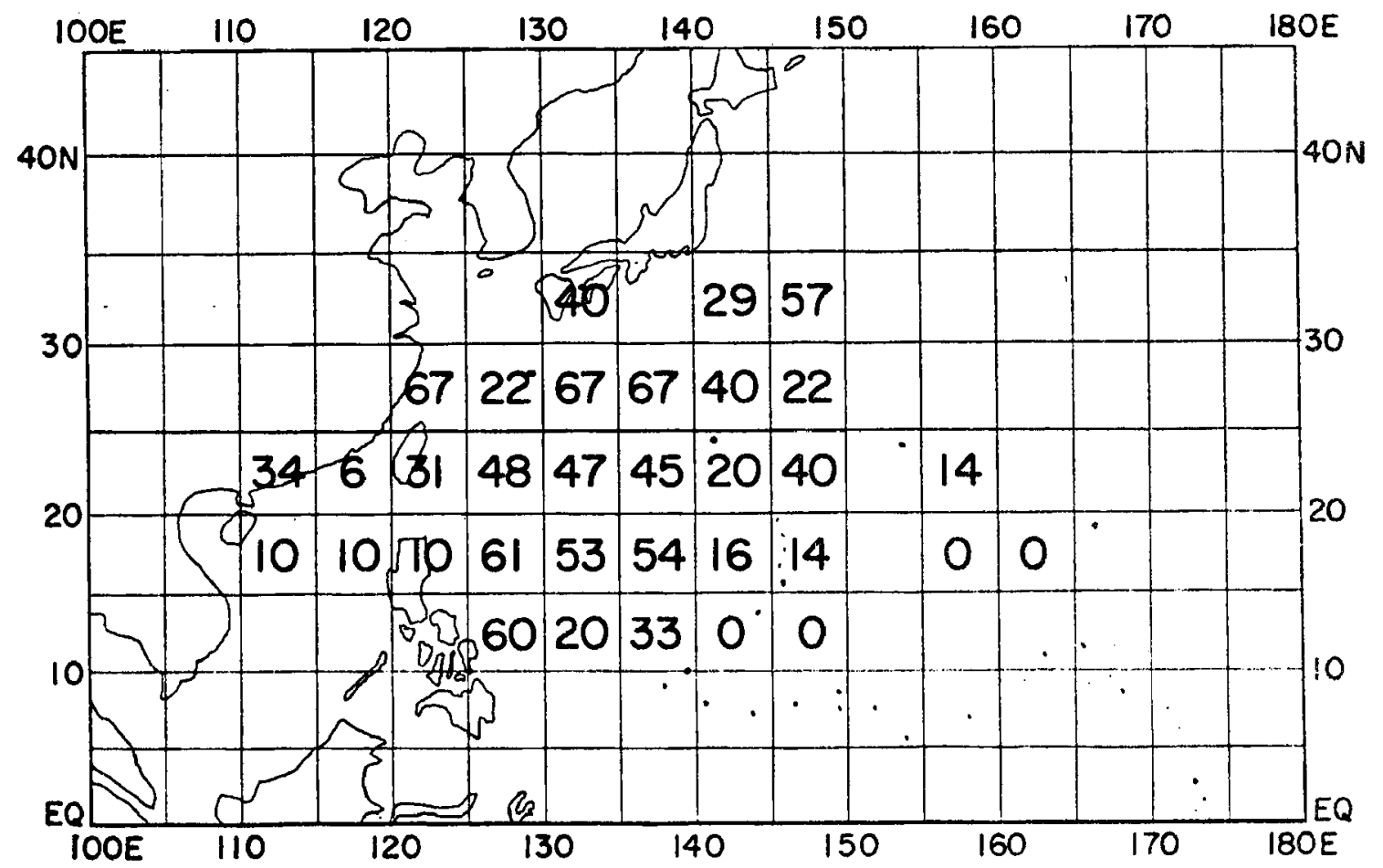

Fig. 15. Same as Fig. 14 except for September tropical cyclones. 


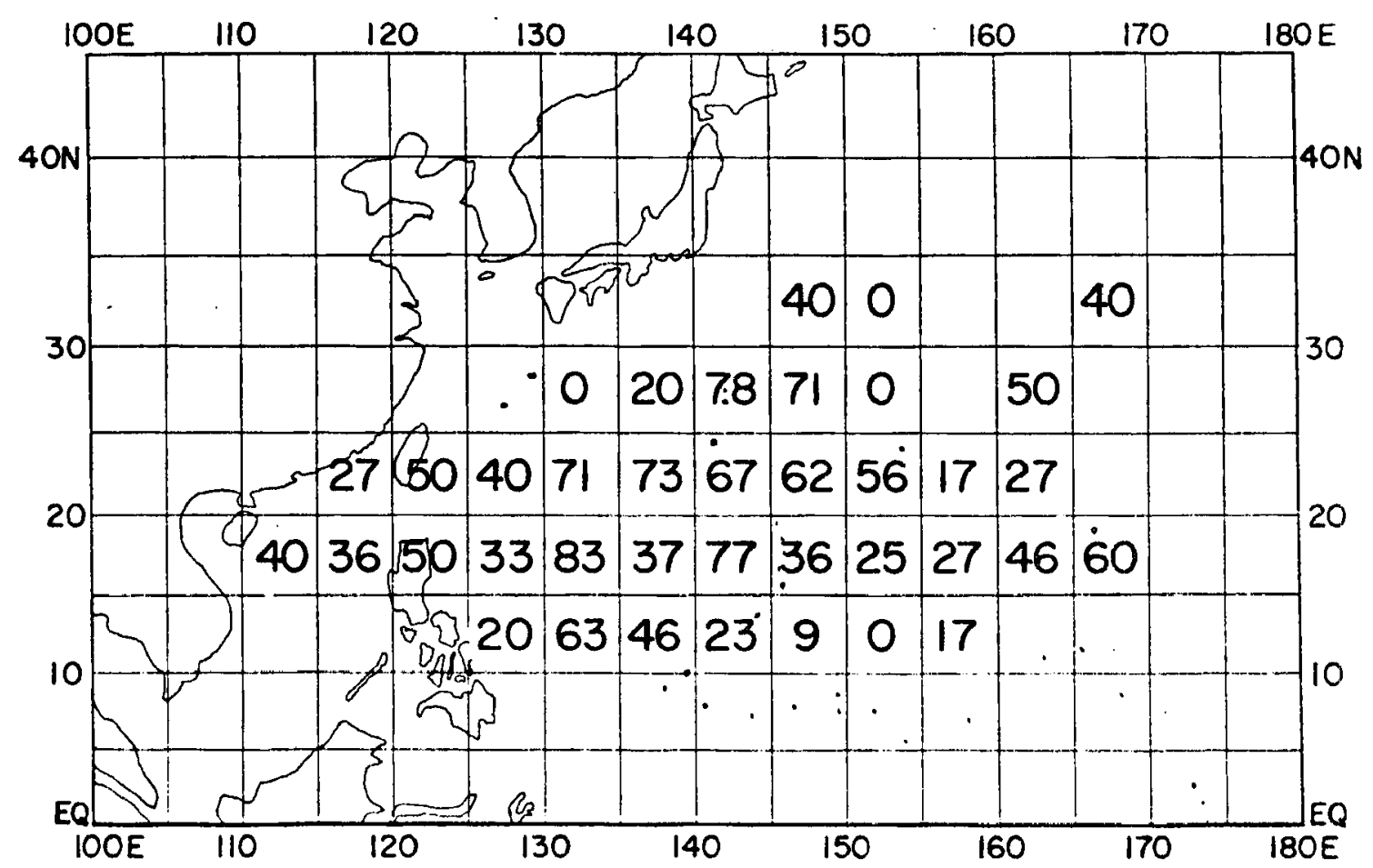

Fig. 16. Same as Fig. 14 except for October tropical cyclonos.

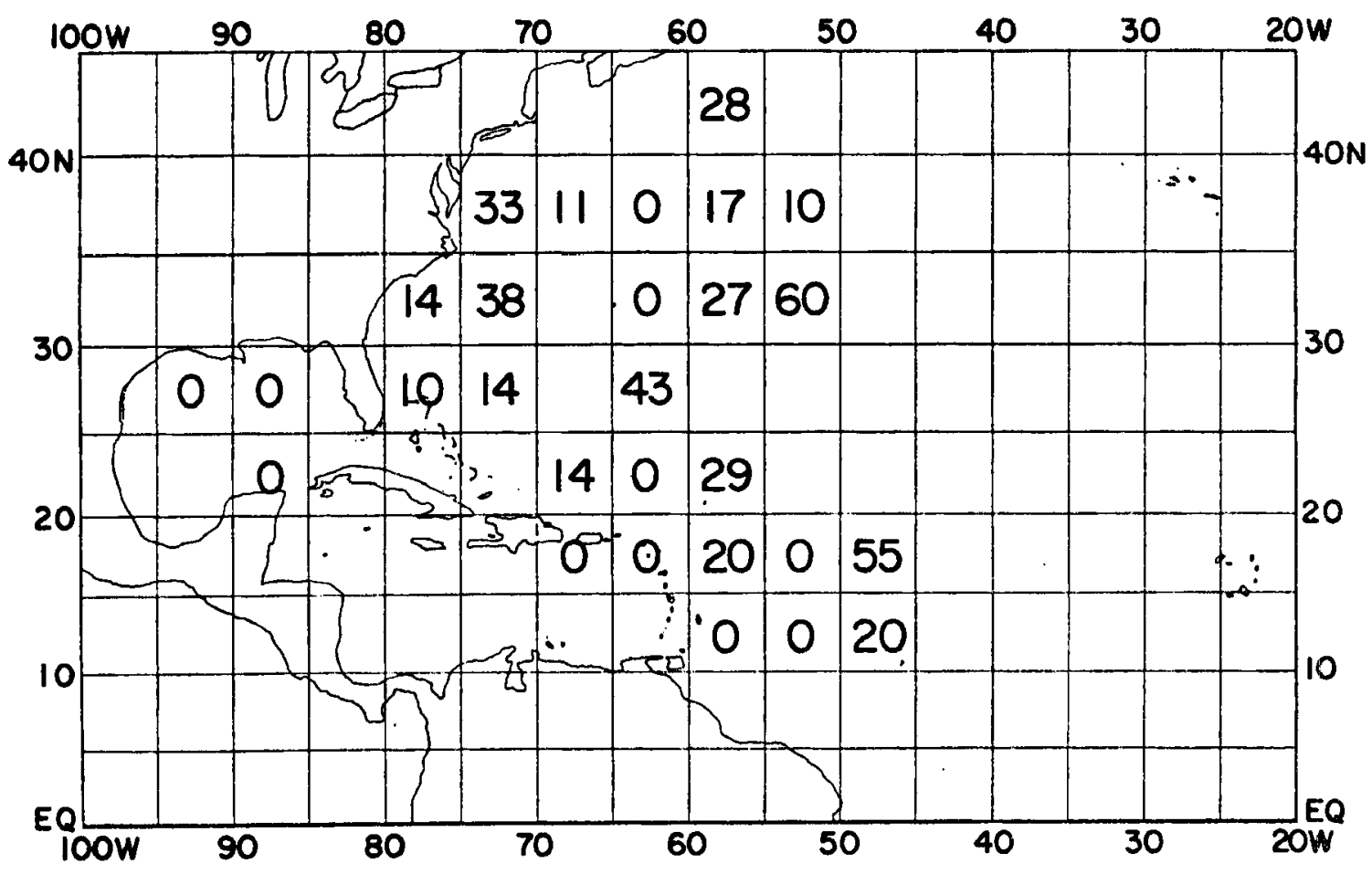

Fig. 17. Same as Fig. 14 except for large August cyclones in the Atlantic basin, 1957-1977. 


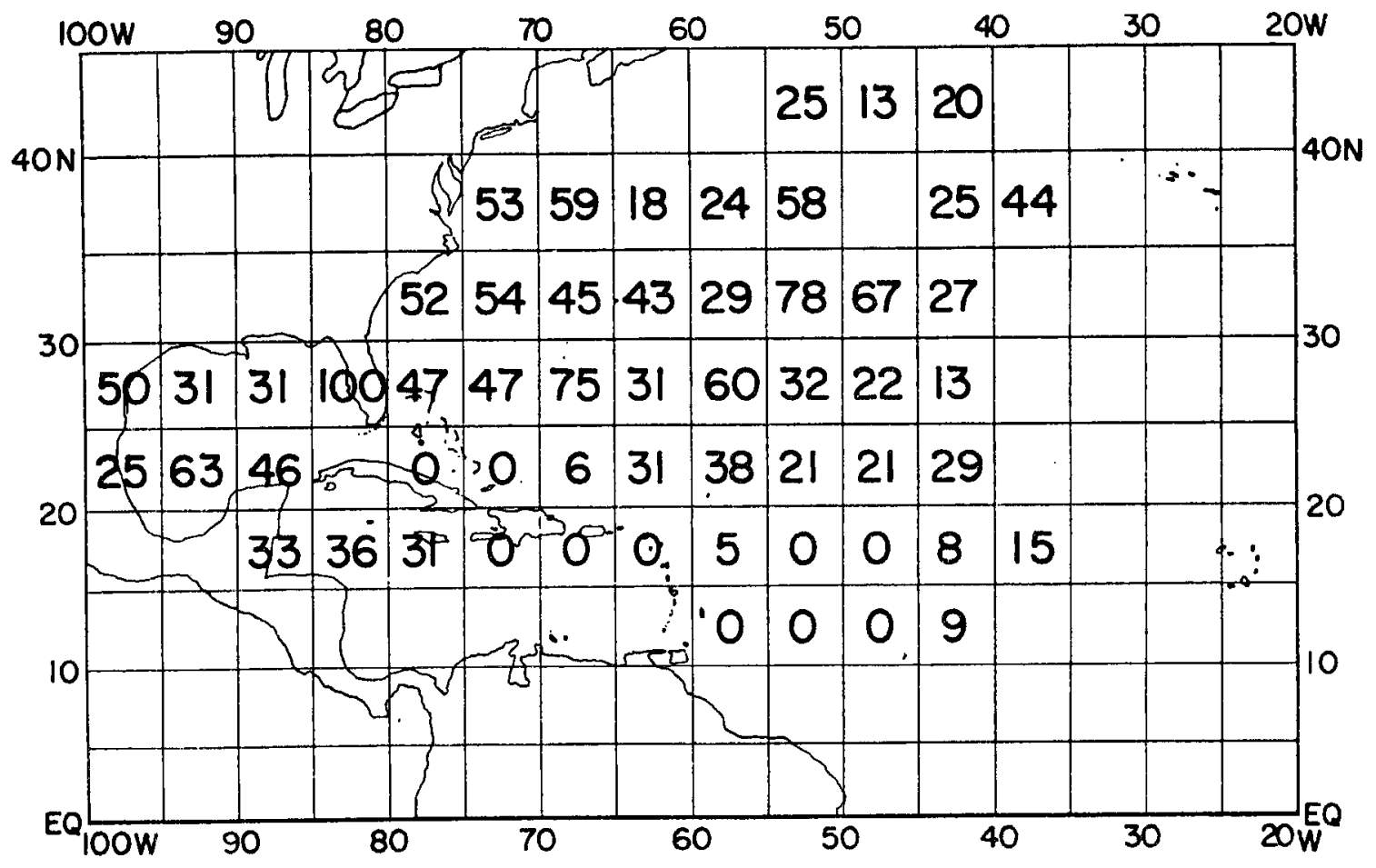

Fig. 18. Same as Fig. 17 except for September tropical cyclones.

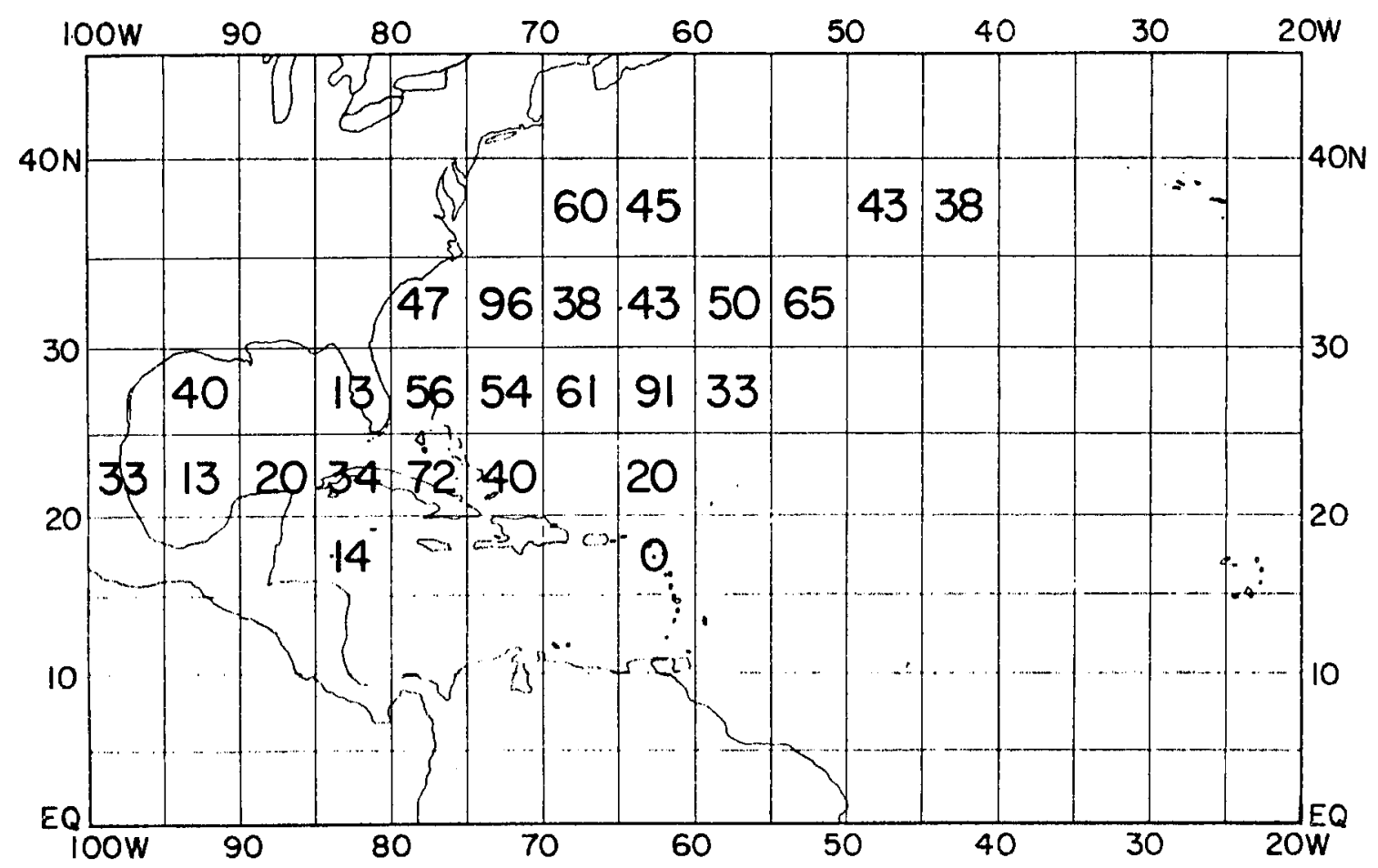

Fig. 19. Same as Fig. 17 except for October tropical cyclones. 
the subtropical ridge fractures and drifts northwestward over the Sea of Japan. The result is a convergent but low-vorticity environment which spawns the sma11 typhoons. The more frequent occurrence of large cyclones east of the Philippines corresponds with the typical location of the monsoon trough, which tends to develop cyclones of relatively large size.

Figure 15 shows frequencies of large cyclones for September. In contrast with August, the area south of Japan is dominated by large cyclones, and the high-frequency region associated with the monsoon trough has become more pronounced. The only areas where large cyclones are relatively rare are the South China Sea and the area south of $20 \mathrm{~N}$ and east of $140 \mathrm{E}$. The change in the frequency of large cyclones in the area south of Japan is thought to be associated with the reversal of the low-level flow which occurs over east Asia beginning in September; the area changes from a region of low vorticity on the margin of a subtropical high to a region of high vorticity between the subtropical ridge and a developing polar high over the continent. By October, 'the month of large cyclones', the region of maximum frequency has shifted southeastward, as the cold surges off of Asia become more frequent and powerfu1 (Fig, 16). Brand (1972) describes an area bounded by $15 \mathrm{~N}-25 \mathrm{~N}$ and $140 \mathrm{E}-145 \mathrm{E}$ as a preferred area for very large typhoons having a ROCI of 10 degrees or more. The frequency of large typhoons (ROCI $\geq 6$ degrees) in this area based on the 1961-1969 sample is 64 percent, as compared with a monthly percentage for the whole basin of 32 percent and a yearly average of 17 percent.

Atlantic. The frequency pattern of large cyclones for the Atlantic basin evolves in a similar fashion, but with one important difference: 
the absence of a monsoon trough means that large cyclones are relatively rare south of $20 \mathrm{~N}$ in all months. In Augnst (Fig. 17), the pattern is somewhat exratic, but large cyclones are most frequent in the subtropical Atlantic from $30 \mathrm{~N}$ to $35 \mathrm{~N}$. Of the 19 cyclone cases in the Gulf of Mexico during August, none were large. This area is similar to the sma11-typhoon region south of Japan, in that the environmental flow is anticyclonic, southerly and relatively weak. In September (Fig. 18) large cyclones become more frequent over the entire basin but particularly in the subtropical Atlantic from $25 \mathrm{~N}-40 \mathrm{~N}$. As in the Pacific, this area begins to be affected by polar outbreaks during September, resulting in a high-vorticity environment. Un1ike the Pacific, the Atlantic has no monsoon trough in Soptember, and the tropics south of $20 \mathrm{~N}$ rarely experience a large cyclone. Note the change of large cyclone frequency from south of $20 \mathrm{~N}$ (rare) to north of $20 \mathrm{~N}$ (frequent). In October (Fig. 19) the preferred genesis area shifts out of the central tropical Atlantic into the western Atlantic and northwest Caribbean where a monsoon trough is occasionally observed, and large cyclone activity reaches a maximum in an area somewhat to the south of the high frequency area of September.

Examination of the maps of frequency of large cyclones has yielded evidence that large and small cyclones are clustered in space as well as time, and that the clustering is consistent in both the Atlantic and Pacific basins. An increase in the frequency of large cyclones is observed in early autumn as the environment over the western oceans changes from anticyclonic to cyclonic with the beginning of cold outbreaks from the continents of Asia and North Amexica. Section 3.5 
will present some typical cyclone 1 ife histories and summarize the climatology of large and small tropical cyclones.

\subsection{The Relationship Between Size and Intensity}

It was mentioned in the introduction and again earlier in this chapter that cyclone size cannot be explained totally in terms of cyclone intensity, although the two are related. This is illustrated in Figs. 20 and 21, which show size as a function of maximum wind for the 21 year Atlantic and 9 year Pacific cyclone samples respectively. Also plotted is the least-squares regression line computed from the size and maximum wind. A linear relationship does exist, but the percentage of variance explained by it (around $10 \%$ in both cases) is small.

In the short term, particularly in the early stages of vortex development, Zipser (1964) noted that sma11 cyclones tended to intensify more rapidly than weaker ones, at least for the 13 cases he examined. The c1imatological data studied here did not reveal a general difference of intensity change between 1 arge and small cyclones.

\subsection{Characteristic Changes in Tropical Cyclone Size and Intensity}

Thus far we have viewed tropical cyclone size as static - in terms of occurrence in given locations and at given times. However, as with al1 cyclone parameters, it changes as the cyclone moves through different environments. Three collections of time series of cyclone size are shown in Figs. 22-24. Figure 22 shows the evolution of size of relatively large Atlantic cyclones with a ROCI of $4^{\circ}$ or more when they first reach hurricane intensity. During the first several days, most of them contract toward the basin mean value of $3^{\circ}$. Figures 23 and 24 both show the changes of size of small hurxicanes which start with a RocI of $2^{\circ}$ or 1ess, with one difference - Fig. 23 is composed only of hurricanes 


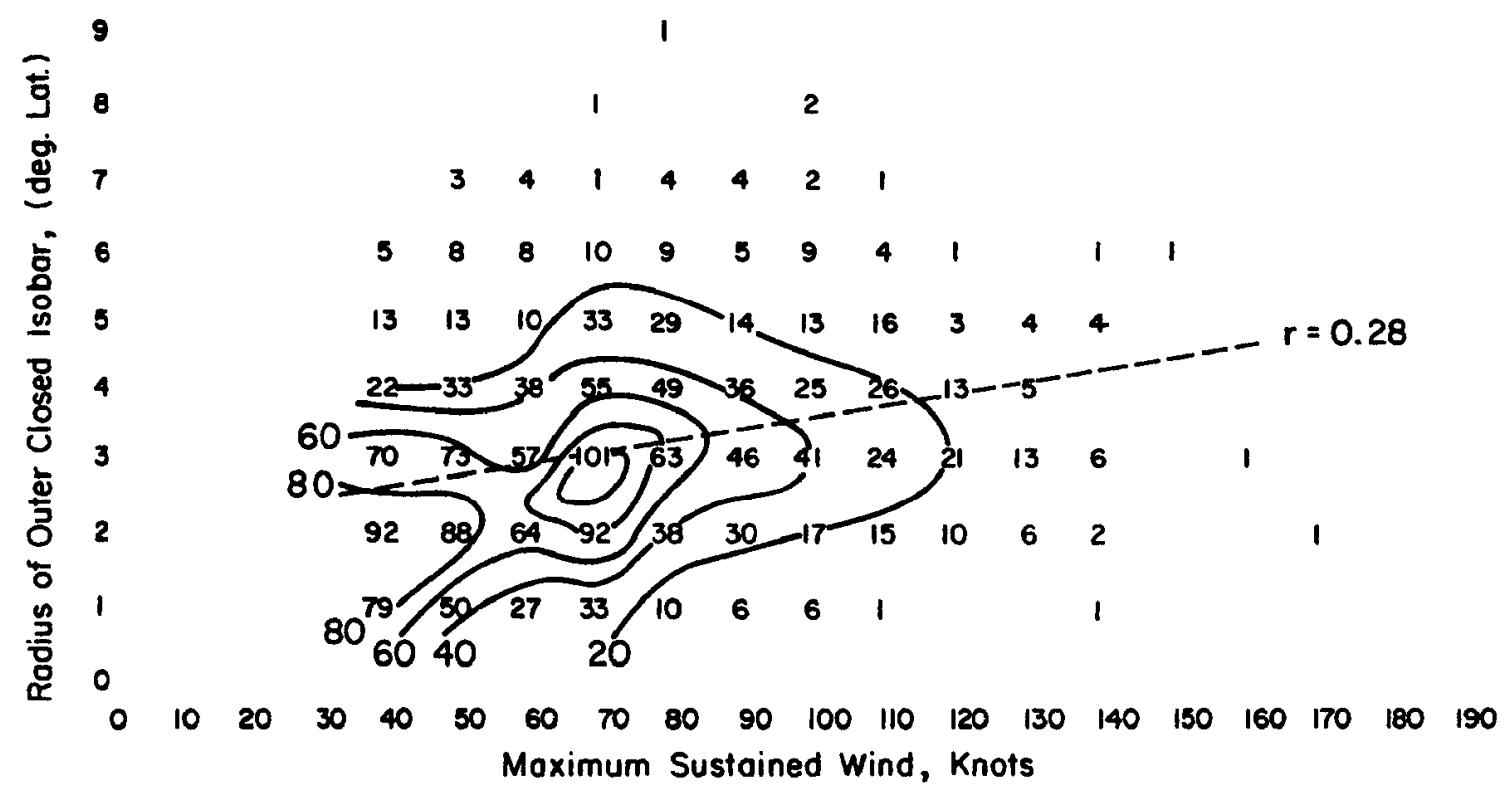

Fig. 20. Tropical cyclone size as a function of maximum sustained wind for Atlantic tropical cyclones, 1957-1977. Observations are tabulated in classes of $1^{\circ}$ latitude and $10 \mathrm{kts}$. The least square 1 ine is fitted to the raw data.

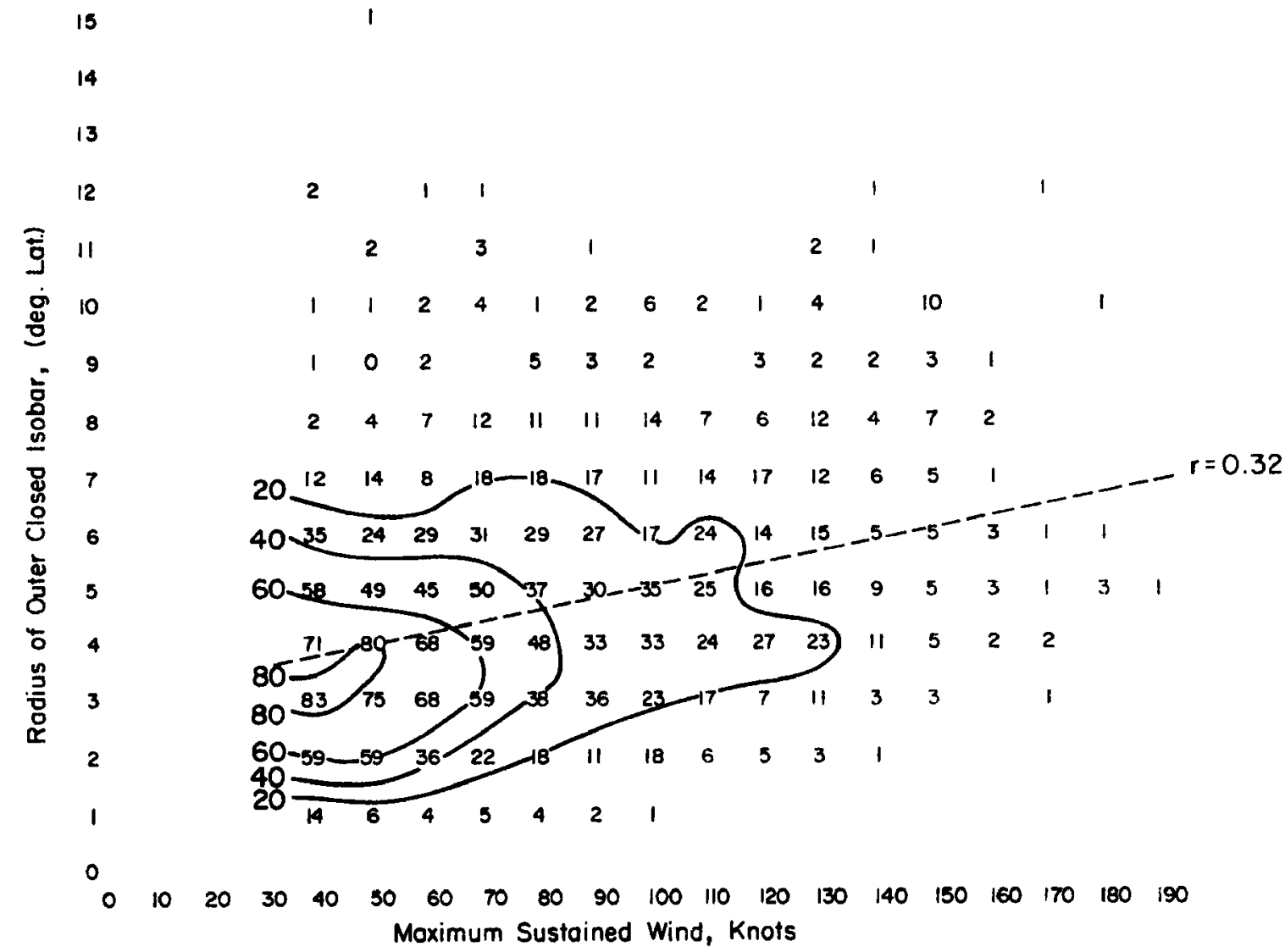

Fig. 21. Same as Fig. 20 except for Pacific tropical cyclones. 


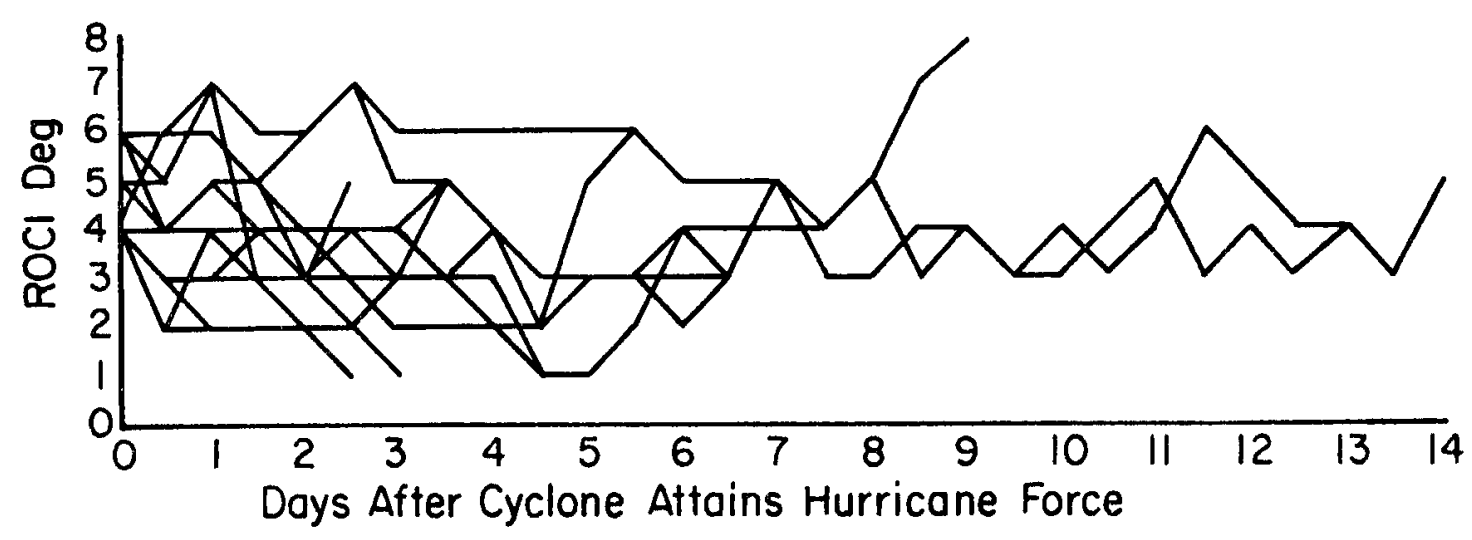

Fig. 22. Changes in size of large hurricanes with an initial RoCI of $4^{\circ}$ 1atitude or more.

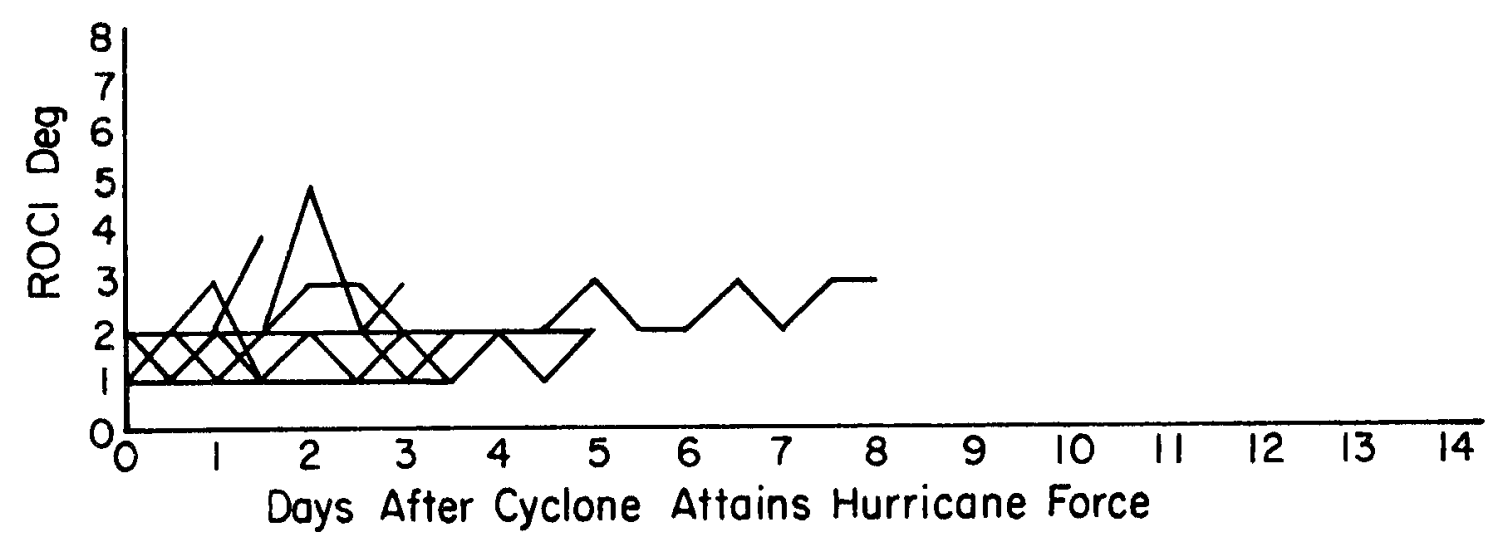

Fig. 23. Changes in size of small westward-moving hurricanes with an initial ROCI of $2^{\circ}$ latitude or less.

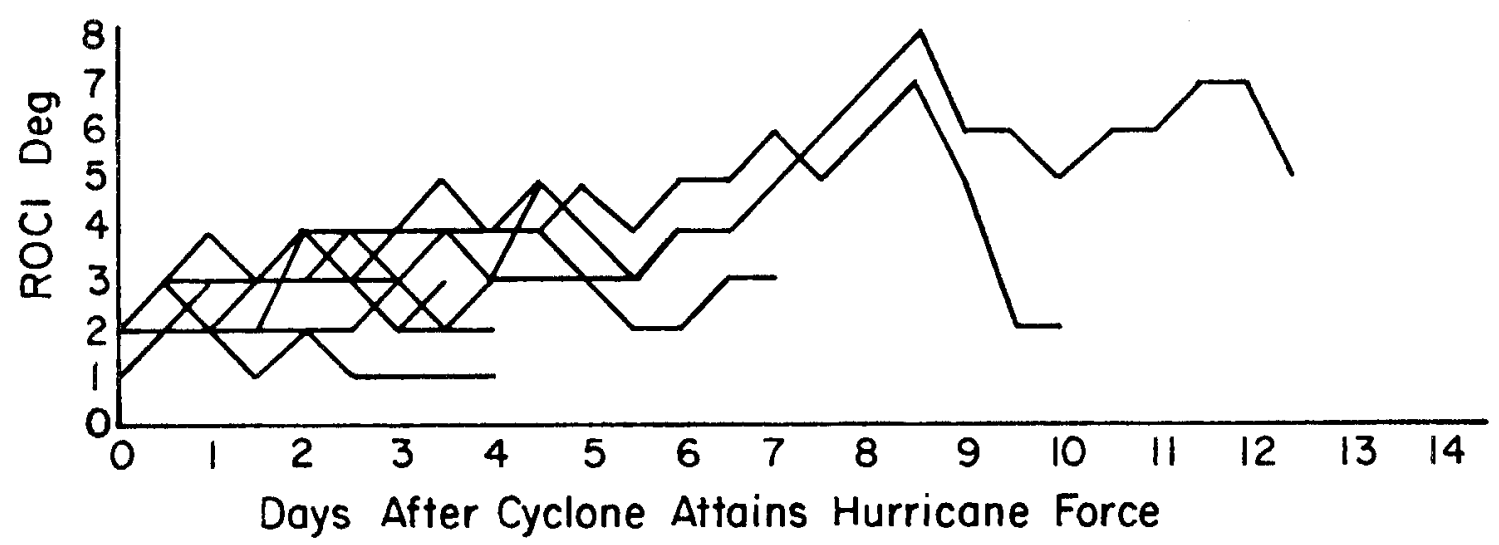

Fig. 24. Changes in size of sma11, recurving or eastward moving hurricanes with an initial ROCI of $2^{\circ}$ latitude or less. 
which moved predominantly westward, while Fig. 24 is of recurving and eastward-moving hurricanes. Westward-moving Atlantic hurricanes (Fig. 23) which begin small tend to remain that way - they never leave the type of environment that spawned them. Recurving systems (Fig. 24) usually grow as they reach the subtropics.

The above implies a characteristic life cycle of hurricanes involving changes in size as well as intensity. Dunn and Miller (1960) and Rieh1 (1979) divided the 1 ife of the Atlantic hurricane into four idealized stages:

1) The formative stage -- a period from the development of the initial vortex to the first occurrence of hurricane force winds. The cyclone typically contracts slightly and develops the characteristic high-energy core during this stage.

2) The immature stage -- a time of rapid intensification with only a very slight change in size. The maximum wind of the cyclone's 1 ife is attained at the end of this stage.

3) The mature stage - the cyclone begins to grow during this stage, but is no longer increasing in intensity. Most of the severe hurricanes affecting the United States are in this stage.

4) The decaying stage -- a period when the cyclone reaches its greatest size and begins to collapse, while the maximum winds diminish.

Such a model of hurricane evolution is useful even though there are many exceptions to it during an actual yclone season. Using the cyclone size and position data, more quantitative versions of this and 
other cyclone life cycles can be prepared by averaging cyclones of a characteristic type. Three are presented here: 1) recurving Atlantic hurricane, 2) October hurricane and 3) October supertyphoon.

The recurving Atlantic hurricane (Fig. 25) follows the 1 ife cycle as described by Rieh1. Twelve hurricanes which formed in the central. Atlantic and attained maximum winds of at least $45 \mathrm{~m} \mathrm{~s}^{-1}$ were averaged together to determine wind and size on successive days after formation. The cyclone forms with a ROCI of 2.5 degrees and remains at about the same size for 3.5 days, and then grows slightly to 4 degrees as it reaches its maximum winds of $60 \mathrm{~m} \mathrm{~s}^{-1}$. It attains a maximum size of 5 degrees one half day after recurvature and 7.5 days after genesis as its winds drop to around $35 \mathrm{~m} \mathrm{~s}^{-1}$. Note that the increase in size commences as the cyclone turns northward, typically into a slowly-moving trough in the westerlies associated with a polar high over the eastern United States.

Figure 26 is a composite of six October hurricanes. Occasionally in June or October the intertropical convergence zone shifts far enough to the north in the western Caribbean Sea to acquire the cyclonic lower tropospheric flow characteristic of a monsoon trough, and a cyclone of this type may develop. They may on occasion become quite severe, but most typically reach maximum winds of $45 \mathrm{~m} / \mathrm{s}$ or $1 \mathrm{ess}$, and remain at nearly the same size or even contract slightly as they move into the Atlantic. Their usual genesis size of 4 degrees is large for the At1antic.

Figure 27 shows the October supertyphoon, characteristically the largest and most powerful tropical cyclone on earth. This cyclone is based on an average of 6 typhoons with central pressures below $930 \mathrm{mb}$. 


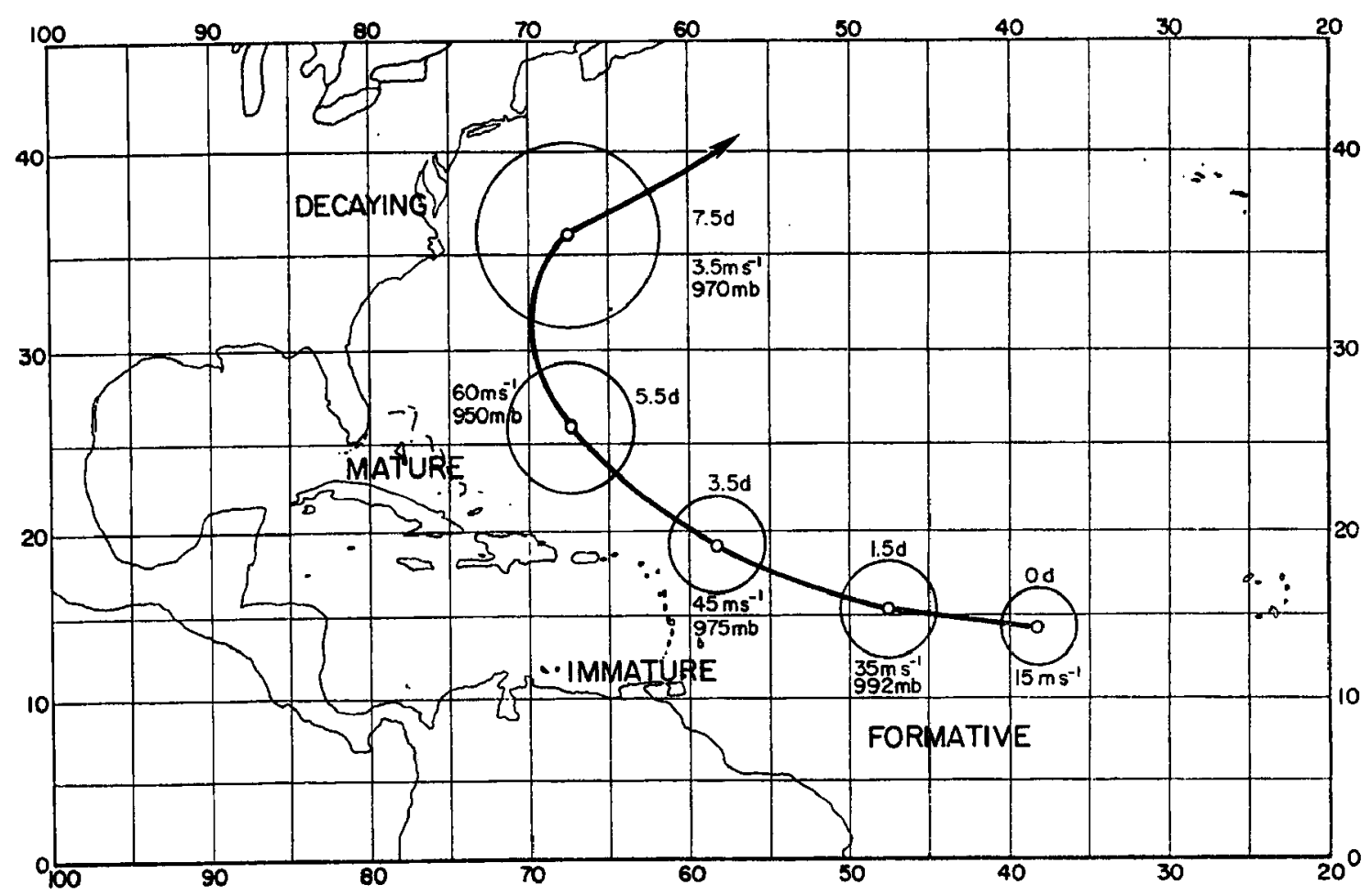

Fig. 25. Average changes of size and intensity of 12 recurving At1antic hurricanes. The circle has the same radius as the average outer closed isobar.

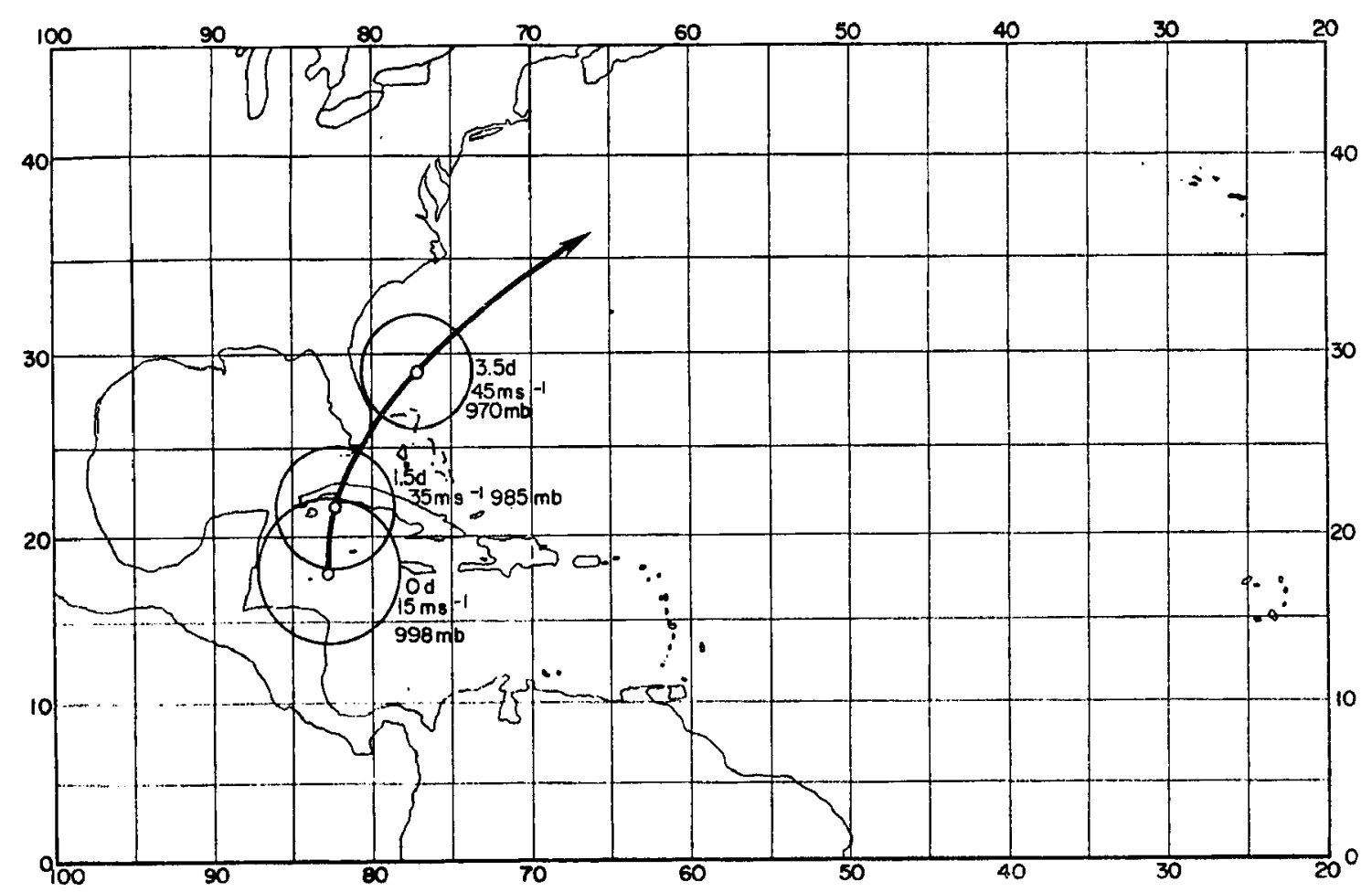

Fig. 26. Same as Fig. 25 except for the six October hurricanes. 


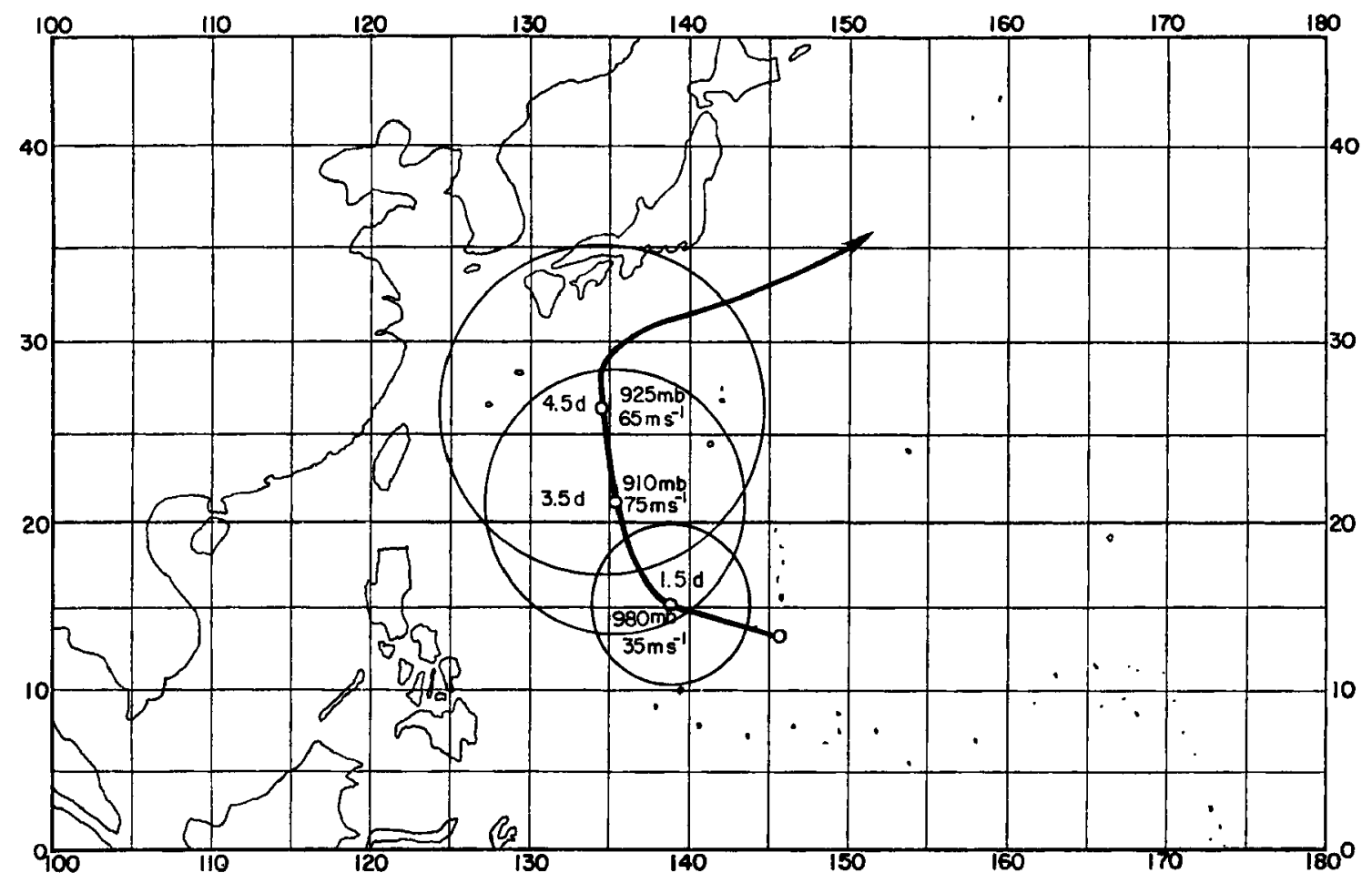

Fig. 27. Same as Fig. 25 except for six October supertyphoons.

It forms at a size of 5 degrees in the monsoon trough, and simultaneously deepens rapidly to $910 \mathrm{mb}$ and grows to 8 degrees. Peak winds reach about $75 \mathrm{~m} \mathrm{~s}^{-1}$. At recurvature it has a ROCI of 10 degrees and winds of about $65 \mathrm{~m} \mathrm{~s}^{-1}$.

These three 1 ife cycles are a very 1 imited representation of the patterns that can evolve. Some of the general conclusions resulting from the c1imatology of large and sma11 tropical cyclones are listed below.

1) Typhoons and Pacific tropical storms are over twice as large as their Atlantic counterparts when the area enclosed by the outer closed isobar is considered. 
2) A consistent, seasonal progression in tropical cyclone size, with a midsummer minimum and fall maximum, is present in both ocean basins.

3) Large cyclones are more frequent in particular regions within the basin where the lower-tropospheric vorticity of the environment is relatively large -- in the vicinity of the monsoon trough or between the subtropical ridge and the polar anticyclones which begin to develop over the eastern United States and China during early antumn.

4) Converse1y, sma11 cyclones are more frequent in areas of minimum environmental vorticity - the trade easterlies of the tropical Atlantic and Pacific, and the subtropical ridge margins of the midsummer Gu1f of Mexico and extreme western Pacific 0cean. 
4. STRUCTURE OF LARGE AND SMALL TROPICAL CYCLONES

\subsection{Introduction}

The previous chapter addressed the first task of this paper by presenting a climatology describing the variability of tropical cyclone size both temporally and spatially. This chapter discusses the structural aspects of cyclone size, including mean wind fields based on rawinsonde compositing methods, differences between mean cyclones of different sizes, basins, and intensities, statistical support for these differences and some approximate spatial distributions of $15 \mathrm{~m} \mathrm{~s}^{-1}$ surface winds.

The rawinsonde compositing method used in this work has been in use on Professor Gray's tropical cyclone research project for several years. For each cyclone position, all available rawinsonde soundings within $15^{\circ}$ latitude are navigated onto a polar-coordinate grid centered on the cyclone. The basic grid (Fig. 28) consists of 8 octants subdivided into 8 belts. For wind compositing, the grid may be positioned in two ways geographically, with octant 1 always to the north, or relative to the cyclone, with octant 1 centered on the cyclone motion vector. The winds themselves can also be modified by subtracting out the cyclone motion vector. Four wind composites result: 1) Natura1, or NAT - geographic grid and total winds, 2) Motion, or MOT - geographic grid and relative winds (cyclone motion removed), 3) Rotated or ROT - rotated grid and natura1 winds, and 4) MOTROT - rotated grid and relative winds. This 


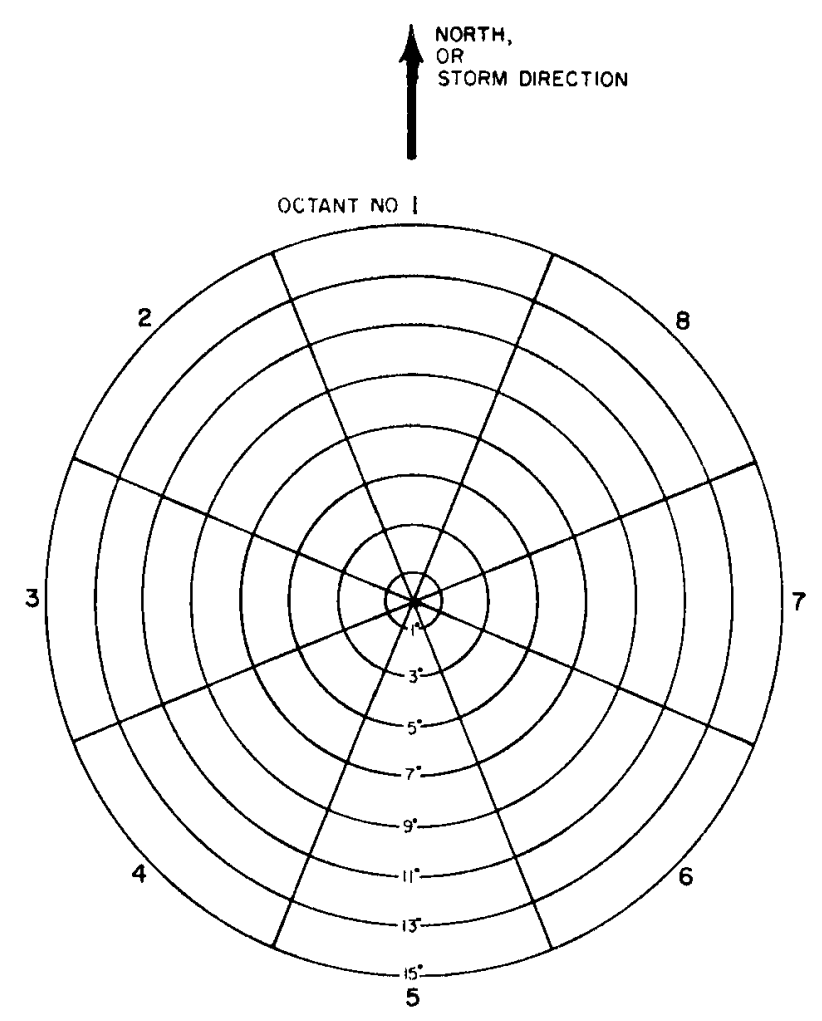

Fig. 28. Grid used in compositing rawinsonde observations around tropical cyclones.

rawinsonde compositing method has been extensively documented by Gray et a1. (1982). The grid is repositioned for each cyclone central position, and an average is computed for each sounding parameter at each of 21 pressure levels from the surface to $50 \mathrm{mb}$ using all soundings in each grid region.

Rawinsonde composites were made for 11 mutually exclusive cyclone position sets from Atlantic and Pacific in order to detect systematic differences between large and small tropical cyclones. The six stratifications for the Pacific are identical to those used to study the c1imatology. The Atlantic composite stratifications have been modified somewhat. Stratifications for both basins are 1isted in Table 5. The changes in the Atlantic stratifications from those used in the 


\section{TABLE 5}

Stratification criteria for large and small tropical cyclones. ATLANTIC

\begin{tabular}{|c|c|c|}
\hline $\begin{array}{l}\text { Sma11/Medium Tropical Storm } \\
\text { Large Tropical Storm } \\
\text { Sma11/Medium Hurricane } \\
\text { Large Hurricane North } \\
\text { Large Hurricane South } \\
\text { PACIFIC } \\
\text { Sma11 Tropical Storm } \\
\text { Medium Tropical Storm } \\
\text { Largo Tropical Storm } \\
\text { Sma11 Typhoon } \\
\text { Medium Typhoon } \\
\text { Large Typhoon }\end{array}$ & $\begin{aligned} & R<3^{\circ} \\
& 4^{\circ} \leq R \leq 5^{\circ} \\
& R \geq 6^{\circ} \\
& R \leq 3^{\circ} \\
& 4^{\circ} \leq R \leq 5^{\circ} \\
& R \leq 6^{\circ}\end{aligned}$ & $\begin{array}{l}17 \mathrm{~ms}^{-1} \leq \mathrm{v}_{\max }<33 \mathrm{~ms}^{-1} \\
17 \mathrm{~m} \mathrm{~s} \leq \mathrm{v}_{\max }<33 \mathrm{~ms}^{-1} \\
\mathrm{v}_{\max } \geq 33 \mathrm{~ms}^{-1} \\
\mathrm{v}_{\max } \geq 33 \mathrm{~ms}^{-1} \text { LAT }>25 \mathrm{~N} \\
\mathrm{v}_{\max } \geq 33 \mathrm{~m} \mathrm{~s}^{-1} \text { LAT } \leq 25 \mathrm{~N}\end{array}$ \\
\hline
\end{tabular}

climatological study were to allow comparisons between Atlantic and Pacific cyclones after the influence of size differences have been removed.

4.2 Mean Structura1 Differences Between Large and Sma11 Tropical Cyclones

The azimuthally averaged tangential winds in NAT coordinates for four Atlantic and four Pacific composites are shown in the Appendix. All are qualitatively similar - a maximum in tangential winds at small radii and in the low to middle troposphere, decreasing upward and becoming negative in the upper troposphere. The intercomparisons which can be made are quite numerous, but w111 here be 1 imited to three types: 1) comparisons of cyclones of equivalent intensity and differing size, 2) comparisons of cyclones of equivalent size and differing intensity, and 3) comparisons of cyclones of equivalent size and intensity from 
different basins. Differences in strength are not treated explicitly but are apparent in many of the comparisons.

Figures 29-32 show difference fields between cyclones of different size but equivalent intensity. With the exception of the Atlantic large hurricane minus the sma11/medium hurricane, the maximum tangential wind differences 1 ie in the low and middle troposphere at radii of $4^{\circ}$ to $6^{\circ}$ 1atitude. In al1 cases the wind differences in this region exceed $5 \mathrm{~m}$ $s^{-1}$ - roughly equivalent to a doubling of wind speeds in this area. Tangential wind difference fields for cyclones of different intensities (Figs, 33-36) reveal the structural imp1ications of size differences and intensity differences. Differences in tangential wind in excess of $5 \mathrm{~m} \mathrm{~s}^{-1}$ are here confined to the region inside $3^{\circ}\left(4^{\circ}\right.$ for large typhoon minus sma11 typhoon), and changes from $4-6^{\circ}$ are on the order of only $2.5 \mathrm{~m} \mathrm{~s}^{-1}$. Even at $2^{0}$ radius, where the typical difference is about $7.5 \mathrm{~m} \mathrm{~s}^{-1}$ a change of only $20 \%-40 \%$ is observed. When it is then considered that the area from $4-6^{\circ}$ radius is over twice as 1 arge as the area from $0-3^{\circ}$ radius, the importance of the wind differences for cyclones of different sizes becomes apparent. Some of the implications of cyclone size on cyclone dynamics will be addressed in Chapter 5 .

A by-product of these size-based composites is the first opportunity to compare hurricanes and typhoons after the size difference has been eliminated. Figure 37 shows the difference between the sma11/medium hurricane and small typhoon - both have a ROCI of $1-3^{\circ}$. Most of the difference indicates that the smal1/medium hurricane is of 


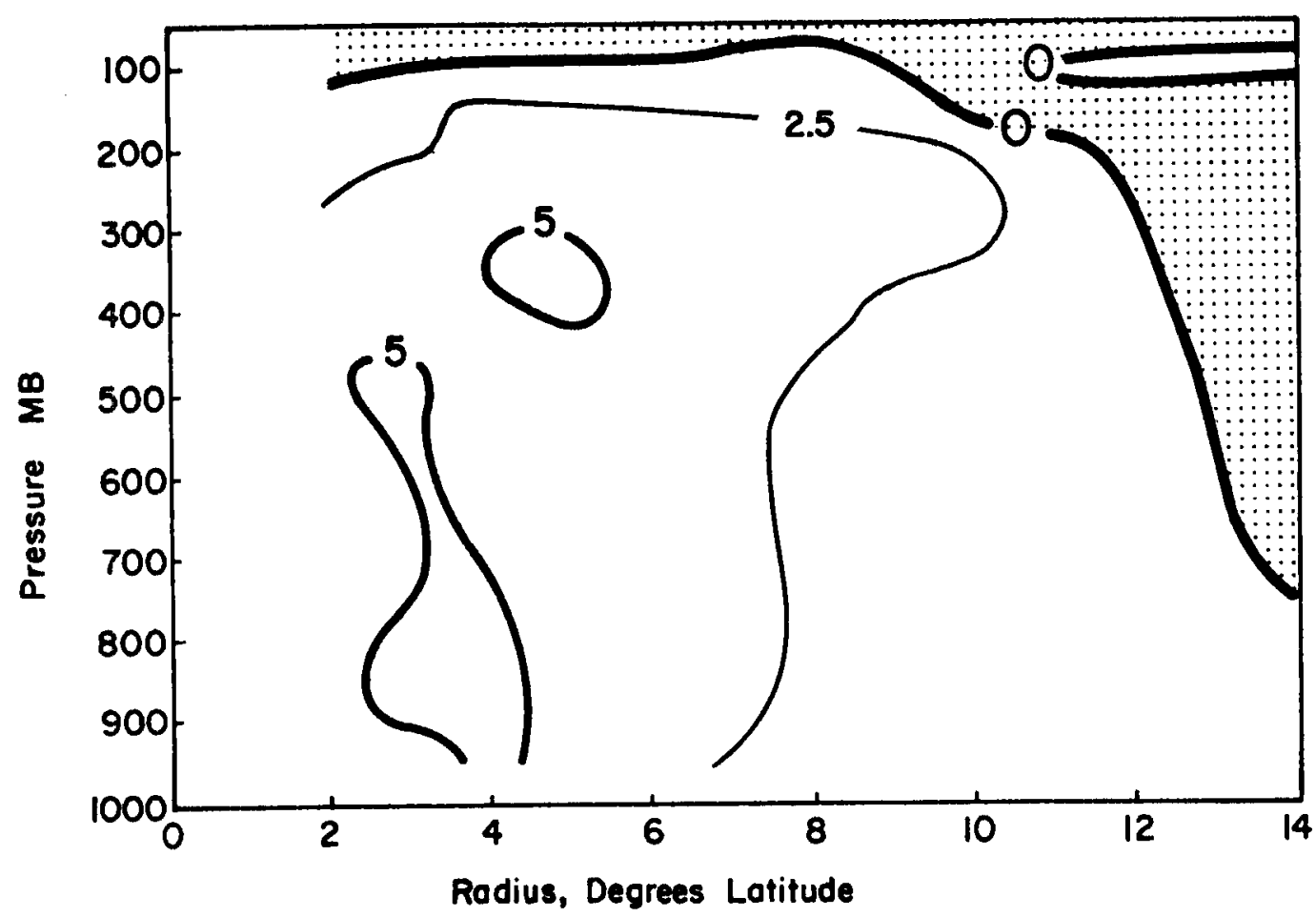

Fig. 29. Difference of azimuthal mean tangential wind, Atlantic large tropical storm minus Atlantic small tropical storm.

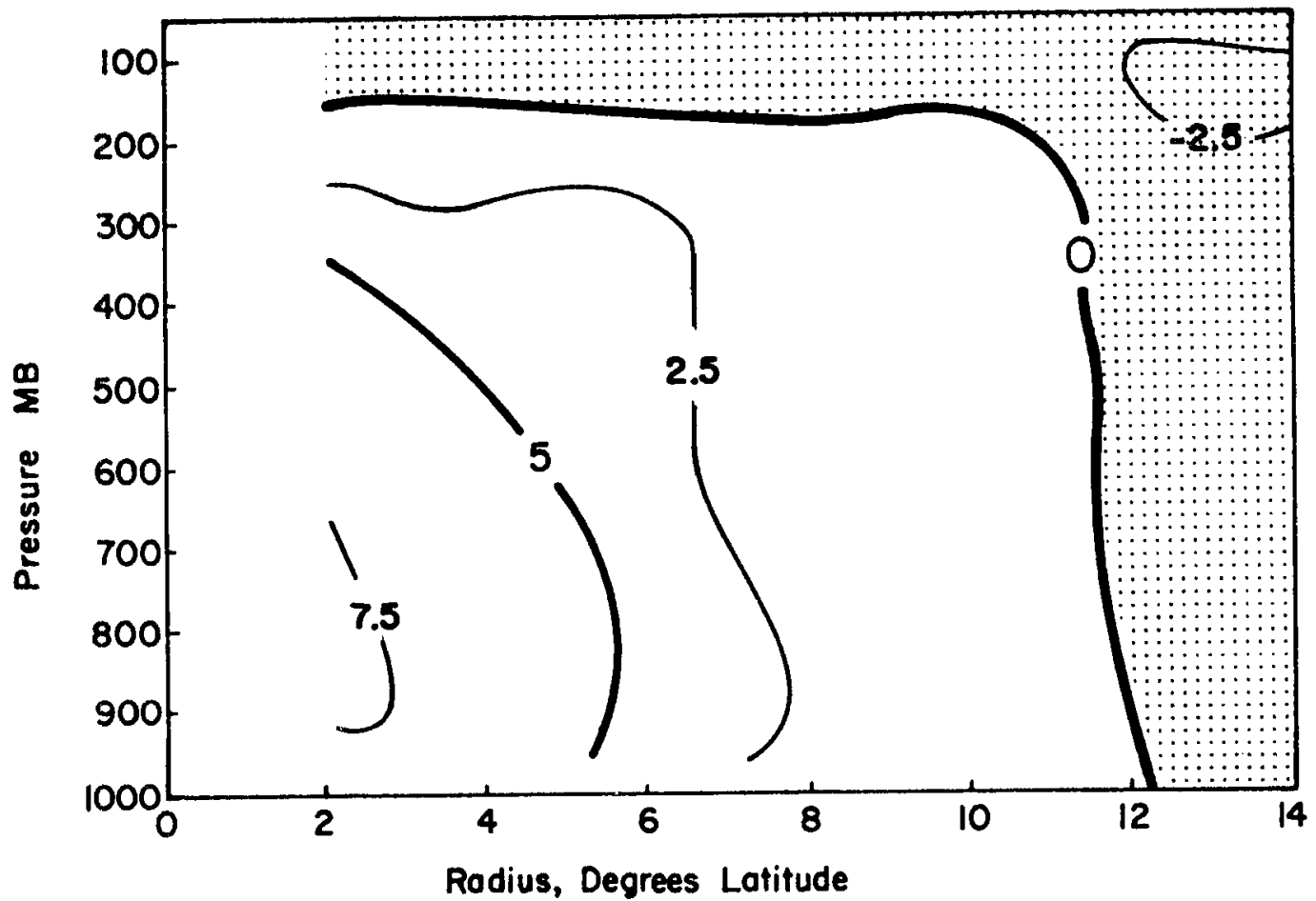

Fig. 30. Same as Fig. 29 except for Atlantic large hurricane north minus sma11/medium hurricane. 


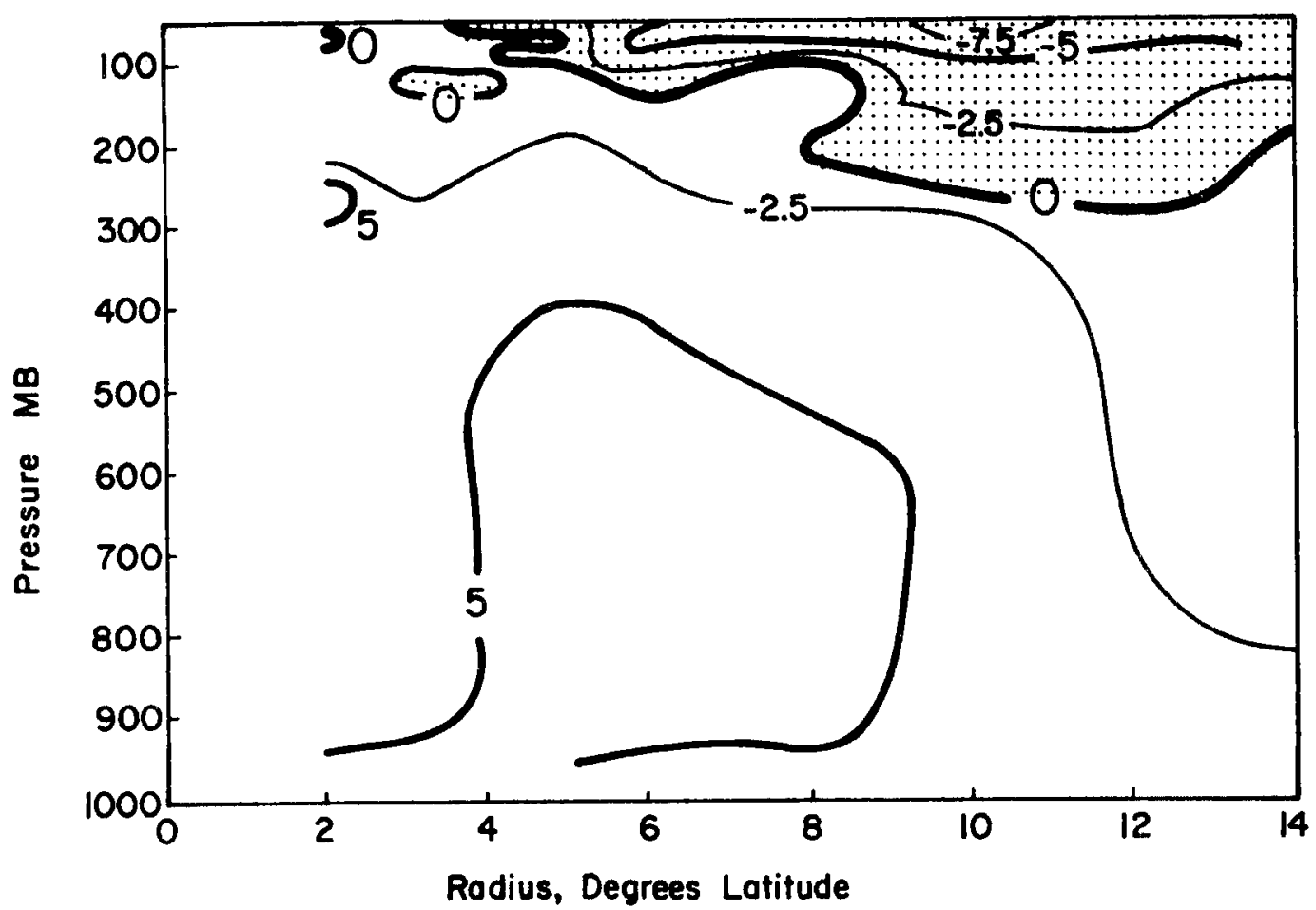

Fig. 31. Same as Fig. 29 except for Pacific large tropical storm minus small tropical storm.

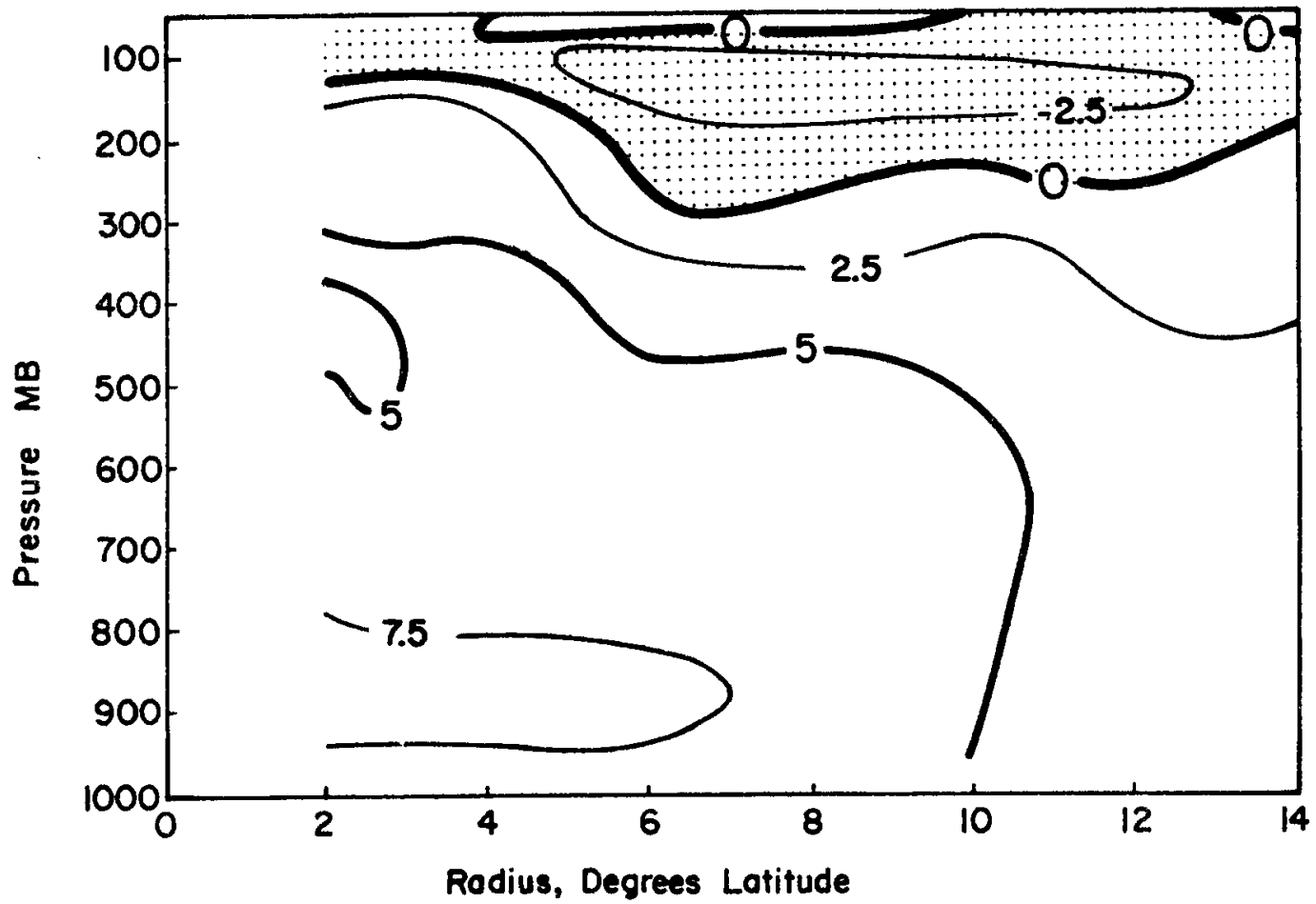

Fig. 32. Same as Fig. 29 except for Pacific large typhoon minus small typhoon. 


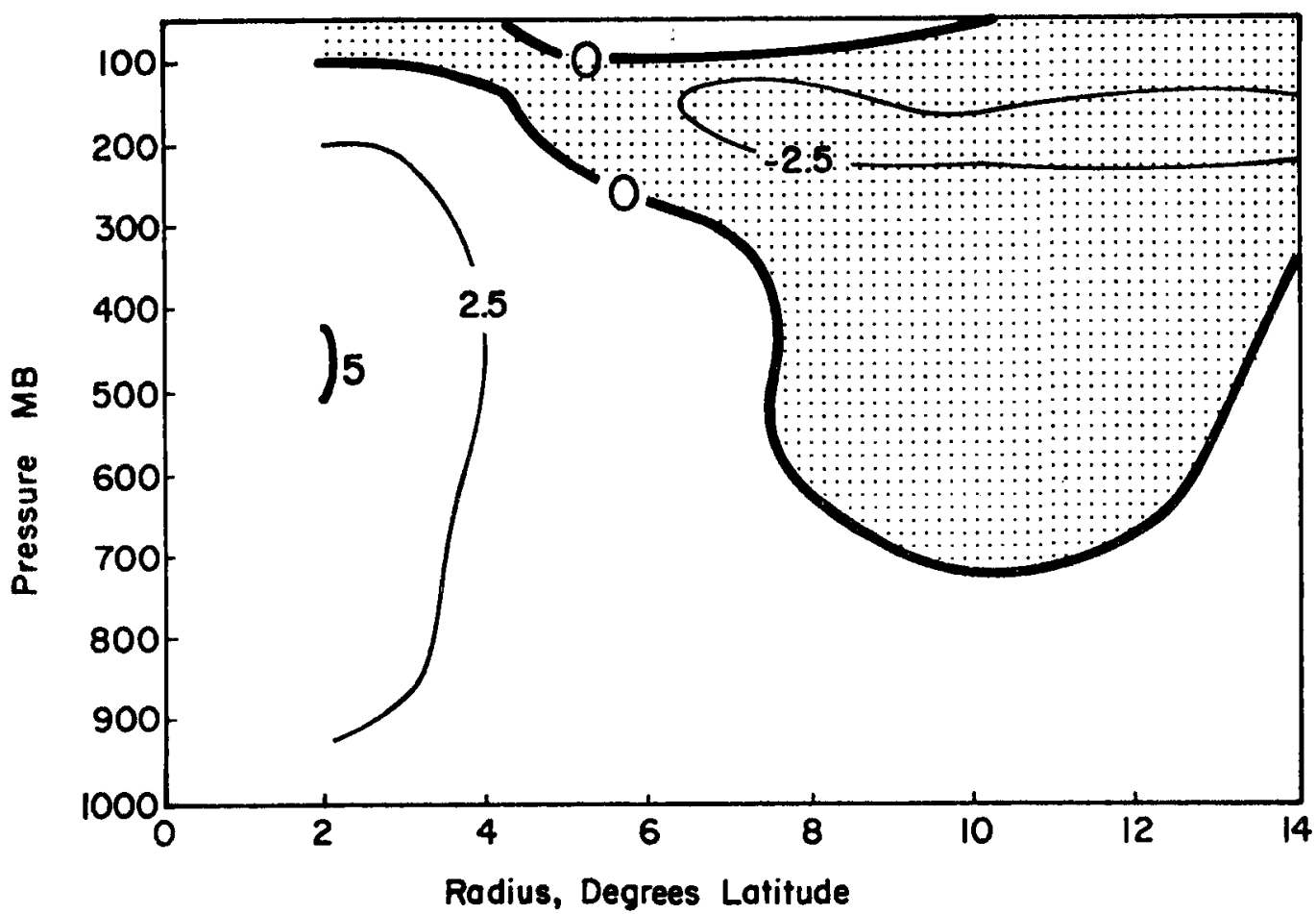

Fig. 33. Same as Fig. 29 except for Atlantic sma11/medium hurricane minus small tropical storm.

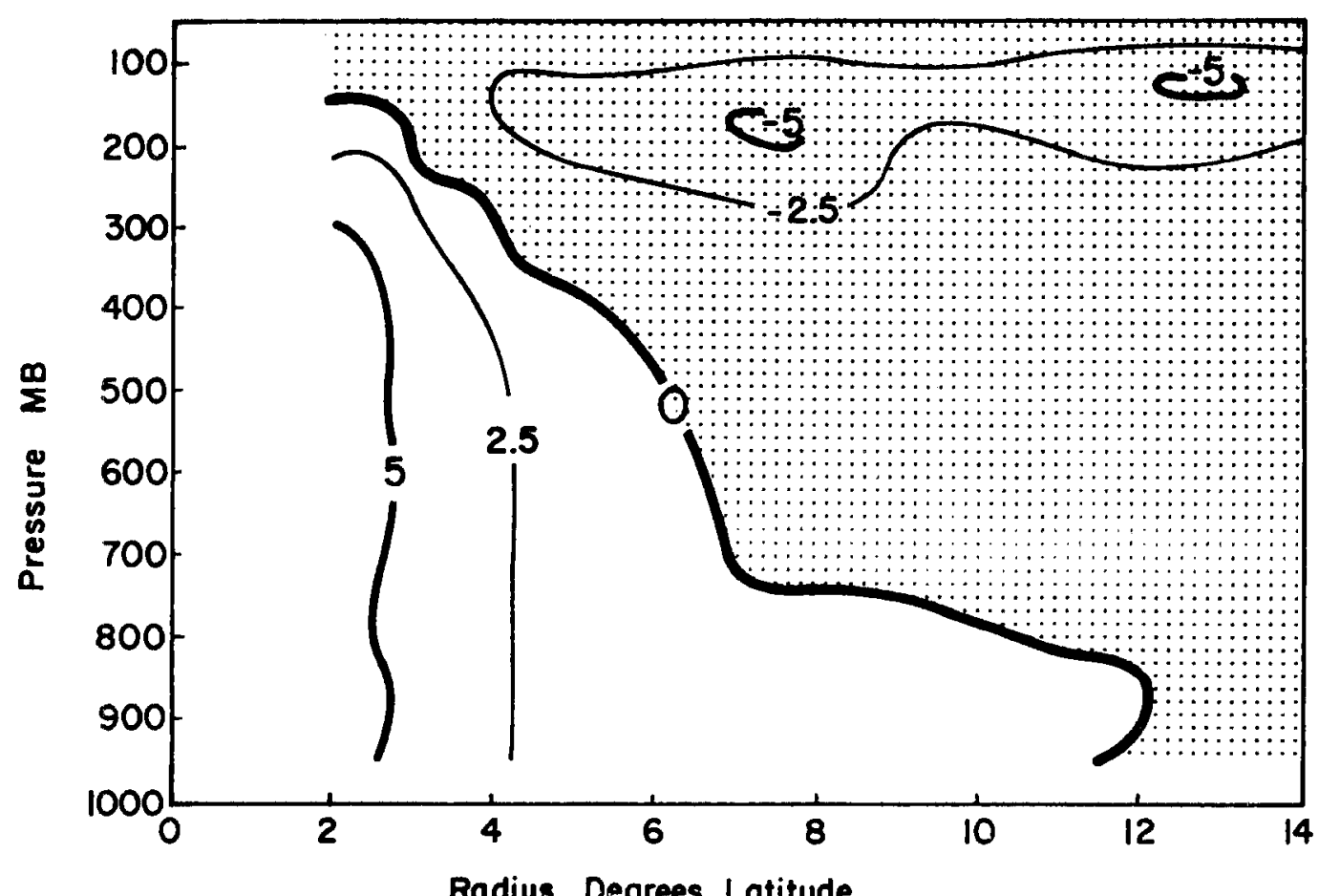

Fig. 34. Same as Fig. 29 except for Atlantic large hurricane north minus large tropical storm. 


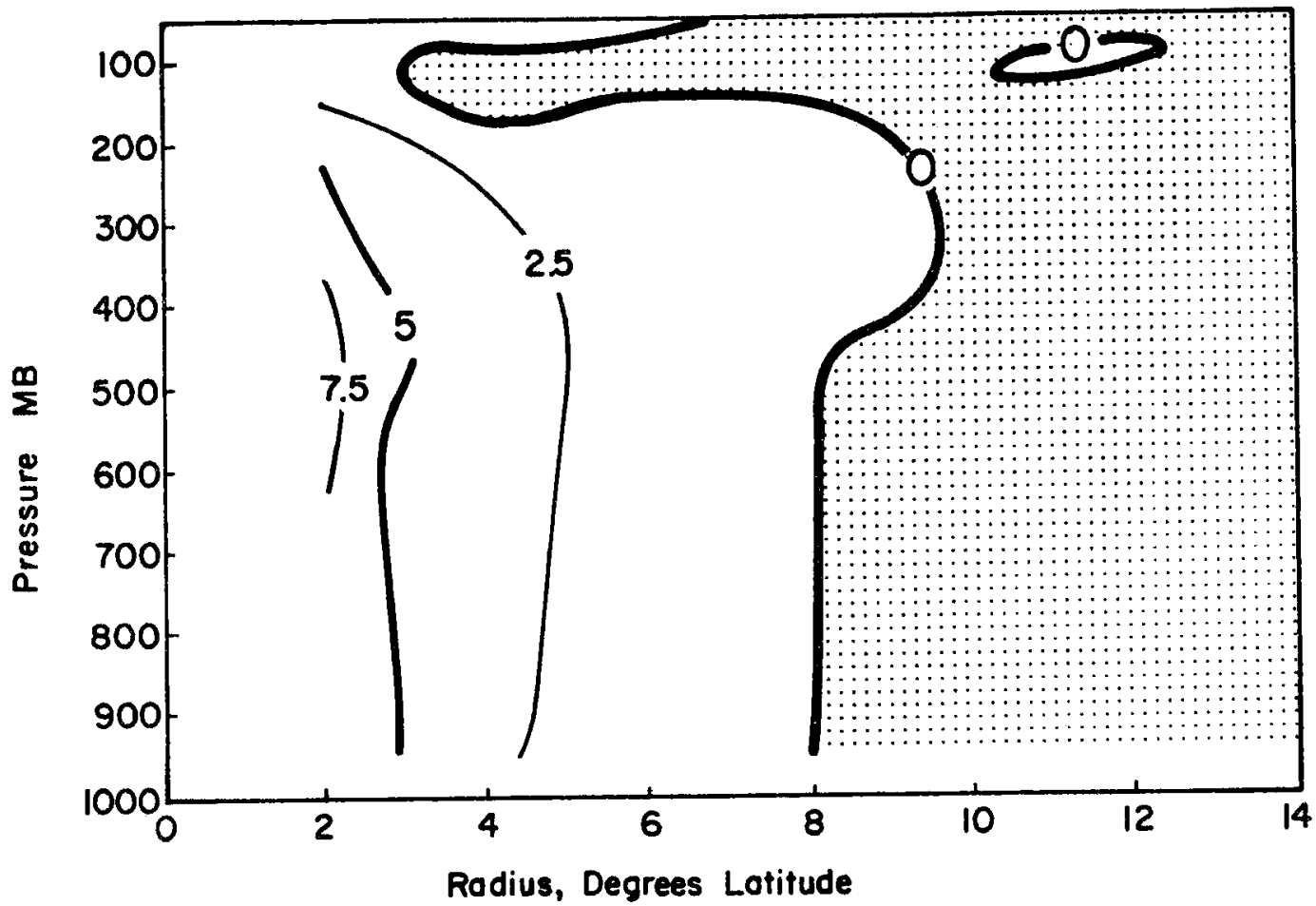

Fig. 35. Same as Fig. 29 except for Pacific sma11 typhoon minus sma11 tropical storm.

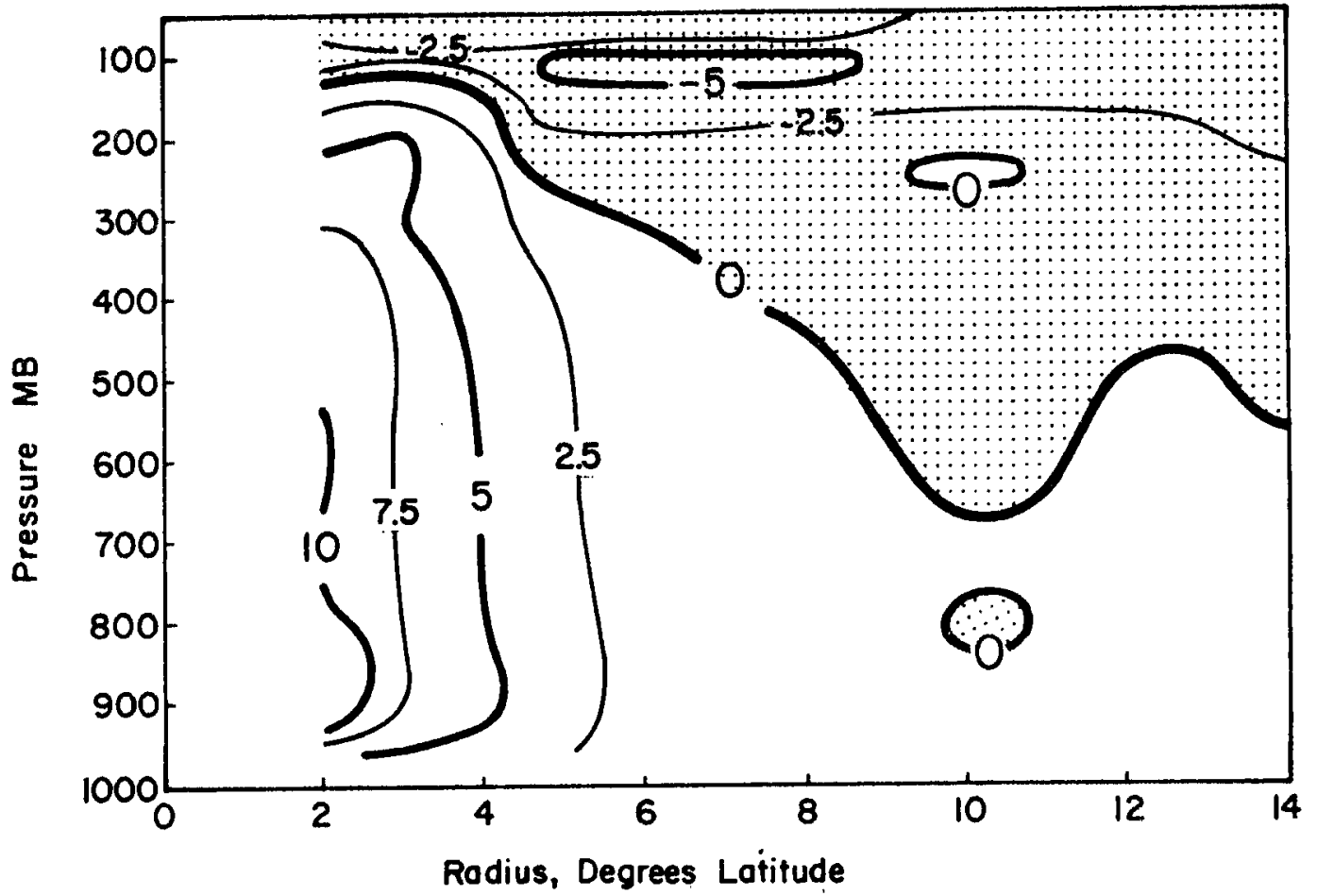

Fig. 36. Same as Fig. 29 except for Pacific large typhoon minus large tropical storm. 


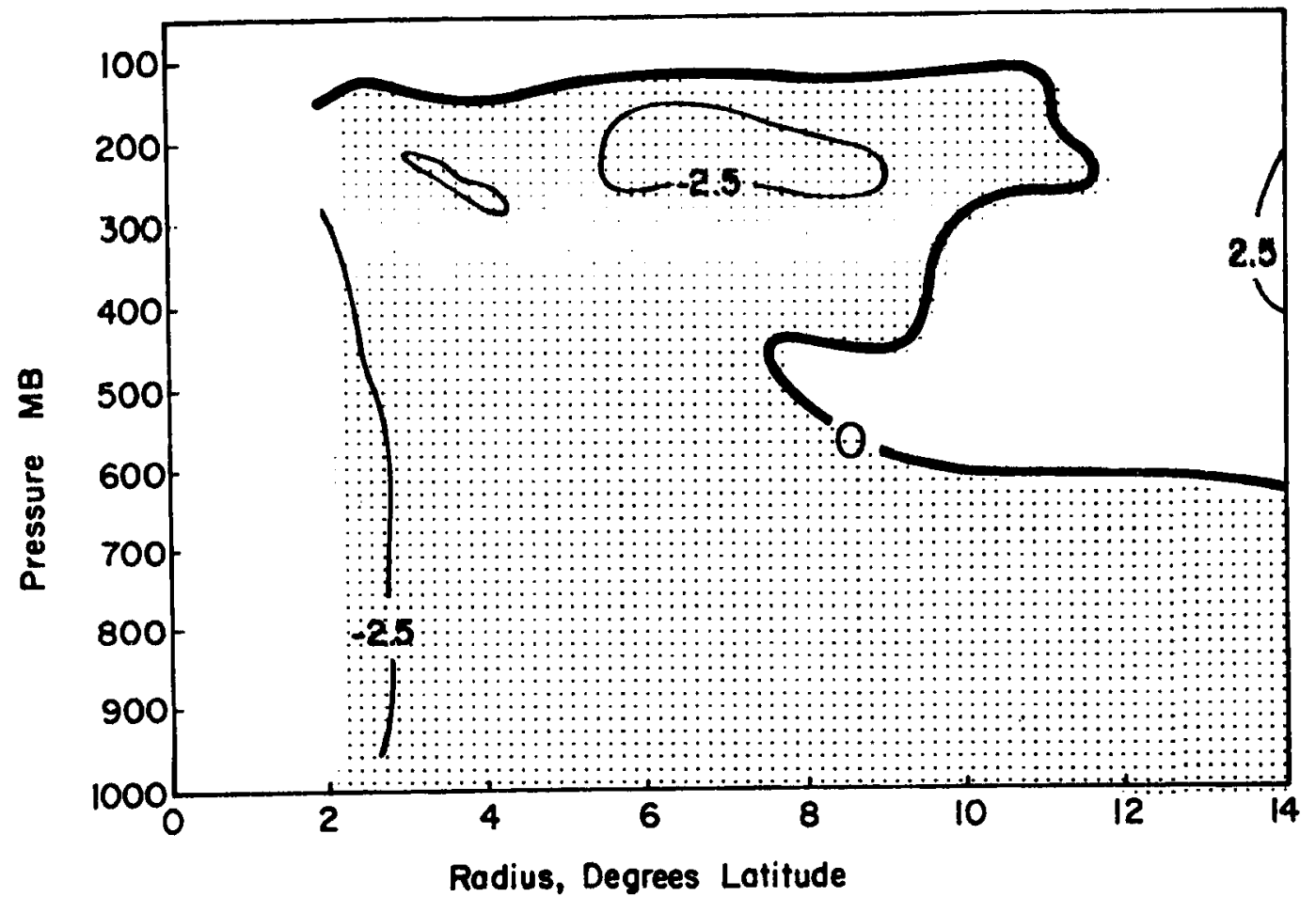

Fig. 37. Same as Fig. 29 except for Atlantic small/medium hurricane minus Pacific small typhoon.

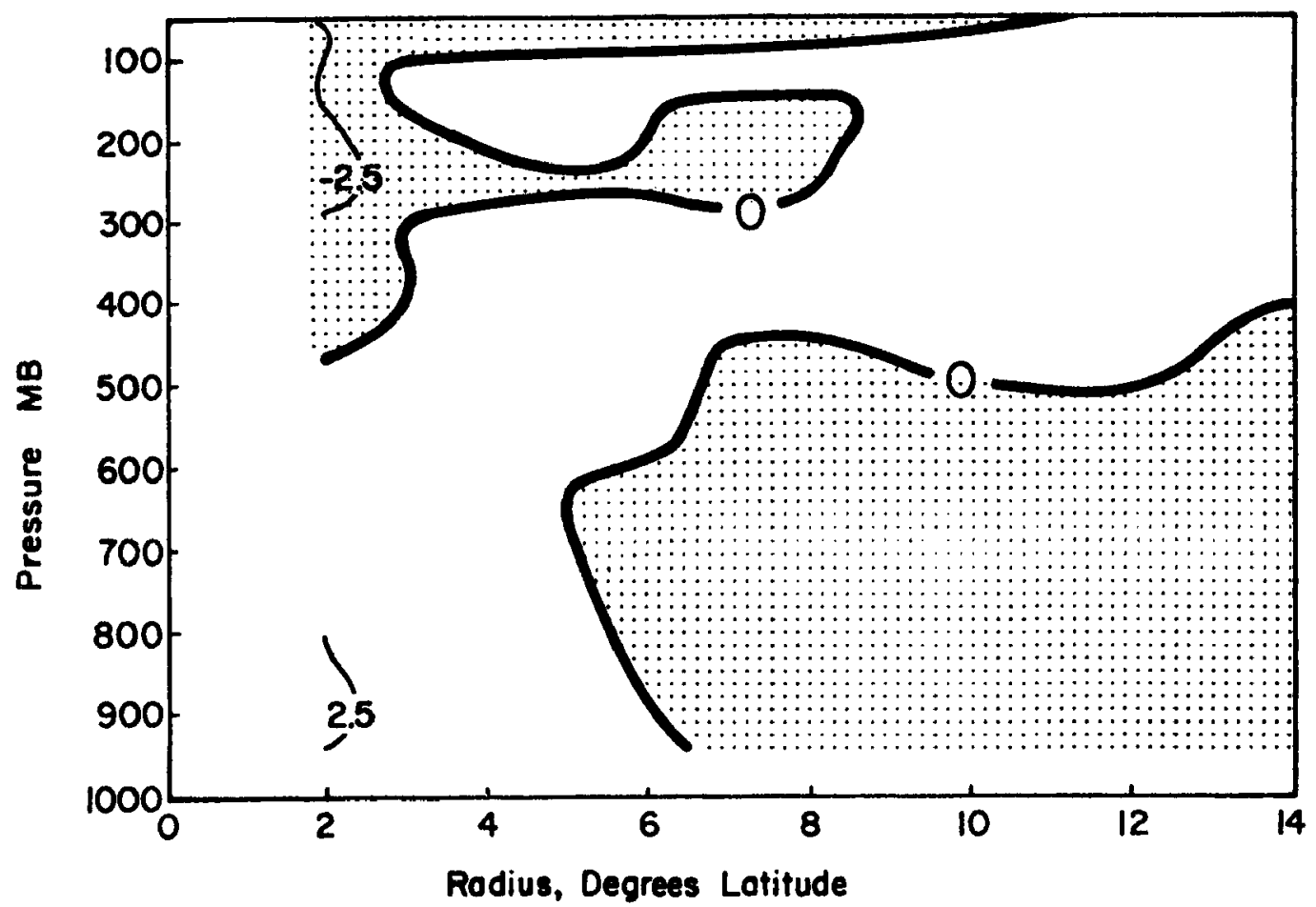

Fig. 38. Same as Fig. 29 except for Atlantic 1arge hurricane north minus Pacific medium typhoon. 
lesser intensity. Figure 38 compares the large hurricane north with a medium typhoon - again both systems are of equivalent size and the differences are mainly apparent inside $2^{0}$ radius.

\subsection{Statistical Significance of Tangential Wind Differences}

One criticism leveled at the rawinsonde compositing method in the past is that formal assessment of the statistical significance of the differences of means was seldom made. Because this paper uses an indirect definition (the outer closed isobar measurement) to estimate the size of the wind field, statistical support of the wind differences is even more important.

The $850 \mathrm{mb}$ winds are examined because they can be related to surface winds (Frank and Gray, 1980). From the $850 \mathrm{mb}$ wind distributions it is possible to make not only statistical calculations but also frequency-of-occurrence tables for surface wind speeds above a threshold valne.

The tangential winds at $850 \mathrm{mb}$ are tabulated for all 8 octants and 8 belts in the ROT coordinate system for each Atlantic composite. Because the rawinsonde stations are not evenly distributed, some of the octants in the rawinsonde composites have more observations than others. This complicates the statistical analysis. Adding all of the tangential wind observations together and dividing by the total to get a mean will give a biased result; the type of bias depends on where most of the observations were. If most of the observations were to the right of the cyclone (where the tangential wind is usually stronger) this mean wonld be larger than the mean obtained if the belt were sampled perfectly and the observations were thus evenly distributed over all octants. It is 
important that each octant, rather than each observation, is weighted equally. A method for combining, or 'pooling', the observations in this manner is described below.

The approach used is to weight individual observations more heavily in octants where they are less numerous. Wind data are tabulated into c1asses of $1 \mathrm{~m} \mathrm{~s}^{-1}$ width ( and belt in the composite grid (Fig. 28). The result is an array of frequencies $n_{i, j, k}$ each frequency is the number of observations in octant $i$ ( 1 through 8 ) and belt $j$ ( 1 through 8 ) which fell into speed c1ass k. For a particular belt and octant, adding all of the $n_{i, j, k}$ together gives the total number of observations in octant $i$ and belt $j$, designated by $M_{i, j}$. What is desired is a combination of all octant values of $n_{i, j, k}$ for a particulax be1t; the result $F_{j, k}$ is the pooled frequency of observations of wind class $k$ in belt $j$, and is given by Eq. 1 ,

$$
F_{j, k}=\sum_{i=1}^{O c t} n_{i, j, k} W_{i, j}
$$

where the weighting factor $W_{i, j}$ is given by

$$
W_{i, j}= \begin{cases}\sum_{i=1}^{8} M_{i, j} & M_{i, j} \neq 0 \\ \hline\left(M_{i, j}\right)(0 c t) & M_{i, j}=0 \\ 0 & \end{cases}
$$

where

$F_{j, k}=$ pooled frequency of $x \underline{t h}$ wind class in $j \underline{t h}$ belt 


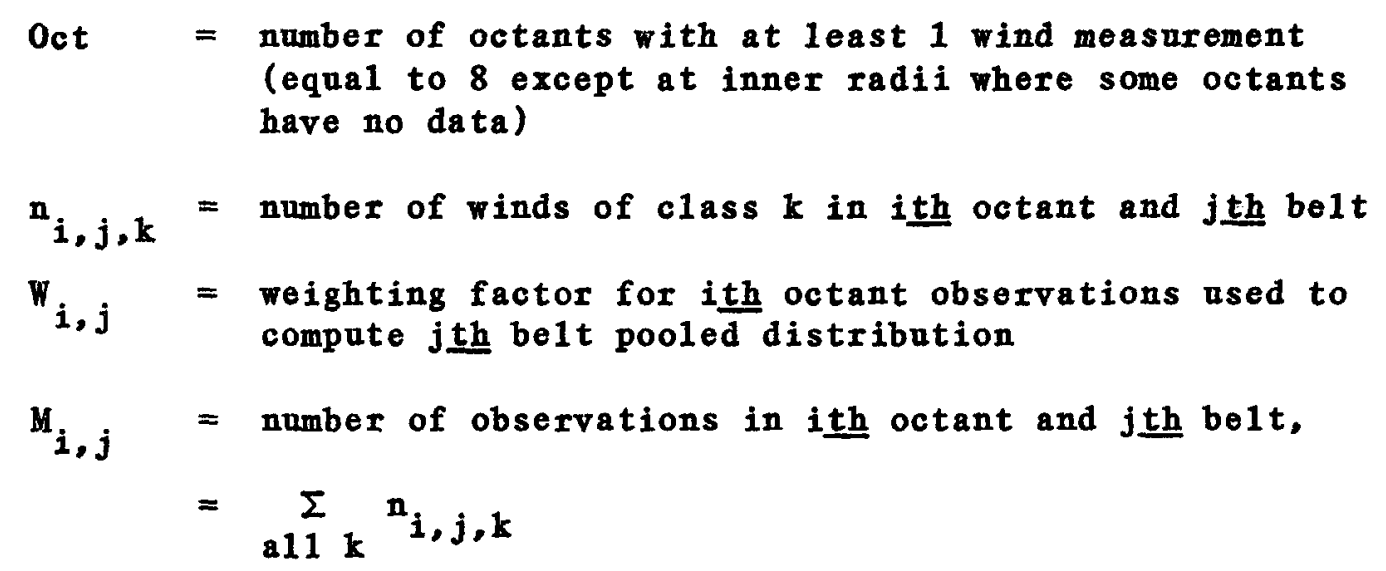

computed from the distribution of observations $M_{i, j}$ using Eq. 2 . Application of Eq. 1 to all wind classes (the weighting factors for a given octant and be1t need be computed only once at each 1evel) gives a frequency distribution of tangential wind for be1t $j$. The mean and standard deviation of this distribution can then be calculated in the usual way. Equation 3 gives an expression for the mean of the pooled distribution. This mean is the same as that obtained by averaging all of the octant means together,

$$
\bar{X}_{j \text { pooled }}=\frac{\sum_{a 11 k} F_{j, k} X_{k}}{\sum_{a 11 k} F_{j, k}}=\frac{1}{\operatorname{oct}} \bar{X}_{i, j}
$$

where

$$
\begin{aligned}
& \overrightarrow{\mathrm{x}}_{j \text { pooled }}=\text { mean of variable } x \text { for be1t } j \text {, based on the pooled } \\
& \text { distribution } \\
& F_{j, k} \quad=\text { pooled frequency of wind class } k \text { in belt } j \\
& X_{k} \quad=\begin{array}{l}
\text { midpoint of class } k \text { (i.e. the wind class } 6 \leq v<7 \text { would } \\
\text { have midpoint } 6.5 \text { ). }
\end{array}
\end{aligned}
$$


Figures 39 and 40 are prepared using tho pooled frequency

distributions of $850 \mathrm{mb}$ tangential wind for 7 belts (the $0-1^{\circ}$ latitude be1t is omitted because it contains so few observations). The means are computed using Eq. 3 , and the $95 \%$ confidence 1 imits for the means estimated by using the t-distribution and the standard deviations of the pooled distributions (Wa1pole and Myers, 1978). This test assumes that: 1) the observations making up the sample are independent and 2) the underlying population is normally distributed. Inspection of the distributions shows the second assumption to be quite good. The first is less accurate because some of the observations come from the same tropical cyclones. However, the differences between the means in Figs. 39 and 40 are so great that even using expanded confidence intervals to compensate for the dependence in the data would not affect the significance of the results.

4.4 Extent of $15 \mathrm{~m} \mathrm{~s}^{-1}(30 \mathrm{kt})$ Surface Winds

Cyclone size becomes an important practical consideration because of the extent of the surface wind field. Frank and Gray (1980) prepared a study of the frequency of occurrence of $15 \mathrm{~m} \mathrm{~s}^{-1}(30 \mathrm{kt})$ surface winds around tropical cyclones using rawinsonde compositing methods. They assumed the surface winds to be $75 \%$ of the $850 \mathrm{mb}$ winds, and tabulated the frequency of occurrence of $20 \mathrm{~m} \mathrm{~s}^{-1} 850 \mathrm{mb}$ winds around Atlantic cyclones of different intensities and Pacific cyclones of different intensities and sizes. They found that high winds at a given radius are more frequent for 1 arger and more intense cyclones, and relatively more frequent on the right side of the vortex than on the left. 


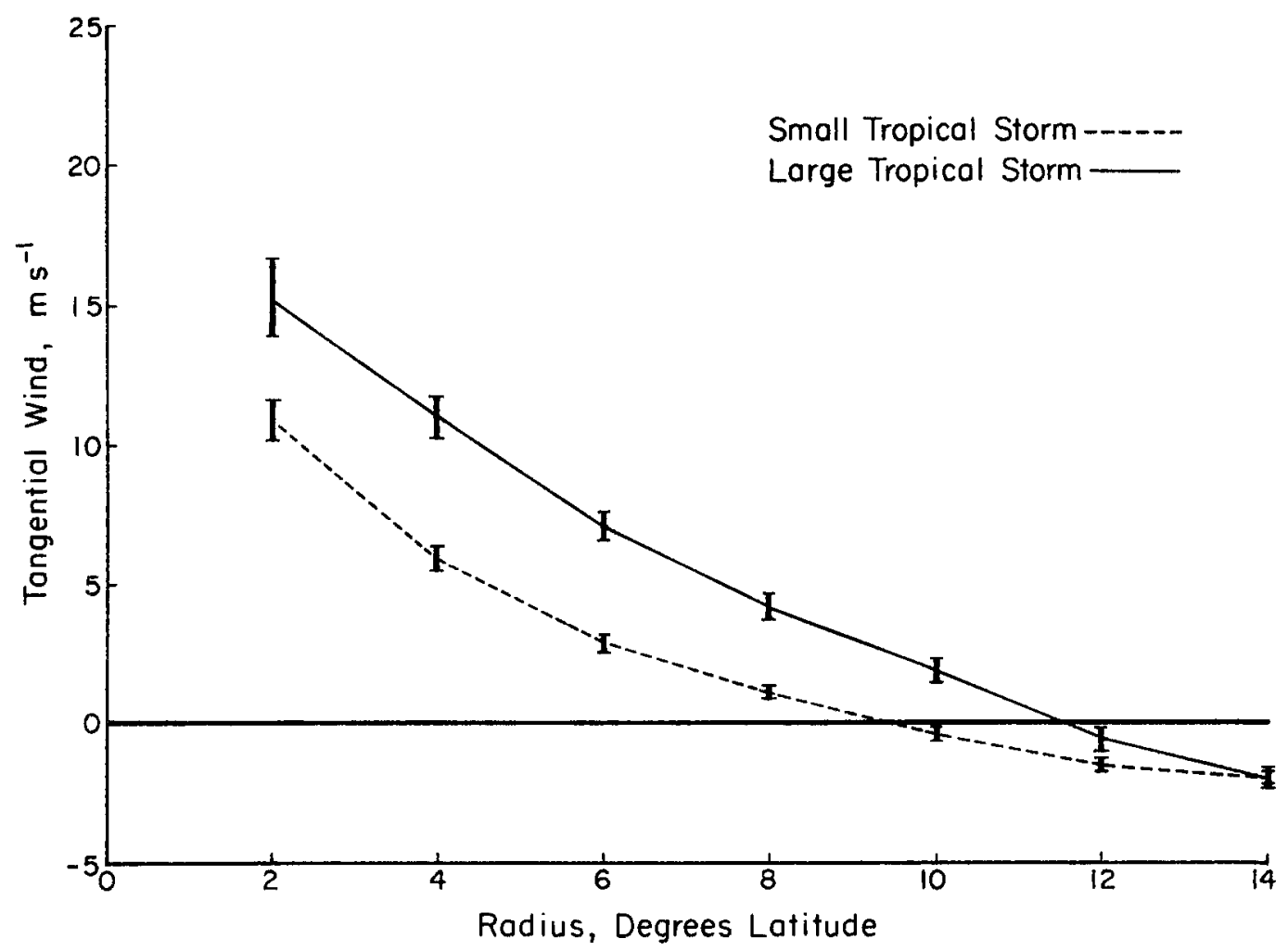

Fig. 39. Radial profile of azimuthal mean tangential wind for Atlantic tropical storms. Vertical bars denote the $95 \%$ confidence 1 imits on the mean at each radius.

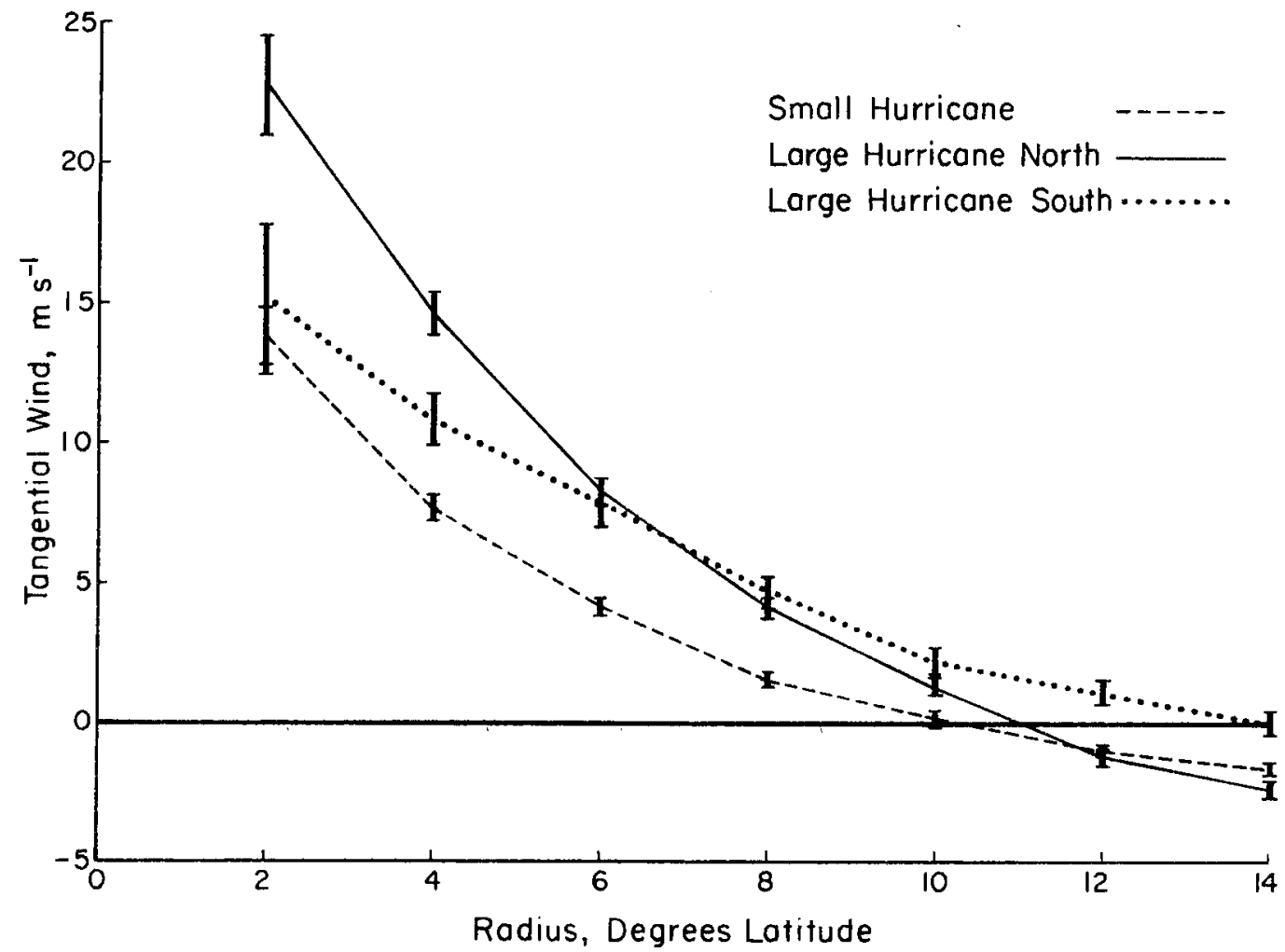

Fig. 40. Same as Fig. 39 except for Atlantic hurricanes. 
Using the wind speed frequency distributions from the Atlantic rawinsonde composites doscribod oarlier in this chapter, the froquenoios of $20 \mathrm{~m}^{-1} 850 \mathrm{mb}$ winds wore computed for $1-3^{\circ}, 3-5^{\circ}$, and $5-7^{\circ} 1 \mathrm{atitude}$ radius for each octant. The results are plotted in Figs. 41-44. No values are shown inside $1^{\circ}$ latitude where the number of observations is insufficient to obtain stable frequencies. The pattern which emerges is similar to that observed in the mean winds - high winds at a given radius are more frequent in large cyclones than sma11 ones.

These wind fields were obtained from composites, but show the probability of encountering $15 \mathrm{~m} \mathrm{~s}^{-1}$ surface winds at various locations around individual cyclones of different sizes and strengths. With refinements such frequencies could aid forecasters in estimating the extent of gale force winds when central pressure and radius of outerclosed-isobar estimates are available. By compositing satellite winds and surface reports from ships, it might be possible to obtain a more detailed and finely stratified set of figures like these which would be of real value in forecasting and ship routing. 


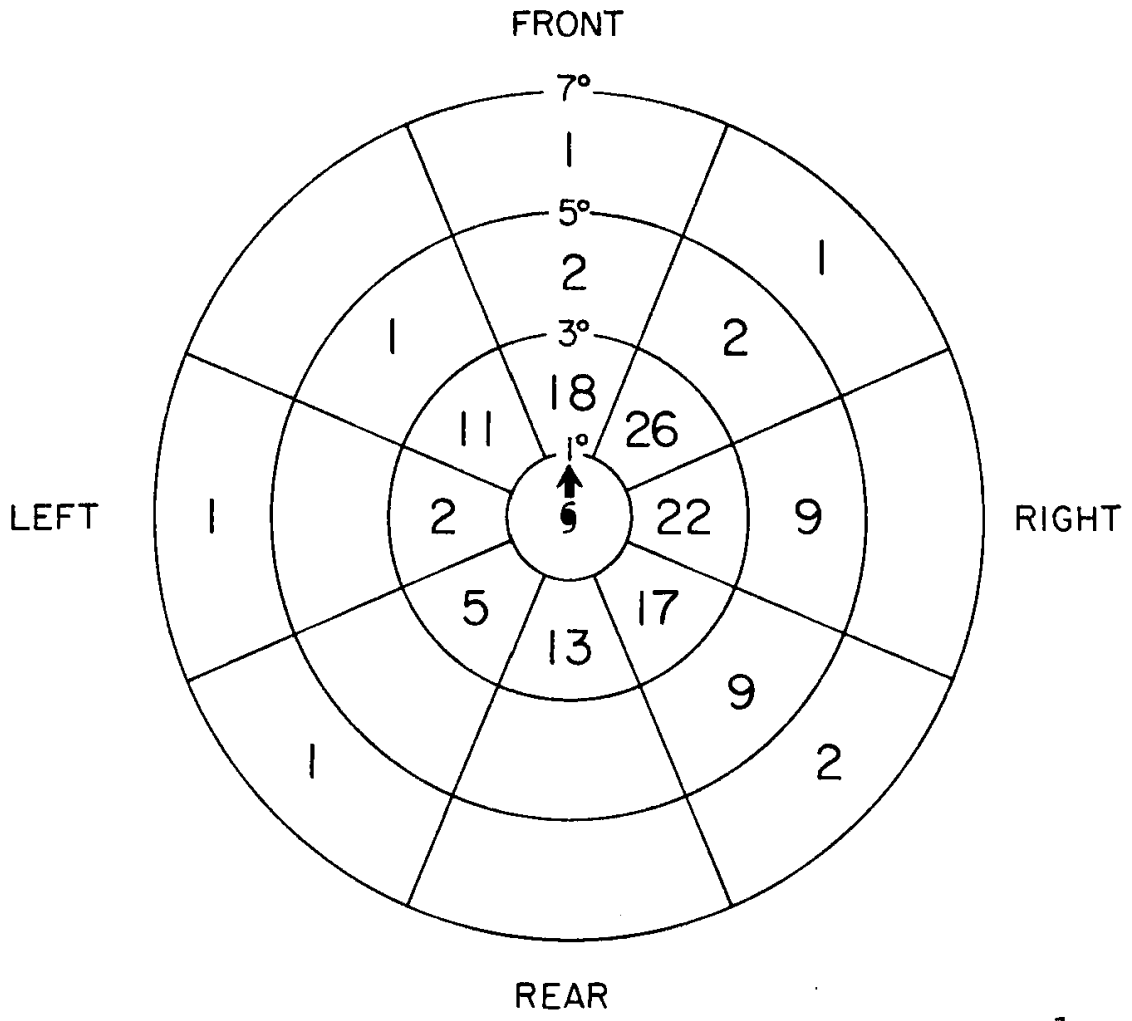

Fig. 41. Percent frequency of $850 \mathrm{mb}$ wind speeds of $20 \mathrm{~m} \mathrm{~s} \mathrm{~s}^{-1}$ or more (approximately $15 \mathrm{~m} \mathrm{~s}^{-1}$ at the surface) around small tropical storms in the Atlantic.

FRONT

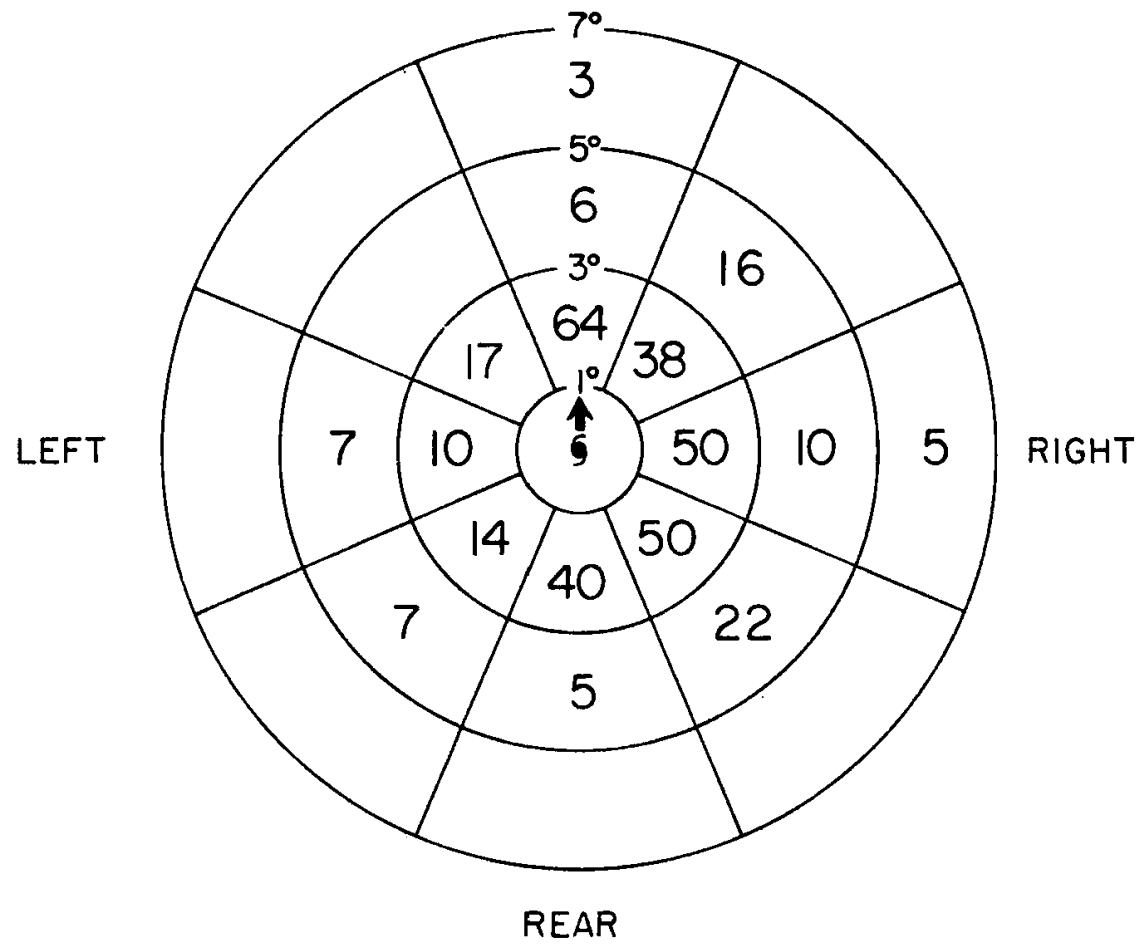

Fig. 42. Same as Fig. 41 except for large tropical storms. 


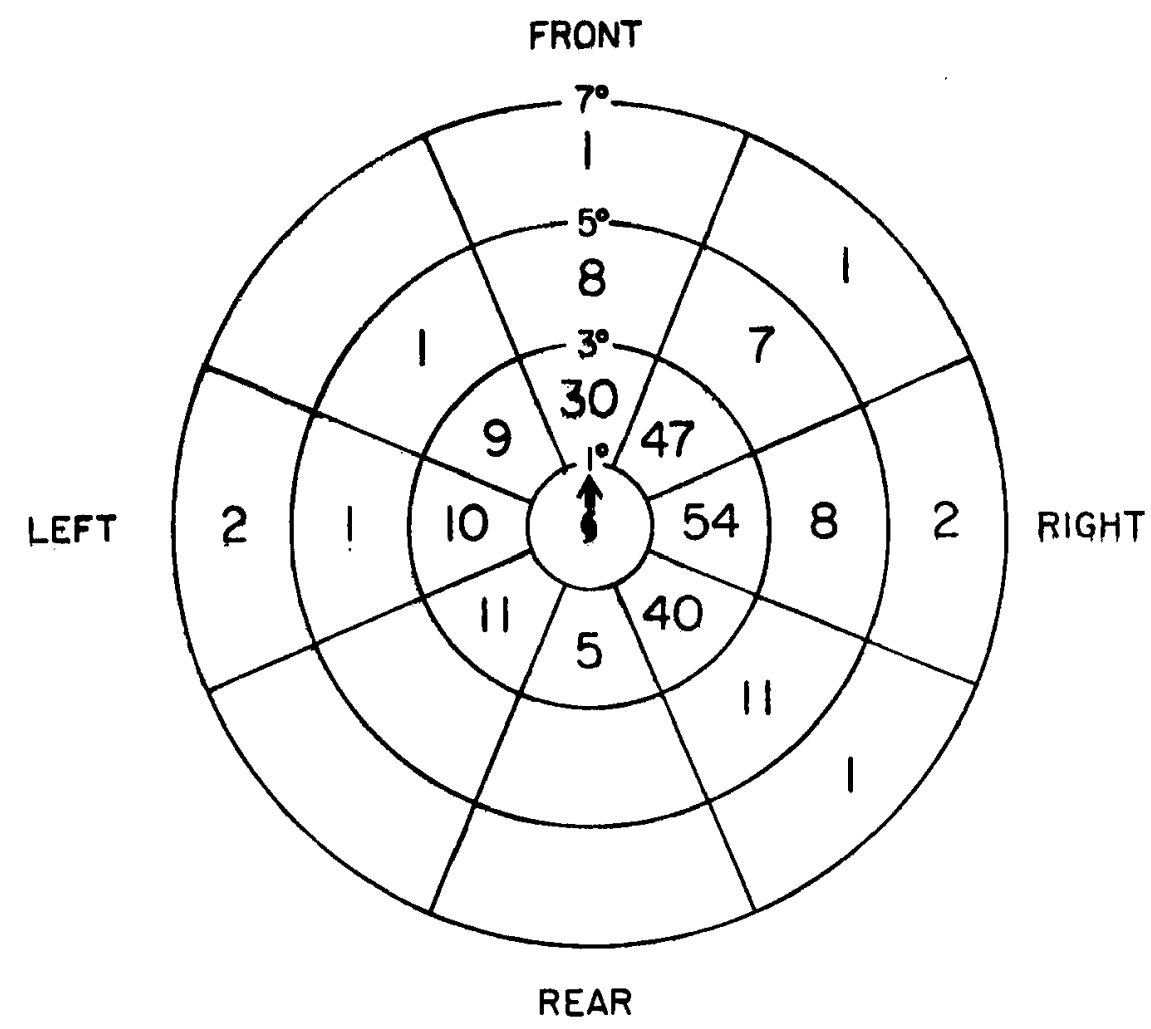

Fig. 43. Same as Fig. 41 except for small hurricanes. FRONT

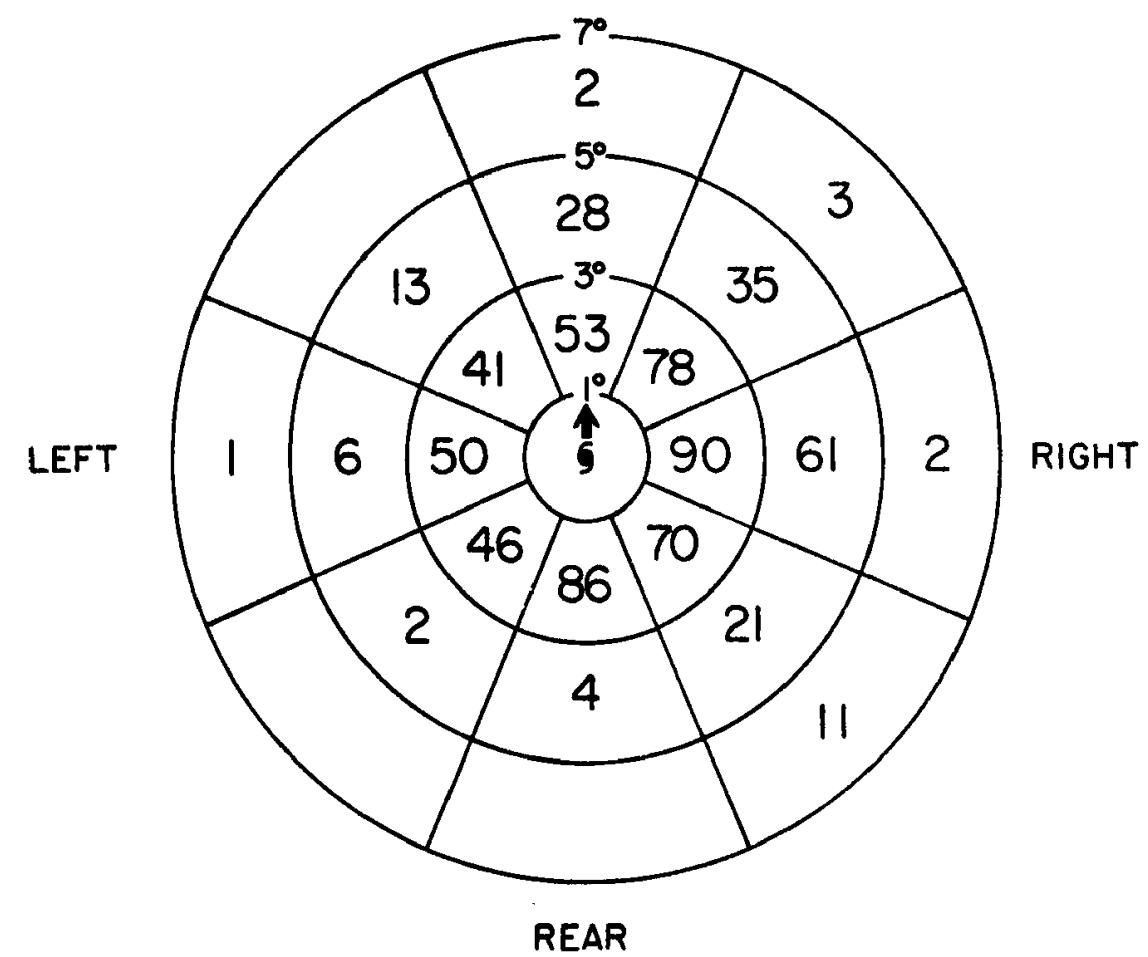

Fig. 44. Same as Fig. 41 except for large hurricanes north of $25^{\circ}$ latitude. 
5. MAINTENANCE OF LARGE AND SMALL CYCLONES AND ANGULAR MOMENTUM CONSIDERATIONS

\subsection{Introduction}

In the previous two chapters it has been demonstrated that tropical cyclones exhibit a range of sizes as well as maximum winds, and that different sizes of cyclones tend to occur during particular times of yoar and in particular locations. Significant structural differences in both wind and mass fields are also apparent - large cyclones logically perturb the basic state of the atmosphere over a larger area than do sma11er ones. This chapter discusses the implications of these climatological and structural findings and attompts to provido a physical explanation for the observed characteristics of tropical cyclone size.

5.2 A Discussion of Angular Momentum in Tropical Cyclonos

A powerful constraint on the tropical cyclone is the conservation of angular momentum. The angular momentum about the cyclone center is given by

$$
\begin{aligned}
\mathrm{AAM} & =\mathrm{EAM}+\mathrm{RAM} \\
& =1 / 2 \mathrm{f}_{0} \mathrm{x}^{2}+\mathrm{IV}
\end{aligned}
$$

where $r$ is radius from the center, $f_{0}$ is the Coriolis parameter at the latitude of the cyclone center, and $v$ is the tangential wind. The absolute angular momentum (AAM) is the sum of earth angular momentum (EAM) and zelative angular momentum (RAM). The tropical cyclone, 1ike 
any synoptic storm, is a concentration of cyclonic RAM which must be accumulated and maintained in order for the storm to develop and persist. This concentration is brought about by a circulation which imports angular momentum. Pfeffer (1958), and Holland (1982) havo studied the angular momentum fluxes of tropical cyclones and have found that the mean transverse circulation dominates within $400 \mathrm{~km}$ of the center. Net inflow occurs in the low and middle troposphere where the tangential wind is strong and cyclonic (high RAM), and net outflow occurs in the upper troposphere, with 10w RAM. The result is a net import of cyclonic RAM into the cyclone. At radii beyond $400 \mathrm{~km}$, the momentum transports are increasingly the result of horizontal eddy fluxes, particularly in the upper troposphere where large asymetries are often observed (B1ack and Anthes, 1971; Lee, 1982).

For the tropical cyclone to remain in steady state, it is necessary that the angular momentum flux into the cyclone offset the surface 1osses. If the cyclone is also moving poleward (as is usually the case), greater imports of RAM are required to account for the conversion of RAM to EAM (Coriolis torque). Observations show the lattor requirement to be several times larger than the former outside the high-energy core of the cyclone (Ho1land, 1982).

An increase in momentum influx beyond the transports needed to balance friction and EAM changes will increase the RAM of the cyclone. One result would be an increase in lower-tropospheric surface wind and hence surface stress. In time, the increase in surface losses of RAM would equal the increased transports and the cyclone would again approach steady state. What is not immediately clear is how the increase in RAM will be distributed - it could go to increase the 
maximum winds (intensification), the average wind speed in the vortex (strength), or the extent of the circulation (growth). In the next section it will be demonstrated that the high-energy core is relatively unimportant to the total angular momentum of the tropical cyclone, while the winds at greater radii are crucial. It is therefore hypothesized that an increased convergence of angular momentum into a tropical cyclone is directly associated with growth and strengthening, but not necessarily with cyclone intensification.

5.3 Observed Angular Momentum Associated with Large and Sma11 Tropical Cyclones

Using the rawinsonde composites described in Chapter 4, the values of relative angular momentum, earth angular momentum, absolute angular momentum, and loss of angular momentum due to surface stress were calculated for $1^{\circ}$ latitude radial belts from $2^{\circ}$ to $6^{\circ}$ and $2^{\circ}$ belts from $6^{0}$ to $14^{\circ}$, from $950 \mathrm{mb}$ to $100 \mathrm{mb}$.

Because few rawinsonde observations exist inside $2^{\circ}$ radius, it was necessary to obtain an approximation to the wind field. It is assumed that the vertically integrated wind field can be approximated by a modified Rankine vortex (Hughes, 1952) of the form

$$
v= \begin{cases}v_{0}\left(\frac{v_{0}}{v_{\max }}\right)^{-1-\frac{1}{x}} \frac{r}{r_{0}} & 0 \leq x \leq r_{\max } \\ v_{0}\left(\frac{r_{0}}{r}\right)^{x} & r_{\max } \leq r\end{cases}
$$


where

$\mathrm{V}=$ tangential wind,

$v_{0}=$ tangential wind at specified radius $x_{0}$,

$v_{\max }=$ maximum wind in the vortex,

$x=$ empirical vortex parameter, usually in the range 0.4 to 0.6 . It is here set equal to 0.5 ,

$\mathbf{r}=$ radius,

$r_{0}=$ radius of $v_{0}$, here taken to be $2^{0} 1$ atitude or $222 \mathrm{~km}$, and

$r_{\max }=$ radius of maximum winds.

Such a profile has been demonstrated to fit surface winds with

reasonable accuracy (Rieh1 and Ma1kus, 1960) with va1ue of $x=0.5$.

Shea and Gray (1973) indicate that the value of $x$ in the upper

troposphere is closer to 0.75. However, Shea and Gray (1973) also shows

that the shape of the tangential wind profile in the high-energy area

near the center changes little up to $500 \mathrm{mb}$, so for this calculation a

value of 0.5 will be used.

This profile is used to estimate the radial dependence of the

vertically averaged wind inside $2^{\circ}$ radius, using the following steps:

1) Estimate $V_{\max }$ using the average maximum winds of the cyclones for which the composite was made.

2) Subtract the mean cyclone speed of motion from the maximum wind to obtain an estimate of the azimuthal average maximum wind.

3) Estimate the surface $V_{0}$ for $r_{0}$ at $2^{\circ}$ latitude radius using $75 \%$ of the $850 \mathrm{mb}$ composite tangential wind at $2^{\circ}$.

4) Solve Eq. 5 for $r_{\max }$, using $x=0.5$. 
5) Use $r_{\max }$ obtained in Eq. 4 and assume that $r_{\max }$ is constant with height (Shea and Gray, 1973) to obtain vertically averaged $I_{\text {max }}$

6) Calculate vertically integrated $v_{0}$ from composite tangential winds at $2^{\circ}$ latitudo radius.

7) Uso $v_{0}$ and $r_{\max }$ to solvo Eq. 5 for estimated vertically averaged $V_{\max }$.

This fitted vortex can be multiplied by $I$ and integrated exactly to yield an estimate of the RAM from the center of the cyclone to $2^{\circ}$ latitude radius. Al though the assumed profile shape and estimated $\mathbf{r}_{\mathrm{mx}}$ for the tropospheric mean vortex are subject to exror, an examination of Figs. 45 and 46 show the angular momentum of the $0-2^{\circ}$ region to be small when compared with that of the rest of the circulation.

The $0-4^{\circ}$ momentum is related to both cyclone size and intensity. The Paoific typhoons have moro momontum than Pacific tropical storms, but within each intensity class the momentum content increases monotonically with size. The Atlantic is less consistent, as the large tropical storm has more momentum than the small hurricane, but the dual influence of size and intensity is still apparent. The large hurricane south of $25 \mathrm{~N}$ is a bit unusual - observations of its relatively low tangential winds at $2^{\circ}$ and strong climatological maximum wind of $45 \mathrm{~m}$ $s^{-1}$ (highest of the Atlantic sets) place it as a relatively 'tight' intensifying vortex within a large circulation envelope.

Figure 46 shows the integrated momentum from the center to $8^{\circ}$. Here, the effect of the assumed Rankine profile from $0-2^{\circ}$ (shown in 


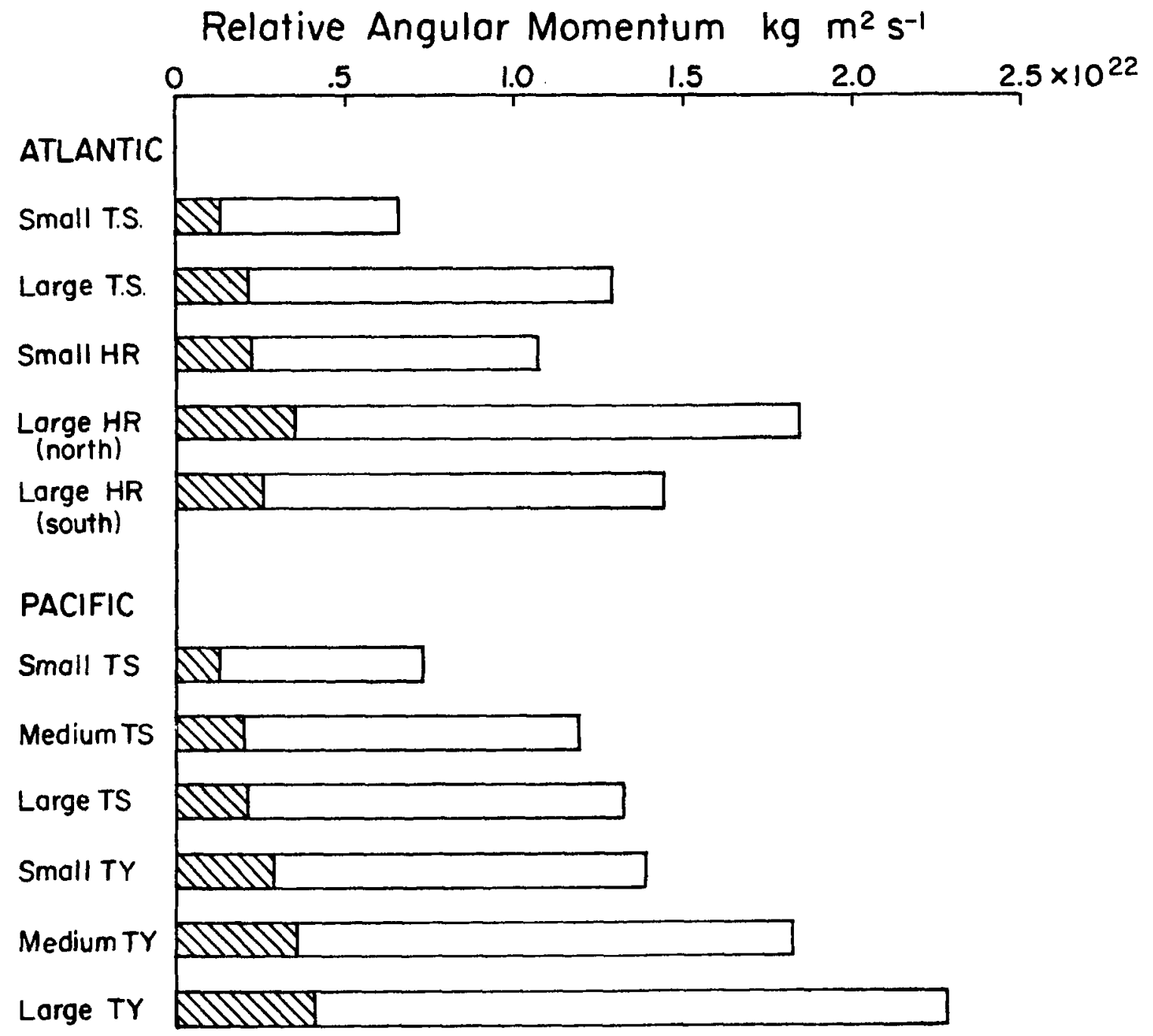

Fig. 45. Integrated relative angular momentum (RAM) from $0^{\circ}-4^{\circ}$ atitude radius and $950 \mathrm{mb}$ to $100 \mathrm{mb}$ for Atlantic and Pacific tropical cyclone composites. The shaded area is the RAM estimated for the $0^{\circ}-2$ region using the fitted modified Rankine vortex approximation described in the text. 


\section{Relative Angular Momentum $\mathrm{kg} \mathrm{m}^{2} \mathrm{~s}^{-1}$}

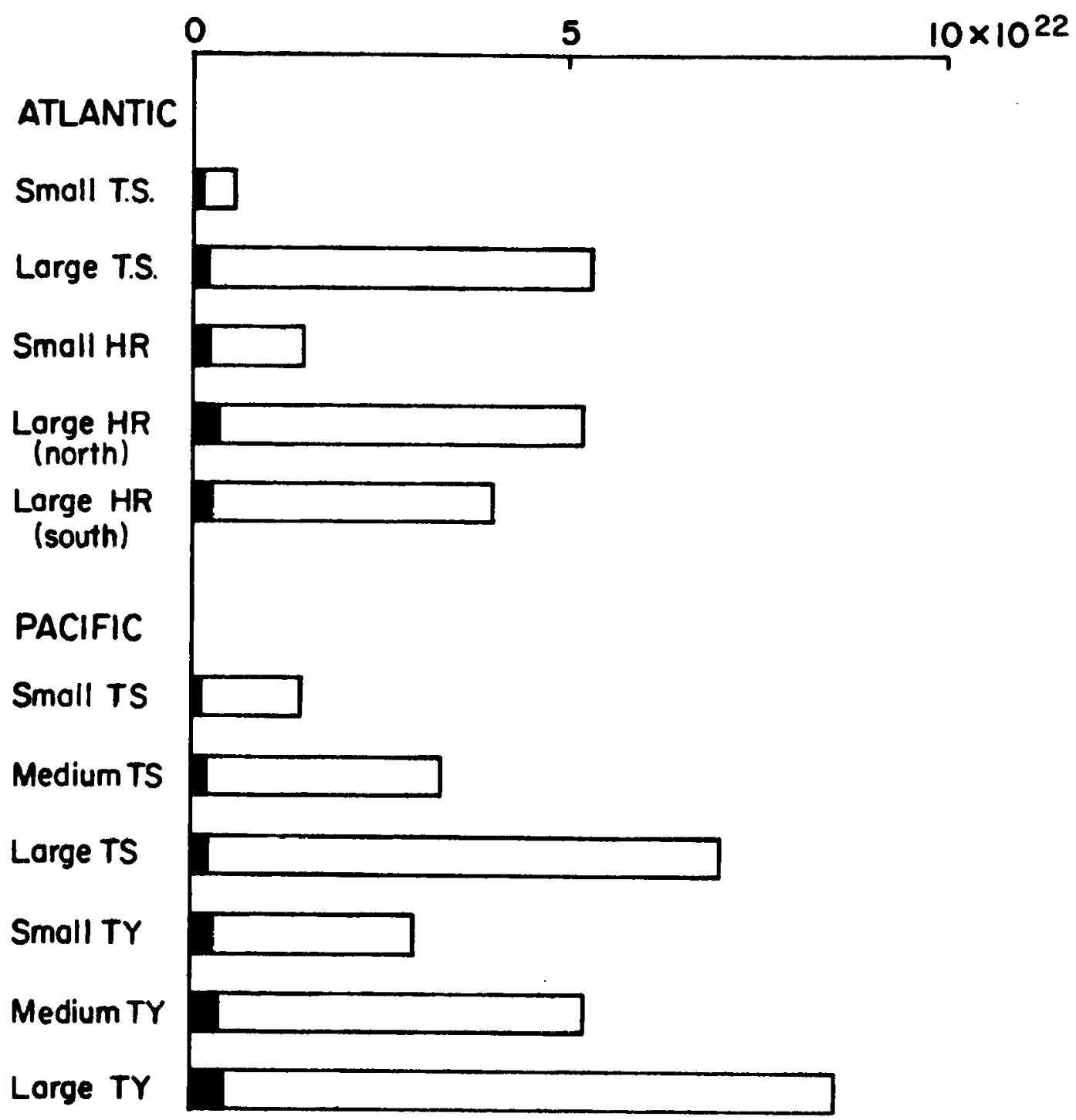

Fig. 46. Same as Fig. 45 except from $0^{\circ}-8^{\circ}$ radius. Shaded area is again the estimated RAM from $0^{\circ}-2^{\circ}$ latitude radius. 
b1ack) is negligible, and size is clearly dominant in determining momentum amount. The large tropical storms contain more momentum than a11 but the large hurricanes and typhoons, and only within a given size class do intense systems necessarily possess more momentum than weaker ones.

Figure 47 shows surface losses of angular momentum, obtained by numerica11y integrating

$$
F=\int_{0}^{2 \pi} 0^{\circ} \int_{1 a t}^{\circ} \rho r C_{D}\left|v_{s f c}\right| v_{s f c} r d r d \theta
$$

where $C_{D}=7.5 \times 10^{-4}+6.7 \times 10^{-5} \mathrm{lv}_{\text {sfc }} \mid\left(v_{\text {sfc }}\right.$ in m $\mathrm{s}^{-1}$ ) (Garratt, 1977; Ho1land, 1982) using $75 \%$ of the $850 \mathrm{mb}$ composite winds as an estimate of surface winds from $2^{\circ}$ to $8^{\circ}$ radius, and using the fitted Rankine vortex described earlier to estimate the surface winds from $2^{\circ}$ radius to the center. Surface stress follows a trend similar to that of RAM - a function of intensity and size at $0-4^{\circ}$, and more near1y a function of size alone at $0-8^{\circ}$. Even at $4^{\circ}$, the effects of size are plain - the larger systems are experiencing more surface stress than their smaller counterparts of equal intensity.

\subsection{Implications of Angular Momentum Constraints for Cyclone Size and Growth}

As discussed in the previous section, large tropical cyclones are observed to have more angular momentum than small ones and, over areas of $4^{\circ}$ radius and beyond, size is a more important factor than intensity in determining total cyclone RAM. Surface stresses al so follow the same trend - over areas of $8^{a}$ radius, size is an important control. Several implications arise from these observations. 


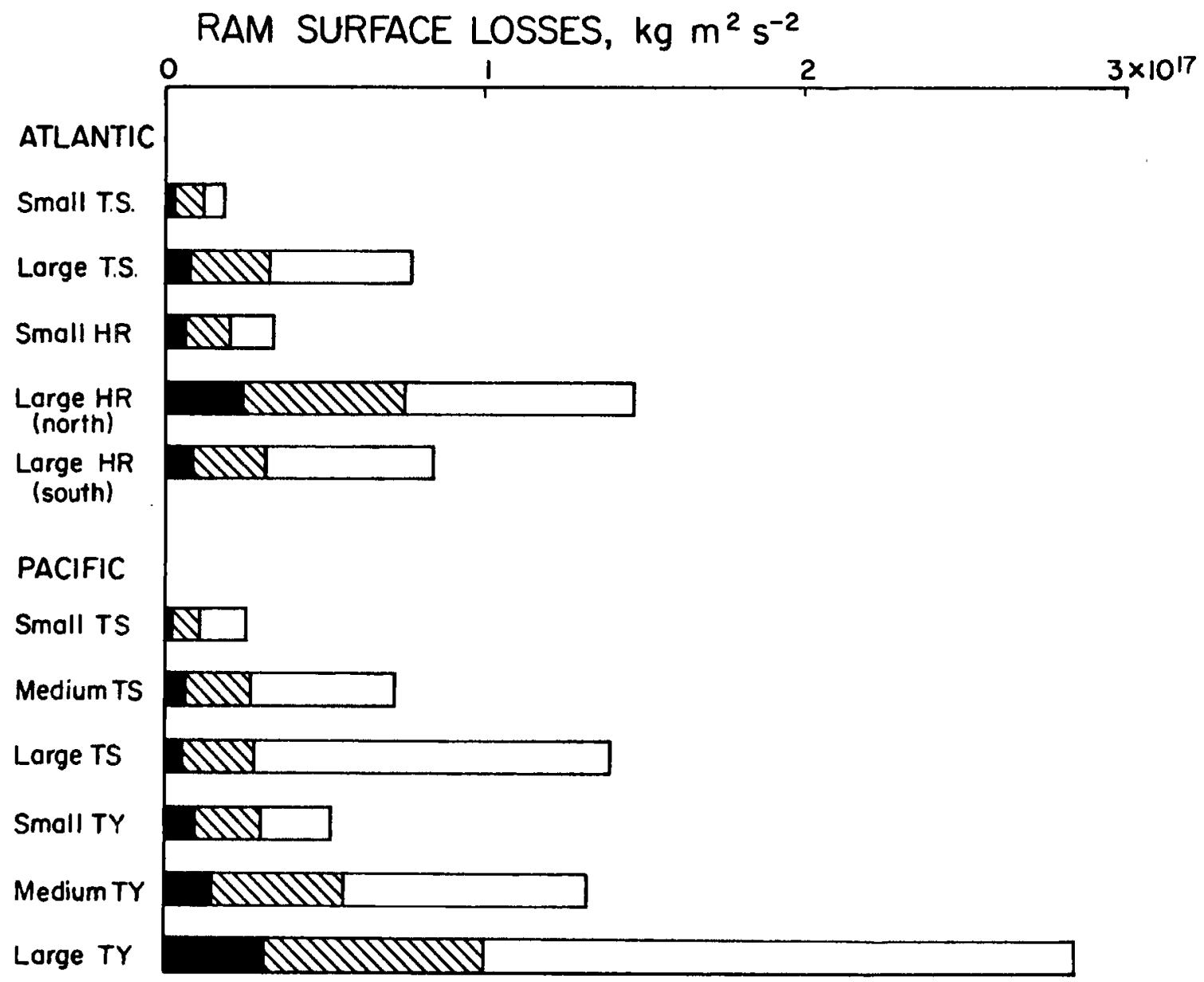

Fig. 47. Integrated surface sink of cyclone RAM from $0^{\circ}-8^{\circ}$ latitude radius fo Atlantic and Pacific tropical cyclone composites. The black area corresponds to surface losses in the area within $2^{2}$ latitude radius of the center, the hatched area from $2^{\circ}$ to $4^{\circ}$, and the remainder from $4^{\circ}$ to $8^{\circ}$. 
The most obvious implication is that the momentum transports into a large cyclone must be greater than those into a sma11 cyclone, just to maintain it in steady state. The ratio involved is quite large; as can be seen in Fig. 45 the large typhoon is subject to over five times as much surface stress as a sma11 one. The transports required for a growing, moving storm must be larger still. Suppose that a sma11 hurricane were to increase its ROCI by $2^{\circ}$ in $36 \mathrm{~h}$, becoming a medium hurricane, while moving northward from $20^{\circ} \mathrm{N}$ to $22.5^{\circ} \mathrm{N}$ (an average speed of $2.1 \mathrm{~m} \mathrm{~s}^{-1}$, and intensifying and strengthening such that the average wind inside $2^{\circ}$ latitude (hence the RAM a1so) were to increase to 1.5 times the 'small hurricane' estimate. The magnitude of the angular momentum transports required are shown in Table 6.

\section{TABLE 6}

Angu1ar momentum transports across $8^{\circ}$ radius required by a growing, intensifying cyclone.

PROCESSES

$$
\mathrm{kg} \mathrm{m}^{2} \mathrm{~s}^{-2}
$$

GROWTH

POLEWARD MOTION

SURFACE STRESS

INTENSIFICATION

AND STRENGTHENING

\section{IMPORT TO CYCLONE}

$$
\text { m } s^{-1} d^{-1} *
$$

\section{Percent}

$$
\begin{array}{r}
2.86 \times 10^{17} \\
1.94 \times 10^{17} \\
.90 \times 10^{17} \\
.08 \times 10^{17}
\end{array}
$$

$$
1.9
$$$$
1.3
$$$$
0.6
$$$$
49.5
$$$$
33.5
$$$$
15.5
$$$$
0.1
$$

*Angular momentum transport expressed as an average increase in tangential wind.

The requirement for growth was described earlier - the tangential wind is increasing at all radii. The 'motion' requirement arises because the system is moving poleward to higher values of the Coriolis parameter and the associated EAM is rising. Under conservation of angular momentum, this too must be accounted for, either by an 
equivalent decrease in RAM (which we have specified does not happen) or by transports. Note that the requirement to offset surface losses is sma11er than the first two, but of the same order, but the requirement for intensification is negligible.

What happens in nature, of course, is that the environment supplies a quantity of angular momentum and the cyclone responds, rather than the cyclone issuing 'requirements' as in the simple example presented above. A portion of the momentum goes to offset EAM changes as the cyclone moves in response to its environmental flow, and the remainder goes to spin up the wind field.

Growth of a tropical cyclone is hypothesized to result from increasing fluxes of angular momentum at large radii around the vortex. Holland (1982) has derived an equation for the tendency of relative angular momentum in Lagrangian (following the cyclone) coordinates and evaluated the terms using rawinsonde composite data. At large radii, the momentum budget is controlled mainly by three effects: 1) the meridional motion torque, 2) the eddy Coriolis torque and 3) the horizontal eddy RAM flux.

The meridional motion torque arises from the changing value of $f$ with latitude, as described previously. Poleward-moving cyclones experience an anticyclonic torque. The eddy Coriolis torque arises from the relative motion of the environmental flow and the cyclone. If air is flowing through the cyclone from pole to equator, it has a higher value of $f$ when entering than when leaving, and the cyclone experiences a cyclonic torque. Note that the meridional motion and eddy Coriolis torques work similarly, though they of ten oppose each other, as cyclones usually move poleward faster than the environmental flow (Chan and Gray, 
1982). The horizontal eddy RAM flux arises when inflow (outflow) in Lagrangian coordinates (cyclone motion vector subtracted from the winds) coincides with maximum cyclonic (anticyclonic) circulation.

With a growing cyclone we would expect more cyclonic values of these effects. A paradox is that growth is associated with recurvature and poleward motion - an increased anticyclonic motion torque. This is apparently offset by the other effects, however. Figure 48 shows two characteristic lower-tropospheric flows - one associated with a small cyclone and the other with a large or growing one. The presence of an anticyclone (often of polar origin) to the northwest of the growing cyclone results in an increased eddy Coriolis torque in the lower troposphere, and 1ikely an increased eddy RAM f1ux as well. Because the anticyclone is of polar origin, the meridional flow to the west of the cyclone is vertically sheared with southerlies overlying northerlies. The mean tropospheric wind is still souther1y, so the cyclone moves toward the north despite the opposing flow in the lowest levels. 

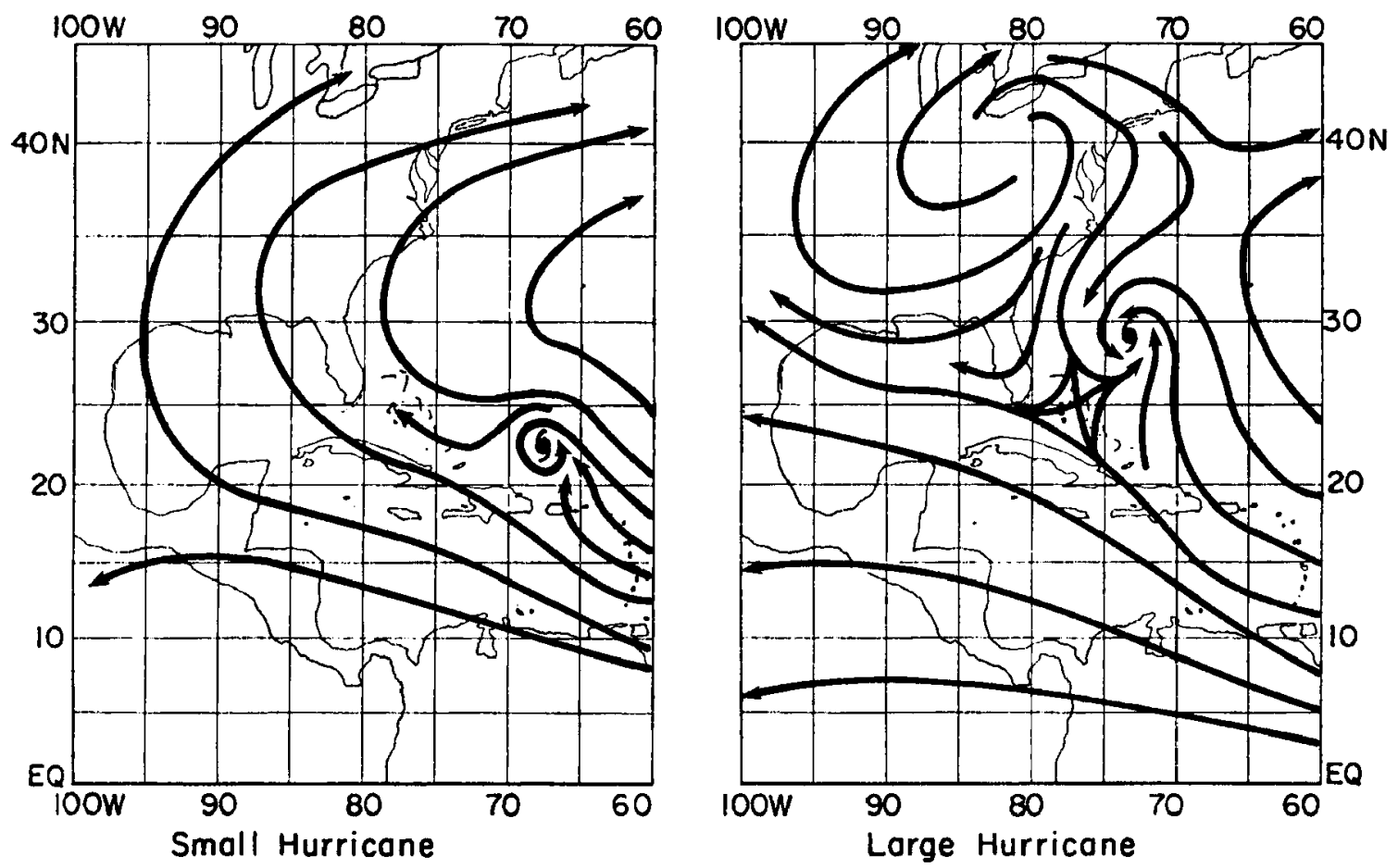

Fig. 48. Examples of environmental lower-tropospheric flows associated with a small cyclone (left) and a large or growing cyclone (right). 


\section{SUMMARY AND CONCLUSIONS}

This paper introduces the relatively unexplored topic of tropical cyclone size. A specification of the structure of a hurricane vortex is presented in terms of three parameters: intensity, size and strength. Size is estimated using the radius of outer closed isobar and a climatology of tropical cyclone size in the northwest Pacific and north Atlantic basins is presented. It is shown that Pacific cyclones are significantly larger, and that seasonal and geographical variability in tropical cyclone size occurs in both basins.

A first look at the structure of large and small tropical cyclones is obtained by compositing rawinsonde observations. Comparisons between large and small cyclones and intense and weak cyclones show the importance of size differences - large systems have lower-tropospheric winds at radii of $4^{\circ}-6^{\circ}$ latitude almost double those of small systems. The area involved in these wind differences is quite large. The effects of variations in intensity are confined to a much smaller area near the cyclone center.

Some of the dynamical implications of these structural differences are examined by computing the angular momentum and surface stresses associated with composite cyclones. When averaged over an area of $6^{\circ}$ latitude radius or more, angular momentum and surface losses are related more to size than intensity. An estimate of the horizontal flux of angular momentum required to produce observed rates of cyclone growth 
shows it to be several times larger than the flux needed to replace surface losses. Large cyclones occur as a result of formation in a highly cyclonic environment such as a monsoon trough, or by growth. Growth is hypothesized to result from enhanced momentum fluxes into the cyclone, most often as it moves poleward between a subtropical anticyclone and a polar outbreak anticyclone. Some specific findings of this paper are:

1) The definition of cyclone size based on the radius of outer closed isobar is a physically meaningful one, and the difference in lower tropospheric winds between large and smal1 cyclones is statistically significant.

2) Pacific cyclones are significantly larger than Atlantic cyclones.

3) Large cyclone frequency in both basins reaches a minimum in midsummer (July or August) and a maximum in October.

4) Large cyclones tend to occur in the vicinity of the monsoon trough (Pacific) and in the subtropics in autumn (Atlantic and Pacific).

5) Differences in tangential winds between tropical cyclones of different intensities are mainly confined to radii inside $2^{\circ}$.

6) Absolute angular momentum and surface losses of angular momentum are related more strongly to size than intensity when integrated over radii of $6^{\circ}$ or more.

7) Characteristic areas of large cyclone occurrence in the subtropics can be qualitatively explained by increased horizontal fluxes of angular momentum associated with cold surges impinging on the cyclones from the northwest. 
Much additional work remains to be done, as tropical cyclone growth, motion and intensification are coupled by means of vortexenvironment interactions. Of particular interest is the relationship between cyclone size, strength and intensity - growth seems explainable as a direct result of increased angular momentum fluxes into the cyclone, while strength and intensity changes are controlled by other factors as wel1. A 1ater research paper will attempt to address intensification directly. Additional work planned on tropical cyclone size will be efforts to develop information of operational use, such as a pressure-wind relationship including the effects of size, detailed statistics of the radius of gales around cyclones of different sizes, and a survey of the effect of cyclone size on hurricane surge and rainfa11. Efforts will also be made to determine whether the increased momentum fluxes can be consistently related to growth as has been hypothesized, and in exactly what ways the increased fluxes occur. 


\section{ACKNOWLEDGEMENTS}

This work represents the culmination of the labors, support and hopes of many. I am especially indebted to my advisor, Professor William M. Gray, for the continued opportunity and encouragement to work on this and related topics. Thanks go to: Professor Gray and members of his project, Greg Holland, Johnny Chan, Jianmin Xu, Clifford Matsumoto, and Cheng-Shang Lee for many fine discussions and words of advice; Janet Gray for keypunching the Atlantic size data set; Norine Haley for data reduction and plotting; Cindy Schrandt and Susan Cavender for typing; Edwin Buzze11 and many former students for rawinsonde data sets used in compositing; and to Barbara Brumit for typing and keping the whole show going.

This work was accomplished while the author was a National Science Foundation Graduate Fellow. Recommendation from the National Hurricane Center was instrumental in the obtaining this fellowship.

This research has been financially supported by NOAA Grant No. NA81RAD00005 and Grant No. NA81RAH00001 and NSF Grant No. ATM-7923591 Above a11, thanks to my parents for believing. 


\section{REFERENCES}

Arakawa, H., 1950: Mame-taifu or midget typhoon. Geophys. Mag., 23, 463-474.

Atkinson, G. D., 1971: Forecaster's guide to tropical meteorology. AWS Tech. Rept. 240, US Air Force.

Atkinson, G. D. and C. R. Holliday, 1977: Tropical cyclone minimum sea level pressure/maximum sustained wind relationship for the western north Pacific. Mon. Wea. Rev., 105, 421-427.

Australian Bureau of Meteorology, 1977: Report on cyclone Tracy, December, 1974. Australian Government Publishing Service, Canberra, 82 pp.

B1ack, P. G. and R. A. Anthes, 1971: On the asymmetric structure of the tropical cyclone outflow 1ayer. I. Atmos. Sci., 28, 1348-1366.

Brand, S., 1972: Very large and very sma11 typhoons of the western north Pacific Ocean. I. Meteor. Soc. Japan, 50, 332-341.

Chan, J. C. L. and W. M. Gray, 1982: Tropical cyclone motion and surrounding parameter relationships. Submitted to Mon. Wea. Rev.

Chan, J. C. L., W. M. Gray and S. Q. Kidder, 1980: Forecasting tropical cyclone turning motion from surrounding wind and temperature fields. Mon. Wea. Rev., 108, 778-792.

Ding, Y. H, and E. R. Reiter, 1980: A preliminary study of the variability in the frequency of typhoon formation over the west Pacific Ocean. Environmental Research Paper 22, Co1o. State Univ., Fort Collins, Co, 21 pp.

Dunn, G. E. and B. I. Miller, 1960: Atlantic Hurricanes. Louisiana State Univ. Press, Baton Rouge, LA, 326 pp.

Dunnavan, G. M. and J. W. Diercks, 1980: An analysis of super typhoon Tip (October 1979). Mon. Wea. Rev., 108, 1915-1923.

Dvorak, V. F., 1975: Tropica1 cyclone intensity analysis and forecasting from satellie imagery. Mon. Wea. Rev., 103, 420-430.

Frank, W. M., 1977: The structure and energetics of the tropical cyclone, Part I: Storm structure. Mon. Wea. Rev., 105, 1119-1135.

Frank, W. M. and W. M. Gray, 1980: Radius and frequency of $15 \mathrm{~m} \mathrm{~s}^{-1}$ ( 30 kt) winds around tropical cyclones. I. Appl. Meteor., 19, 219-223. 


\section{REFERENCES (cont'd)}

Garratt, J. R., 1977: Review of drag coefficients over oceans and continents. Mon. Wea. Rev., 105, 915-929.

Gray, W. M., 1979: Hurricanes: their formation, structure and like1y role in the tropical circulation. Supplement to Meteorology Over the Tropical Oceans. Published by RMS, James Glaisher House, Grenville P1ace, Brackne11, Berkshire, R6 12 1BX, D. B. Shaw, Ed., 155-218.

Gray, W. M., E. Buzze11, G. Burton and Other Project Personne1, 1982: Tropical cyclone and related meteorological data sets available at CSU and their utilization. Dept. of Atmos. Sci. Paper, Colo. State Univ., Ft. Collins, CO, $186 \mathrm{pp}$.

George, J. E. and W. M. Gray, 1977: Tropical cyclone recurvature and non-recurvature as related to surrounding wind-height fields. J. App1. Meteox., 16, 34-42.

Hol1and, G. J., 1982: Angular momentum transports in tropical cyclones. Accepted for publication by Quart. I. Roy. Meteor. Soc.

Hughes, L. A., 1952: On the low-leve1 wind structure of tropical storms. I. Meteor., 9, 422-428.

Jarvinen, B. R. and E. L. Caso, 1978: A tropical cyclone data tape for the north Atlantic basin, 1886-1977: contents, 1 imitations and uses. NOAA Tech. Memo., NWS NHC-6, 19 pp.

Lee, C. S., 1982: Cumnlus momentum transports in tropical cyclones. Dept. of Atmos. Sci. Paper No. 341, Co1o. State Univ., Ft. Collins, Co, $78 \mathrm{pp}$.

Love, G., 1982: The role of the general circulation in western Pacific tropical cyclone genesis. Dept. of Atmos. Sci. Paper No, 340, Co10. State Univ., Ft. Co11ins, CO, $215 \mathrm{pp}$.

McBride, J. L. and R. Zehr, 1981: Observational studies of tropica1 cyclone formation, Part II: Comparison of non-developing versus developing systems. I. Atmos. Sci., 1132-1151.

Moste11er, F. and R. E. K. Rourke, 1973: Sturdy statistics. AddisonWesley Pub1ishing Co., Reading, MA, 395 pp.

Pfeffer, R. L., 1958: Concerning the mechanics of hurricanes. I. Meteor., 15, 113-120.

Rieh1, H., 1979: C1imate and weather in the tropics. Academic Press, London, $611 \mathrm{pp}$.

Rieh1, H, and J. S. Ma1kus, 1960: On the dynamics and energy transformations in steady-state hurricanes. Tellus, 12, 1-20. 
REFERENCES (cont'd)

Shea, D. J. and W. M. Gray, 1973: The hurricane's inner core regions, I: Symmetric and a symmetric structure. I. Atmos. Sci., 30, 15441564 .

Walpole, R. E. and R. H. Myers, 1978: Probability and statistics for engineers and scientists. Macmillan, Collier, New York, 580 pp.

Zipser, E. J., 1964: On the thermal structure of developing tropica1 cyclones. NHRP Report 67, National Hurricane Research Project, Miami, FL, 23 pp. 


\section{APPENDIX}

The mean azimuthal mean tangential winds for the composite tropical cyclones described in Chapter 4 are shown below.

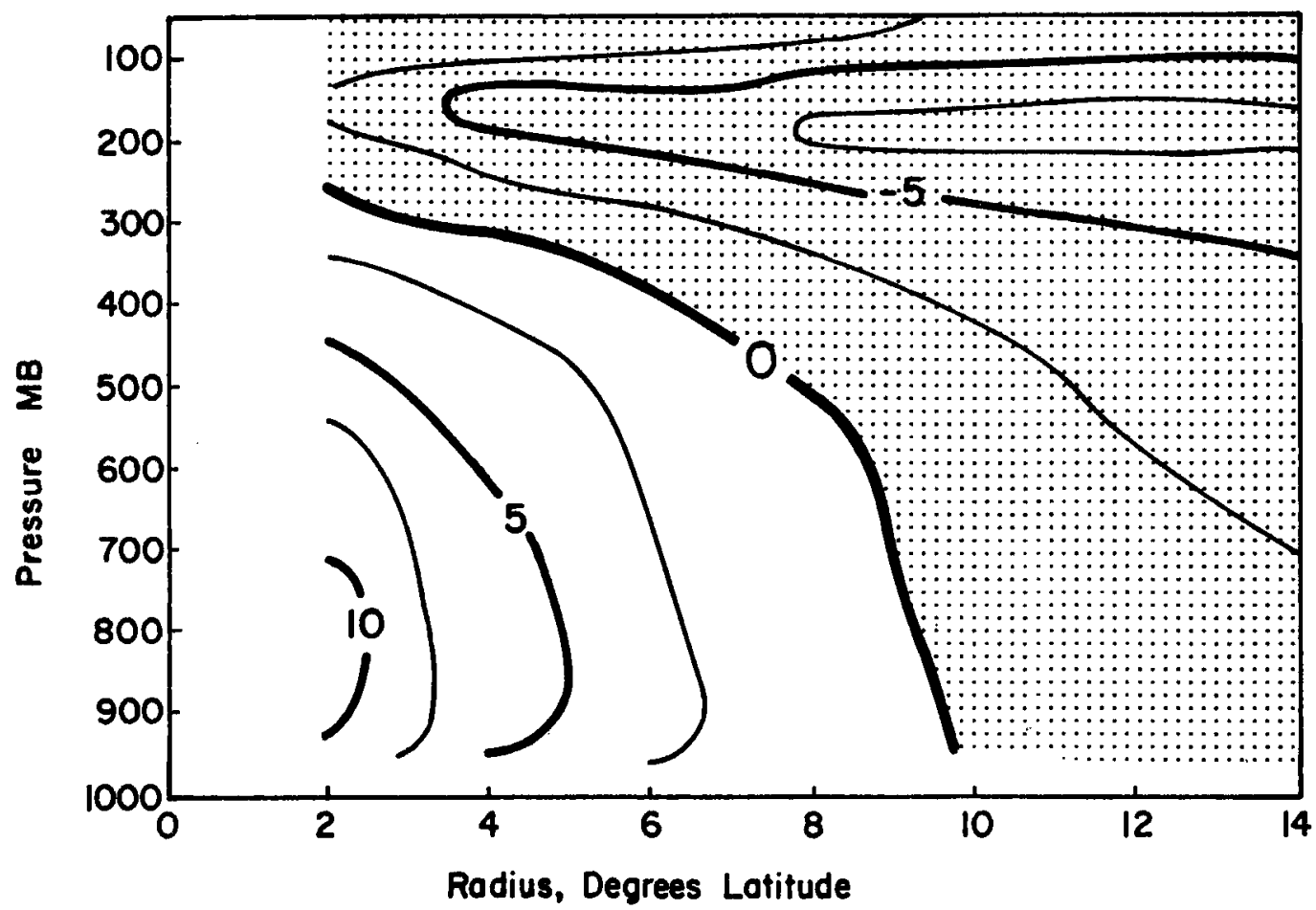

Fig. 49. Azimuthal mean tangential wind for the composite Atlantic sma11/medium tropical storm. 


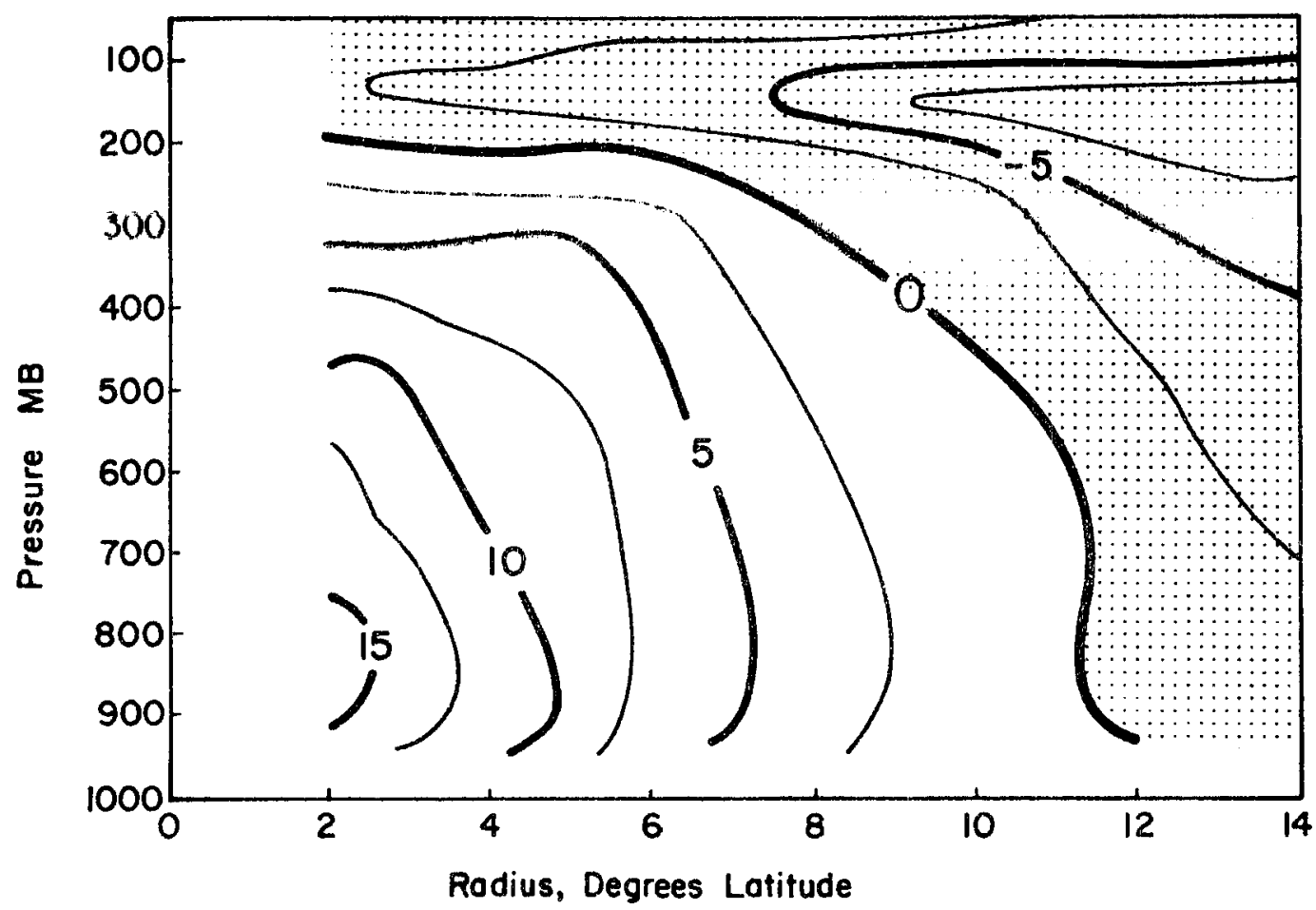

Fig. 50. Same as Fig. 49 except for the Atlantic large tropical storm.

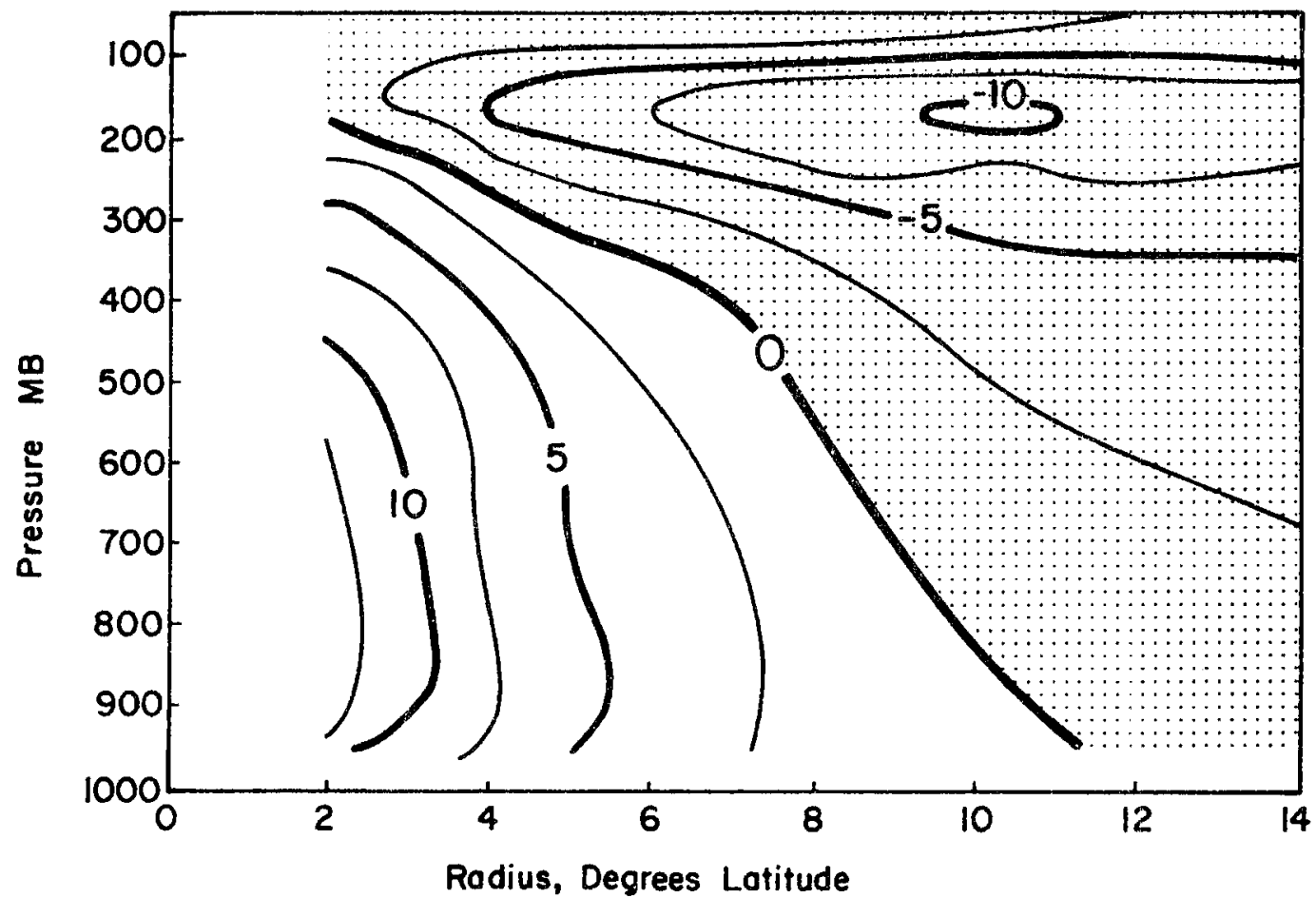

Fig. 51. Same as Fig. 49 except for the Atlantic sma11/medium hurricane. 


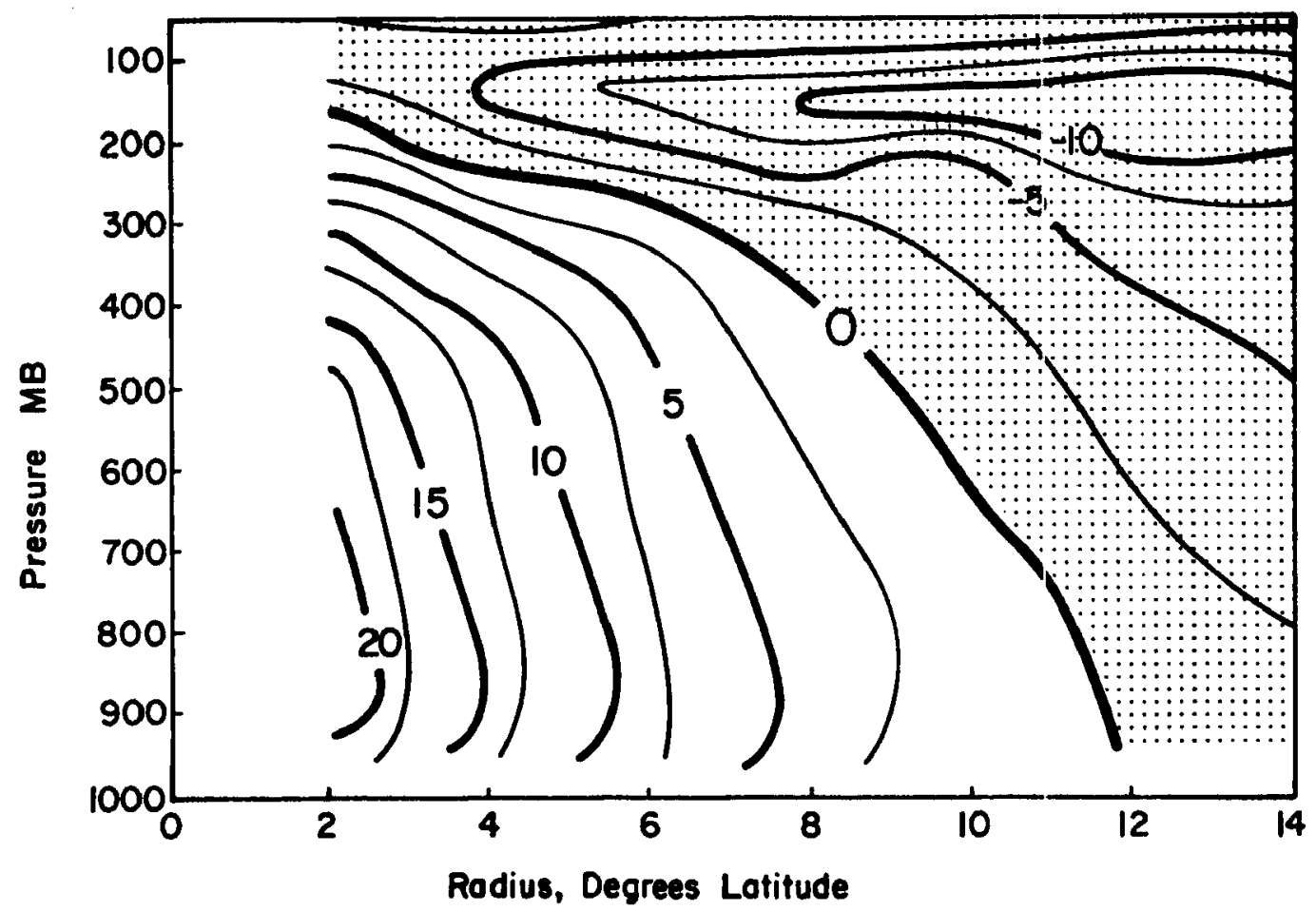

Fig. 52. Same as Fig. 49 except for the Atlantic large hurricane north of $25 \mathrm{~N}$.

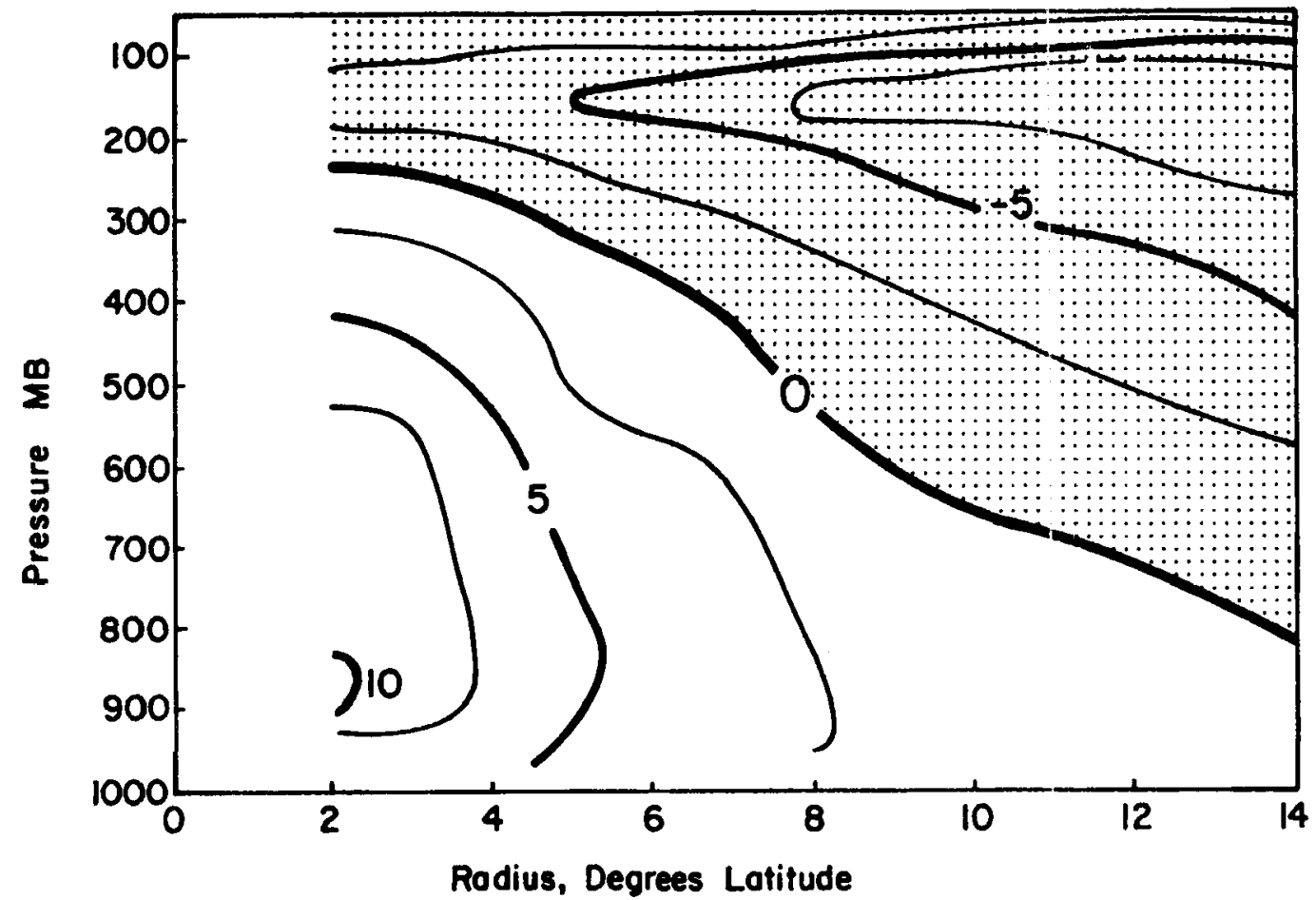

Fig. 53. Same as Fig. 49 except for the Pacific sma11 tropical storm. 


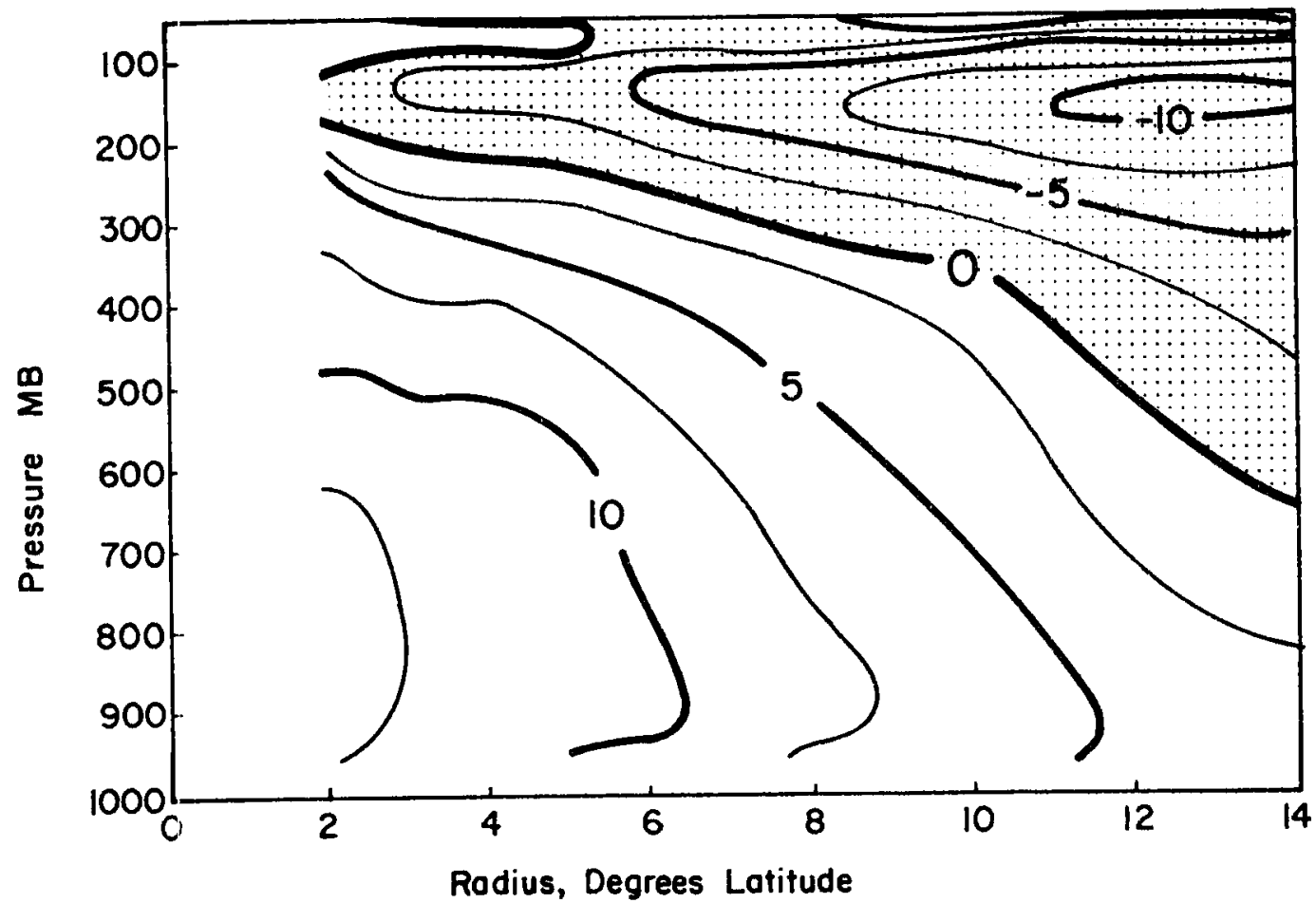

Fig. 54. Same as Fig. 49 except for the Pacific large tropical storm.

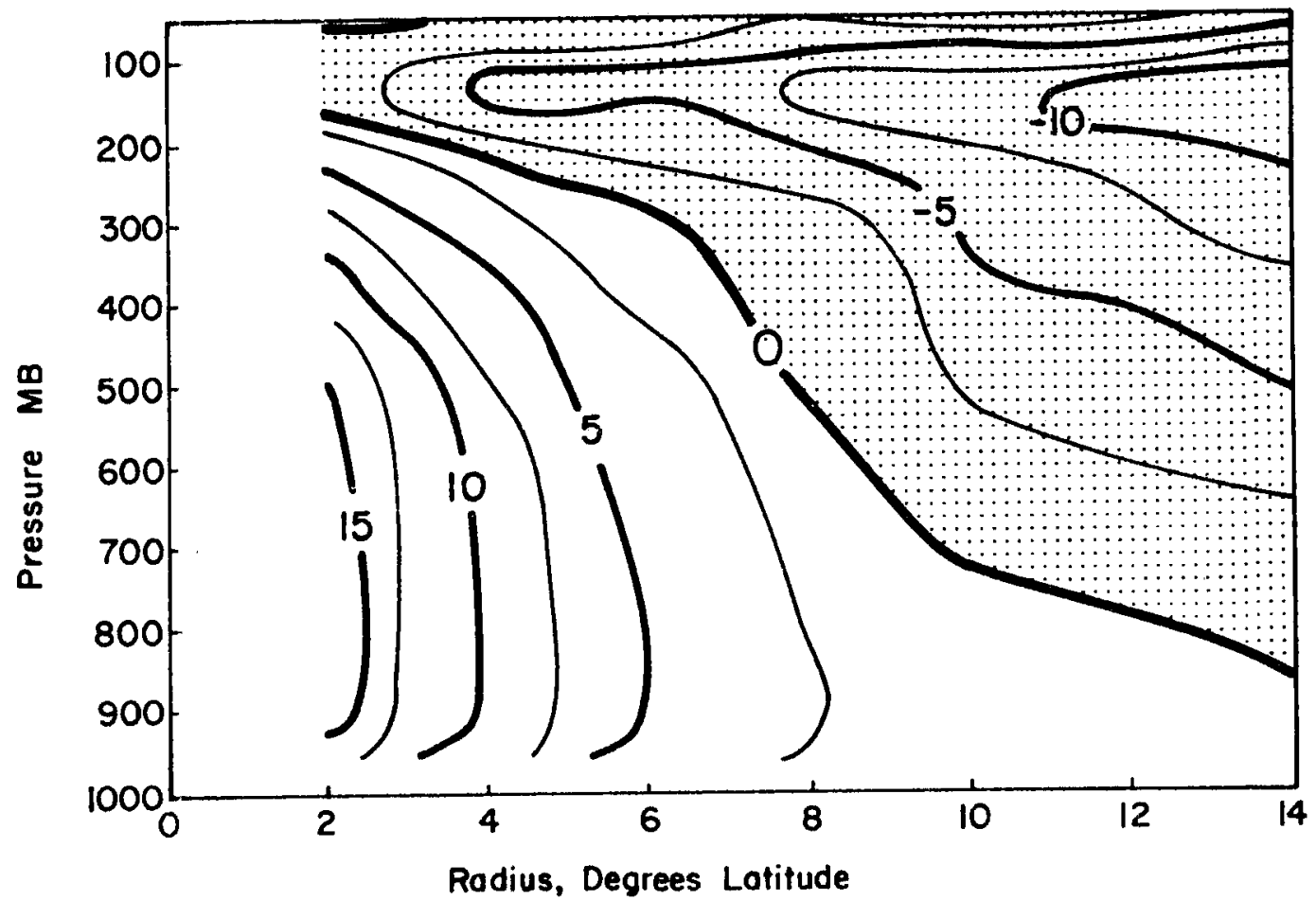

Fig. 55. Sane as Fig. 49 except for the Pacific small typhoon. 


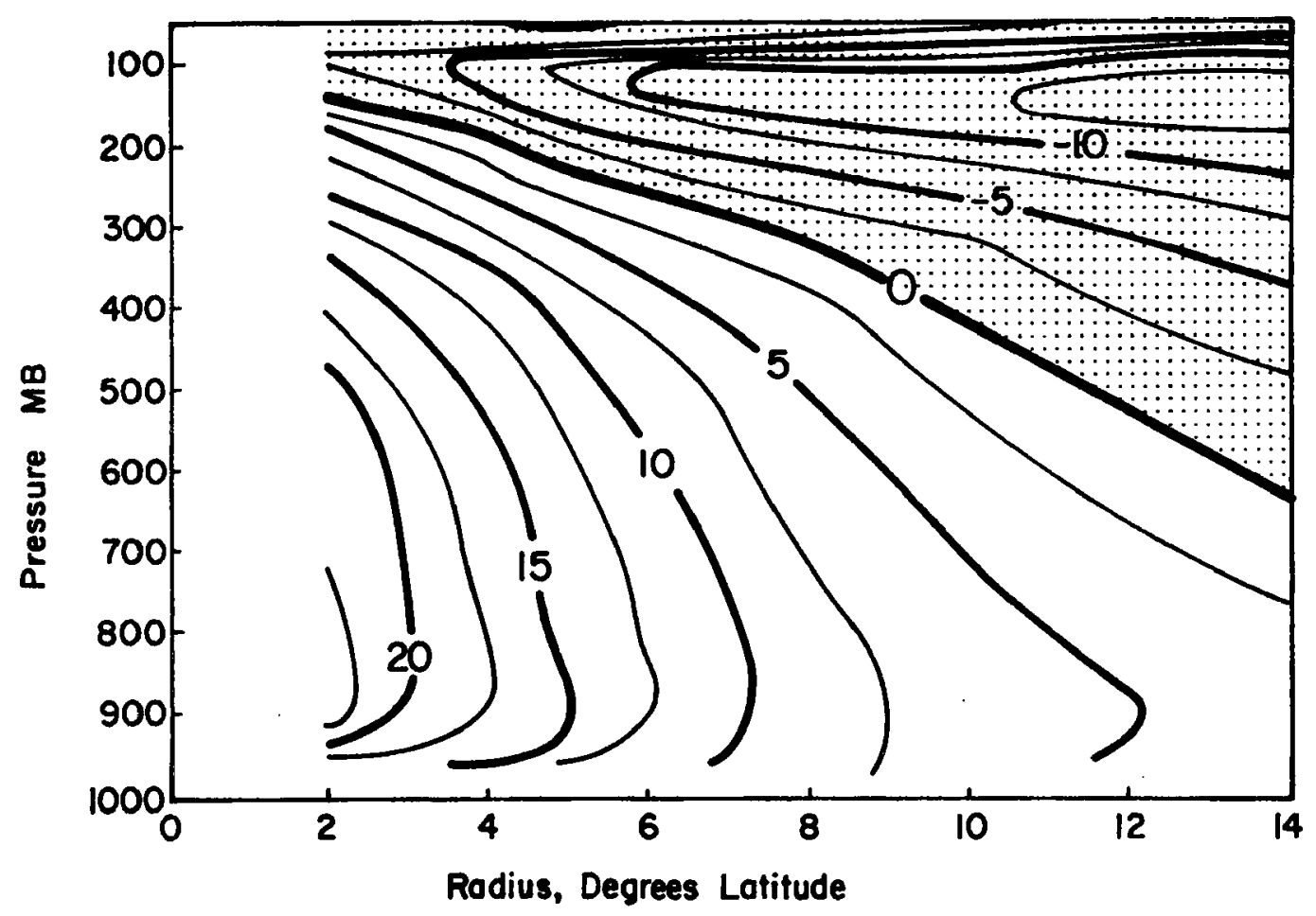

Fig. 56. Same as Fig. 49 except for the Pacific large typhoon. 
W. M. GRAY'S FEDERALLY SUPPORTED RESEARCH PROJECT REPORTS SINCE 1967

CSU Dept. of Atmos. Sci. Report No.

104

114

116

124

Unnumbered

Unnumbered

140

161

179

182

\section{Report Tit1e, Author, Date, Agency Support}

The Mutual Variation of Wind, Shear and Baroc1inicity in the Cumulus Convective Atmosphere of the Hurricane (69 pp.). W. M. Gray. February 1967. NSF Support.

Global View of the Origin of Tropical Distuxbances and Storms (105 pp.). W. M. Gray. October 1967. NSF Support.

A Statistical Study of the Frictional Wind Veering in the Planetary Boundary Layer (57 pp.). B. Mendenha11. December 1967. NSF and ESSA Support.

Investigation of the Importance of Cumulus Convection and Ventilation in Early Tropical Storm Development ( 88 pp.). R. Lopez. June 1968. ESSA Sate11ite Lab. Support.

Role of Angular Momentum Transports in Tropical Storm Dissipation over Tropical 0ceans ( 46 pp.). R. F. Wachtmann. December 1968. NSF and ESSA Support.

Monthly C1imatological Wind Fields Associated with Tropica1 Storm Genesis in the West Indies (34 pp.). J. W. Sartor. December 1968. NSF Support.

Characteristics of the Tornado Environment as Deduced from Proximity Soundings (55 pp.). T. G. Wills. June 1969. NOAA and NSF Support.

Statistical Analysis of Trade Wind Cloud C1usters in the Western North Pacific ( $80 \mathrm{pp}$.$) . K. Williams. June 1970$. ESSA Sate11ite Lab. Support.

A C1imatology of Tropica1 Cyclones and Disturbances of the Western Pacific with a Suggested Theory for Their Genesis/ Maintenance (225 pp.). W. M. Gray. NAVWEARSCHFAC Tech. Paper No. 19-70. November 1970. (Available from US Navy, Monterey, CA). US Navy Support.

A diagnostic Study of the P1anetary Boundary Layer over the Oceans ( 95 pp.). W. M. Gray. February 1972. Navy and NSF Support.

The Structure and Dynamics of the Hurricane's Inner Core Area (105 pp.). D. J. Shea. April 1972. NOAA and NSF Support. 
CSU Dept. of Atmos. Sci. Report No.

188

189

190

195

196

199

200

212

219

224

225

234

Report Title, Author, Date, Agency Support

Cumulus Convection and Larger-scale Circulations, Part I:

A Parametric Model of Cumulus Convection (100 pp.).

R. E. Lopez. June 1972. NSF Support.

Cumulus Convection and Larger-scale Circulations, Part II:

Cumulus and Meso-scale Interactions (63 pp.). R. E. Lopez. June 1972. NSF Support.

Cumulus Convection and Larger-scale Circulations, Part III: Broadscale and Meso-scale Considerations ( 80 pp.). W. M. Gray. Ju1y 1972. NOAA-NESS Support.

Characteristics of Carbon Black Dust as a Tropospheric Heat Source for Weather Modification (55 pp.). W. M. Frank. January 1973. NSF Support.

Feasibility of Beneficial Hurricane Modification by Carbon Black Seeding (130 pp.). W. M. Gray. April 1973. NOAA Support.

Variability of Planetary Boundary Layer Winds (157 pp.). L. R. Hoxit. May 1973. NSF Support.

Hurricane Spawned Tornadoes (57 pp.). D. J. Nov1an. May 1973. NOAA and NSF Support.

A Study of Tornado Proximity Data and an Observationally Derived Model of Tornado Genesis (101 pp.). R. Maddox. November 1973. NOAA Support.

Analysis of Satellite Observed Tropical Cloud Clusters (91 pp.). E. Ruprecht and W. M. Gray. May 1974. NOAA/ NESS Support.

Precipitation Characteristics in the Northeast Brazil Dry Region ( 56 pp.). R. P. L. Ramos. May 1974. NSF Support.

Weather Modification through Carbon Dust Absorption of Solar Energy (190 pp.). W. M. Gray, W. M. Frank, M. L. Corrin, and C. A. Stokes. July 1974.

Tropical Cyclone Genesis (121 pp.). W. M. Gray. March 1975. NSF Support. 
CSU Dept. of Atmos. Sci. Report No.

241

243

257

259

Unnumbered

262

\section{Report Title, Author, Date, Agency Support}

Tropical Cyclone Genesis in the Western North Pacific (66 pp.). W. H. Gray. March 1975. US Navy Environmenta1 Prediction Research Facility Report. Tech. Paper No. 16-75. (Available from the US Navy, Monterey, CA). Navy Support.

Tropical Cyclone Motion and Surrounding Parameter Re1ationships (105 pp.). J. E. George. December 1975. NOAA Support.

Diurnal Variation of Oceanic Deep Cumulus Convection. Paper I: Observationa1 Evidence, Paper II: Physica1 Hypothes is (106 pp.). R. W. Jacobson, Jr. and W. M. Gray. February 1976. NOAA-NESS Support.

Data Summary of NOAA's Hurricanes Inner-Core Radial Leg F1ight Penetrations 1957-1967, and 1969 (245 pp.). W. U. Gray and D. J. Shea. October 1976. NSF and NOAA Support.

The Structure and Energetics of the Tropical Cyclone (180 pp.). W. M. Frank. October 1976. NOAA-NHEML, NOAA-NESS and NSF Support.

Typhoon Genesis and Pre-typhoon Cloud Clusters (79 pp.). R. M. Zehr. November 1976. NSF Support.

Severe Thunderstorm Wind Gusts ( 81 pp.). G. W. Wa1ters. December 1976. NSF Support.

Diurnal Variation of the Tropospheric Energy Budget (141 pp.). G. S. Fo1tz. November 1976. NSF Support.

Comparison of Developing and Non-developing Tropical Disturbances ( $81 \mathrm{pp.})$. S. L. Erickson. Ju1y 1977. US Army Support.

Tropical Cyclone Research by Data Compositing (79 pp.). W. M. Gray and W. M. Frank. Ju1y 1977. US Navy Environmenta1 Prediction Research Facility Report. Tech. Paper No. 77-01. (Available from the US Navy, Monterey, CA.). Navy Support.

Tropical Cyclone Clond and Intensity Relationships (154 pp.). C. P. Arnold. November 1977. US Army and NHEML Support.

Diagnostic Analyses of the GATE A/B-scale Area at Individual Time Periods (102 pp。). W. M. Frank. November 1978. NSF Support. 
CSU Dept. of Atmos. Sci. Report No.

Report Tit1e, Author, Date, Agency Support

Diurnal Variability in the GATE Region ( $80 \mathrm{pp.})$. J. M. Dewart. November 1978. NSF Support.

Mass Divergence in Tropical Weather Systems, Paper I: Diurnal Variation; Paper II: Large-scale Controls on Convection (109 pp.). J. L. McBride and W. M. Gray. November 1978. NOAA-NHEML Support.

New Results of Tropical Cyclone Research from Observational Analysis (108 pp.). W. M. Gray and W. M. Frank. June 1978. US Navy Environmental Prediction Research Facility Report. Tech. Paper No. 78-01. (Available from the US Navy, Monterey, CA). Navy Support.

Convection Induced Temperature Change in GATE (128 pp.). P. G. Grube. February 1979. NSF Support.

Observational Analysis of Tropical Cyclone Formation (230 pp.). J. L. McBride. April 1979. NOAA-NHEML, NSF and NEPRF Support.

Tropical Cyclone Intensity Change - A Quantitative Forecasting Scheme. K. M. Dropco. May 1981. NOAA Support.

The Role of the General Circulation in Tropical Cyclone Genesis (230 pp.). G. Love. Apri1 1982. NSF Support.

Cumulus Momentum Transports in Tropical Cyclones (78 pp.). C. S. Lee. May 1982. ONR Support.

Tropical Cyclone Movement and Surrounding F1ow Relationships (68 pp.). J. C. L. Chan and W. M. Gray. May 1982. ONR Support.

Environmental Circulations Associated with Tropical Cyclones Experiencing Fast, Slow and Looping Motions (273 pp.). J. Xu and W. M. Gray. May 1982. ONR Support.

Tropical Cyclone Motion: Environmental Interaction Plus a Beta Effect (47 pp.). G. J. Holland. May 1982. ONR Support.

Tropica1 Cyclone and Related Meteorological Data Sets Available at CSU and Their Utilization (186 pp.). W. M. Gray, E. Buzze11, G. Burton and 0ther Project Personnel. February 1982. NSF, ONR, NOAA, and NEPRF Support. 